\title{
Duality between unprovability and provability in forward proof-search for Intuitionistic Propositional Logic
}

\author{
Camillo Fiorentini $^{1}$, Mauro Ferrari ${ }^{2}$ \\ 1 DI, Univ. degli Studi di Milano, Via Comelico, 39, 20135 Milano, Italy \\ 2 DiSTA, Univ. degli Studi dell'Insubria, Via Mazzini, 5, 21100, Varese, Italy
}

\begin{abstract}
The inverse method is a saturation based theorem proving technique; it relies on a forward proof-search strategy and can be applied to cut-free calculi enjoying the subformula property. Here we apply this method to derive the unprovability of a goal formula $G$ in Intuitionistic Propositional Logic. To this aim we design a forward calculus $\mathbf{F R J}(G)$ for Intuitionistic unprovability which is prone to constructively ascertain the unprovability of a formula $G$ by providing a concise countermodel for it; in particular we prove that the generated countermodels have minimal height. Moreover, we clarify the role of the saturated database obtained as result of a failed proof-search in $\mathbf{F R J}(G)$ by showing how to extract from such a database a derivation witnessing the Intuitionistic validity of the goal.
\end{abstract}

\section{Introduction}

The inverse method, introduced in the 1960s by Maslov [17, is a saturation based theorem proving technique closely related to (hyper)resolution [6]; it relies on a forward proof-search strategy and can be applied to cut-free calculi enjoying the subformula property. Given a goal, a set of instances of the rules of the calculus at hand is selected; such specialized rules are repeatedly applied in the forward direction, starting from the axioms (i.e., the rules without premises). Proof-search terminates if either the goal is obtained or the database of proved facts saturates (no new fact can be added). As pointed out by Vladimir Lifschitz [16], "the role of the inverse method in the Soviet work on proof procedures for predicate logic can be compared to the role of resolution method in theorem proving projects in the West". But, he regrets, "for a number of reasons, this work has not been duly appreciated outside a small circle of Maslov's associates". The method has been popularized by Degtyarev and Voronkov [6], who provide the general recipe to design forward calculi, with applications to Classical Predicate Logic and some non-classical logics. Further extensions can be found in 2/7]15. A significant investigation is presented in [4], where focused calculi and polarization of formulas are exploited to reduce the search space in forward proof-search for Intuitionistic Logic. These techniques are at the heart of the design of the prover Imogen [18. 
In all the mentioned papers, the inverse method has been exploited to prove the validity of a goal in a specific logic. Here we follow the dual approach, namely: we design a forward calculus to derive the unprovability of a goal formula in Intuitionistic Propositional Logic (IPL). Our motivation is twofold. Firstly, we aim to define a calculus which is prone to constructively ascertain the unprovability of a formula by providing a concise countermodel for it. The second motivation is to clarify the role of the saturated database obtained as result of a failed proofsearch. In the case of the usual forward calculi for Intuitionistic provability, if proof-search fails, a saturated database is generated which "may be considered a kind of countermodel for the goal sequent" [18. However, as far as we know, no method has been proposed to effectively extract it. Actually, the main problem comes from the high level of non-determinism involved in the construction of countermodels. Here, assuming the dual approach, the saturated database generated by a failed proof-search can be considered as "a kind of proof of the goal"; we give evidence of this by showing how to extract from such a database a derivation witnessing the Intuitionistic validity of the goal.

Our different perspectives requires a deep adjustment of the inverse method itself. Sequents $\Gamma \vdash C$ of standard forward calculi encode the fact that the right formula $C$ is provable from the set of left formulas $\Gamma$ in the understood logic. In our approach, a sequent $\Gamma \Rightarrow C$ signifies the unprovability of $C$ from $\Gamma$ in IPL. From a semantic viewpoint, this means that, in some world of a Kripke model, all the formulas in $\Gamma$ are forced and $C$ is not forced. In standard forward reasoning, axioms have the form $p \vdash p$, where $p$ is a propositional variable. In our approach, axioms have the form $\Gamma^{\text {At }} \Rightarrow p$ or $\Gamma^{\text {At }} \Rightarrow \perp$, where $\Gamma^{\text {At }}$ is a set of propositional variables and $p$ is a propositional variable not belonging to $\Gamma^{\text {At }}$. Rules must preserve (top-down) unprovability. Examples of sound rules for unprovability are:

$$
\frac{\Gamma \Rightarrow A}{\Gamma \Rightarrow A \wedge B} R \wedge \quad \frac{A, \Gamma \Rightarrow C}{A \vee B, \Gamma \Rightarrow C} L \vee
$$

The former rule states that if $A$ is not provable from $\Gamma$, then $A \wedge B$ is not provable from $\Gamma$. The latter corresponds to the contrapositive of Inversion Principle for left $\vee$ : if $C$ is not provable from $\{A\} \cup \Gamma$, then $C$ is not provable from $\{A \vee B\} \cup \Gamma$. The tricky point is how to cope with rules having more than one premise. In direct forward calculi, left formulas must be gathered. For instance, the rule for right $\wedge$

$$
\frac{\Gamma_{1} \vdash A \quad \Gamma_{2} \vdash B}{\Gamma_{1}, \Gamma_{2} \vdash A \wedge B} R \wedge
$$

encodes the property that if $A$ is provable from $\Gamma_{1}$ and $B$ is provable from $\Gamma_{2}$, then $A \wedge B$ is provable from $\Gamma_{1} \cup \Gamma_{2}$. Apparently, in the forward refutation calculus we should follow the dual approach and intersect left formulas. Thus, the rule $R \vee$ should be

$$
\frac{\Gamma_{1} \Rightarrow A \quad \Gamma_{2} \Rightarrow B}{\Gamma_{1} \cap \Gamma_{2} \Rightarrow A \vee B} R \vee
$$

to be interpreted as "if $A$ is not provable from $\Gamma_{1}$ and $B$ is not provable from $\Gamma_{2}$, then $A \vee B$ is not provable from $\Gamma_{1} \cap \Gamma_{2}$ ". But the alleged rule $R \vee$ does not 
preserve unprovability, as shown by this trivial counterexample:

$$
\frac{q_{2}, p, H \Rightarrow q_{1} \quad q_{1}, p, H \Rightarrow q_{2}}{p, H \Rightarrow q_{1} \vee q_{2}} R \vee \quad H=p \supset q_{1} \vee q_{2}
$$

Here $q_{1}$ is not provable from $\Gamma_{1}=\left\{q_{2}, p, H\right\}$ and $q_{2}$ is not provable from $\Gamma_{2}=$ $\left\{q_{1}, p, H\right\}$, but the right formula of the conclusion $q_{1} \vee q_{2}$ is provable from $\Gamma_{1} \cap$ $\Gamma_{2}=\{p, H\}$. The drawback is that the conclusion cannot retain both $p$ and $H$ in left. To get a sound rule, we have to select a suitable subset $\Gamma_{0}$ of $\Gamma_{1} \cap \Gamma_{2}$ : the possible choices are $\Gamma_{0}=\{p\}$ or $\Gamma_{0}=\{H\}$ or $\Gamma_{0}=\emptyset$. Thus, we need a more clever strategy to join sequents and to treat left formulas in multi-premise rules. In addition to the sequents mentioned so far, we call regular sequents, we introduce irregular sequents of the kind $\Sigma ; \Theta \rightarrow C$, where the left formulas are partitioned into two sets $\Sigma$ and $\Theta$; in forward proof-search, formulas in the sets $\Sigma$ must be kept as much as possible.

The definition of the rules of a forward calculus depends on the formula to be proved (the goal formula). The calculus we define is parametrized by the goal formula $G$ (where the goal is to prove that $G$ is not valid in IPL). We call the related calculus FRJ $(G)$ (Forward Refutation calculus for IPL parametrized by $G$ ); formulas occurring in the sequents of $\mathbf{F R J}(G)$ are suitable subformulas of $G$. The rules of the calculus are shown in Fig. 1 and discussed in Sect. 3 . We point out that, differently from standard sequent calculi, left-hand sides of sequents only host propositional variables and implicative formulas $A \supset B$; moreover $\mathbf{F R J}(G)$ only supplies right rules. In Sec. 4 we define a forward proofsearch procedure to build an $\mathbf{F R J}(G)$-derivation of a goal formula $G$, namely an FRJ $(G)$-derivation of a regular sequent of the form $\Gamma \Rightarrow G$. This is a standard saturation procedure where the provable sequents of $\mathbf{F R J}(G)$ are collected stepby-step in a database $\mathrm{D}_{G}$. Initially $\mathrm{D}_{G}$ contains all the axioms of $\mathbf{F R J}(G)$; then a loop is entered where the rules of the calculus are repeatedly applied (in the forward direction) to the sequents in $\mathrm{D}_{G}$. The loop ends when either $G$ is proved or no new sequent can be added to $\mathrm{D}_{G}$; since the number of sequents of $\mathbf{F R J}(G)$ is bounded, the process eventually ends. To avoid redundancies and maintain $\mathrm{D}_{G}$ compact, we introduce a subsumption relation between sequents; for instance, if at some step $\sigma$ is proved and $\sigma$ is subsumed by a sequent already in $\mathrm{D}_{G}$, then $\sigma$ is discarded and not added to $\mathrm{D}_{G}$ (forward subsumption).

The rules of $\mathbf{F R J}(G)$ are inspired by Kripke semantics. In Sec. 3.1 we show that, from a derivation of $G$, we can extract a countermodel for $G$, namely a Kripke model such that, at its root, the formula $G$ is not forced, witnessing that $G$ is not valid in IPL [3]. Actually, there is a close correspondence between a derivation and the related Kripke model. Thus, our forward proof-search procedure can be understood as a top-down method to build a countermodel for $G$, starting from the final worlds down to the root. This original approach is dual to the standard one, where countermodels are built bottom-up, mimicking the backward application of rules (see.e.g., 1 18 9 10]13[19|20]). This different viewpoint has a significant impact in the outcome. Indeed, the countermodels generated by a backward procedure are always trees, which might contain some redundancies. Instead, forward methods are prone to re-use sequents and to not replicate 
them; thus the generated models do not contain duplications and are in general very concise (see the compact models in Figs. 6 and 11). In Sec. 3 we show that FRJ $(G)$-derivations have height quadratic in $|G|$ (the size of $G$ ). Moreover, if $G$ is not valid, the countermodel extracted from an $\mathbf{F R J}(G)$-derivation of $G$ has height at most $|G|$.

The relationship between a non valid formula $G$ and the height of the countermodel extracted from an $\mathbf{F R J}(G)$-derivation of $G$ is deeply investigated in Sec. 6. Here we show that, given a countermodel for $G$ of height $h$, we can build an $\mathbf{F R J}(G)$-derivation of $G$ having height at most $h$. By this fact, we conclude that, if $G$ is not valid in IPL, we can build an $\mathbf{F R J}(G)$-derivation of $G$ such that the height $h$ of the extracted countermodel is minimal (namely, there exists no countermodel for $G$ having height less than $h$ ). We can tweak the proof-search procedure so that, if $G$ is non valid, it yields an $\mathbf{F R J}(G)$-derivation of $G$ such that the extracted countermodel has minimal height.

If the formula $G$ is valid in IPL, proof-search for $G$ fails (indeed, no $\mathbf{F R J}(G)$ derivation of $G$ can be built) and we eventually get a saturated database $\mathrm{D}_{G}$ for $G$. This means that for every sequent $\sigma$ provable in $\mathbf{F R J}(G), \mathrm{D}_{G}$ contains a sequent $\sigma^{\prime}$ which subsumes $\sigma$; thus $\mathrm{D}_{G}$ is in some sense representative of all the sequents provable in $\mathbf{F R J}(G)$. We can exploit $\mathrm{D}_{G}$ to build a derivation of $G$ in a sequent calculus for IPL. To this aim, in Sec. 5 we introduce the sequent calculus $\mathbf{G b u}(G)$ (see Fig. 9), a variant of the well-known sequent calculus G3i 23. From a $\mathbf{G b u}(G)$-derivation of $G$, we can immediately obtain a G3i-derivation of $G$. Differently from G3i, backward proof-search in $\mathbf{G b u}(G)$ always terminate; indeed, we can define a weight function on sequents such that, after the backward application of a rule of $\mathbf{G b u}(G)$ to a sequent, the weight of the sequents decreases. Nonetheless, backward-proof search in $\mathbf{G b u}(G)$ might present several backtrack points, in correspondence of the applications of rules for left implication and right disjunction. The crucial point is that we can remove backtracking by querying the database $\mathrm{D}_{G}$ : in presence of multiple non-deterministic choices, we exploit $\mathrm{D}_{G}$ to select the right way so to successfully continue proof-search. Thus, we can consider $\mathrm{D}_{G}$ as a proof-certificate of the validity of $G$, in the sense that it contain enough information to reconstruct a derivation of $G$ in the sequent calculus G3i. If we eliminate from $\mathrm{D}_{G}$ all the redundancies (if $\sigma$ belongs to $\mathrm{D}_{G}$, then remove from $\mathrm{D}_{G}$ all the sequents subsumed by $\sigma$ ), then we get a saturated database $\mathrm{D}_{G}^{*}$ which is the minimum among the saturated databases of $G$, hence we can consider $\mathrm{D}_{G}^{*}$ as the canonical proof-certificate of the validity of $G$. To get the minimum saturated database, we have to enhance the proof-search procedure by implementing backward subsumption.

To evaluate the potential of our approach we have implemented $f r j$, a Java prototype of our proof-search procedure based on the JTabWb framework $11{ }^{3}$ frj implements term-indexing, forward and backward subsumption and it allows the user to generate the rendering of proofs and of the extracted countermodels.

\footnotetext{
${ }^{3} \mathrm{frj}$ is available at http://github.com/ferram/jtabwb_provers/.
} 


\section{Preliminaries}

We consider the propositional language $\mathcal{L}$ based on a denumerable set of propositional variables $\mathcal{V}$, the connectives $\wedge, \vee, \supset$ (as usual, $\wedge$ and $\vee$ bind stronger than $\supset$ ) and the logical constant $\perp ; \neg A$ is a shorthand for $A \supset \perp$. If $\odot$ is a logical connective, we call $\odot$-formula a formula with top-level connective $\odot$. By $\mathcal{V}^{\perp}$ we denote the set $\mathcal{V} \cup\{\perp\}$ and by $\mathcal{L}^{\supset}$ the set of $\supset$-formulas of $\mathcal{L}$. Capital Greek letters $\Gamma, \Sigma, \ldots$ denote sets of formulas; we use notations like $\Gamma^{\text {At }}$ and $\Gamma^{\supset}$ to mean that $\Gamma^{\text {At }} \subseteq \mathcal{V}$ and $\Gamma^{\supset} \subseteq \mathcal{L}^{\supset}$. Given a formula $G, \operatorname{Sf}(G)$ is the set of all subformulas of $G$ (including $G$ itself). $\operatorname{By} \operatorname{SL}(G)$ and $\operatorname{SR}(G)$ we denote the subsets of left and right subformulas of $G$ (a.k.a. negative/positive subformulas of $G$ [23]). Formally, $\operatorname{SL}(G)$ and $\operatorname{Sr}(G)$ are the smallest subsets of $\operatorname{Sf}(G)$ such that:

$-G \in \operatorname{SR}(G)$

- $A \odot B \in \operatorname{Sx}(G)$ implies $\{A, B\} \subseteq \operatorname{Sx}(G)$, where $\odot \in\{\wedge, \vee\}$ and $\mathrm{Sx} \in\{\mathrm{SL}, \mathrm{SR}\}$;

- $A \supset B \in \operatorname{SL}(G)$ implies $B \in \operatorname{SL}(G)$ and $A \in \operatorname{SR}(G)$;

- $A \supset B \in \operatorname{Sr}(G)$ implies $B \in \operatorname{Sr}(G)$ and $A \in \operatorname{SL}(G)$.

By $|A|$ we denote the size of $A$, namely the number of symbols in $A$. A Kripke model is a structure $\mathcal{K}=\langle P, \leq, \rho, V\rangle$, where $\langle P, \leq\rangle$ is a finite poset with minimum $\rho$ (the root of $\mathcal{K}$ ) and $V: P \rightarrow 2^{\mathcal{V}}$ is a function such that $\alpha \leq \beta$ implies $V(\alpha) \subseteq V(\beta)$. The forcing relation $\Vdash \subseteq P \times \mathcal{L}$ is defined as follows:

$-\mathcal{K}, \alpha \nVdash \perp$;

- for every $p \in \mathcal{V}, \mathcal{K}, \alpha \Vdash p$ iff $p \in V(\alpha)$;

$-\mathcal{K}, \alpha \Vdash A \wedge B$ iff $\mathcal{K}, \alpha \Vdash A$ and $\mathcal{K}, \alpha \Vdash B$;

$-\mathcal{K}, \alpha \Vdash A \vee B$ iff $\mathcal{K}, \alpha \Vdash A$ or $\mathcal{K}, \alpha \Vdash B$;

$-\mathcal{K}, \alpha \Vdash A \supset B$ iff, for every $\beta \in P$ such that $\alpha \leq \beta, \mathcal{K}, \beta \nVdash A$ or $\mathcal{K}, \beta \Vdash B$.

Monotonicity property holds for arbitrary formulas, i.e.: $\mathcal{K}, \alpha \Vdash A$ and $\alpha \leq \beta$ imply $\mathcal{K}, \beta \Vdash A$. A formula $A$ is valid in $\mathcal{K}$ iff $\mathcal{K}, \rho \Vdash A$; we say that $A$ is valid iff $A$ is valid in all the Kripke models; Intuitionistic Propositional Logic IPL coincides with the set of valid formulas 3 . If $\mathcal{K}, \rho \nVdash A$, we say that $\mathcal{K}$ is a countermodel for $A$. A final world $\gamma$ of $\mathcal{K}$ is a maximal world in $\langle P, \leq\rangle$; for every classically valid formula $A$, we have $\mathcal{K}, \gamma \Vdash A$. Let $\Gamma$ be a set of formulas, by $\mathcal{K}, \alpha \Vdash \Gamma$ we mean that $\mathcal{K}, \alpha \Vdash A$ for every $A \in \Gamma$. Using the above notation we avoid to mention the model $\mathcal{K}$ whenever it is understood (e.g., we write $\alpha \Vdash A$ instead of $\mathcal{K}, \alpha \Vdash A$ ); moreover, by "model" we mean "Kripke model".

The closure of $\Gamma$, denoted by $\mathcal{C l}(\Gamma)$, is the smallest set containing the formulas $X$ defined by the following grammar:

$$
X::=C|X \wedge X| A \vee X|X \vee A| A \supset X \quad C \in \Gamma, A \text { any formula }
$$

The following properties of closures can be easily proved:

$(\mathcal{C} l 1) \mathcal{K}, \alpha \Vdash \Gamma$ implies $\mathcal{K}, \alpha \Vdash \mathcal{C l}(\Gamma)$.

$(\mathcal{C l} 2) \quad A \in \mathcal{C} l(\Gamma)$ implies $A \in \mathcal{C} l(\Gamma \cap \operatorname{Sf}(A))$. 
$(\mathcal{C l} 3) \quad \Gamma \subseteq \mathcal{C l}(\Gamma)$ and $\mathcal{C l}(\mathcal{C l}(\Gamma))=\mathcal{C l}(\Gamma)$.

$(\mathcal{C l} 4) \Gamma_{1} \subseteq \Gamma_{2}$ implies $\mathcal{C l}\left(\Gamma_{1}\right) \subseteq \mathcal{C l}\left(\Gamma_{2}\right)$.

(Cl5) $\mathcal{C l}(\bar{\Gamma}) \cap \mathcal{V}=\Gamma \cap \mathcal{V}$.

$(\mathcal{C l 6}) \quad \Gamma_{1} \subseteq \mathcal{C l}\left(\Gamma_{2}\right)$ implies $\mathcal{C l}\left(\Gamma_{1}\right) \subseteq \mathcal{C l}\left(\Gamma_{2}\right)$ (this follows from $(\mathcal{C l})$ and $\left.(\mathcal{C l})\right)$.

\section{The calculus $\operatorname{FRJ}(G)$}

The Forward Refutation calculus FRJ $(G)$ is a calculus to infer the unprovability of a formula $G$ (the goal formula) in IPL and it is designed to support forward proof-search. The calculus acts on $\mathbf{F R J}(G)$-sequents that depend on the subformulas of $G$. Throughout the paper, we use the following notation:

$$
\bar{\Gamma}^{\mathrm{At}}=\mathrm{SL}(G) \cap \mathcal{V} \quad \bar{\Gamma}^{\supset}=\mathrm{SL}(G) \cap \mathcal{L}^{\supset} \quad \bar{\Gamma}=\bar{\Gamma}^{\mathrm{At}} \cup \bar{\Gamma}^{\supset}
$$

There are two types of $\mathbf{F R J}(G)$-sequents, we call regular (arrow $\Rightarrow$ ) and irregular (arrow $\rightarrow$ ), defined as follows:

- regular sequents have the form $\Gamma \Rightarrow C$, where $\Gamma \subseteq \bar{\Gamma}$ and $C \in \operatorname{SR}(G)$;

- irregular sequents have the form $\Sigma ; \Theta \rightarrow C$, where $\Sigma \cup \Theta \subseteq \bar{\Gamma}$ and $C \in \operatorname{SR}(G)$.

Given a sequent $\sigma$, the set Lhs $(\sigma)$ of left formulas of $\sigma$ and the right formula of $\sigma$ are defined as follows:

$$
\operatorname{Lhs}(\sigma)=\left\{\begin{array}{ll}
\Gamma & \text { if } \sigma \text { is regular } \\
\Sigma \cup \Theta & \text { if } \sigma \text { is irregular }
\end{array} \quad \operatorname{Rhs}(\sigma)=C\right.
$$

We remark that the left formulas of $\sigma$ are left subformulas of $G$ and the right formula of $\sigma$ is a right subformula of $G$. Accordingly, the number of $\mathbf{F R J}(G)$ sequents is finite, hence $\mathbf{F R J}(G)$ satisfies the Finite Rule Property [6]. Left formulas of irregular sequents $\sigma$ are partitioned into the sets $\Sigma$, the stable set of $\sigma$, and $\Theta$. In forward proof-search, formulas in $\Sigma$ are preserved as much as possible, while some of the formulas in $\Theta$ can be lost. Provability in $\mathbf{F R J}(G)$ is defined as follows:

- $\mathcal{D}$ is an $\mathbf{F R J}(G)$-derivation of $\sigma$ iff $\mathcal{D}$ is a derivation in the calculus $\mathbf{F R J}(G)$ having $\sigma$ as root sequent;

- $\mathcal{D}$ is an FRJ $(G)$-derivation of $G$ iff there exists a (possibly empty) set of formulas $\Gamma$ such that $\mathcal{D}$ is an $\mathbf{F R J}(G)$-derivation of $\Gamma \Rightarrow G$;

- $\sigma$ is provable in $\mathbf{F R J}(G)$, denoted by $\vdash_{\mathbf{F R J}(G)} \sigma$, iff there exists an $\mathbf{F R J}(G)$ derivation of $\sigma$;

- $G$ is provable in $\mathbf{F R J}(G)$, denoted by $\vdash_{\mathbf{F R J}(G)} G$, iff there exists an $\mathbf{F R J}(G)$ derivation of $G$.

Soundness of $\mathbf{F R J}(G)$ is stated as follows:

Theorem 1 (Soundness of FRJ $(G)$ ). $\vdash_{\mathbf{F R J}(G)} G$ implies $G \notin \mathrm{IPL}$.

Soundness of $\mathbf{F R J}(G)$ relies on the fact that rules of $\mathbf{F R J}(G)$ satisfy the following soundness properties: 
(S1) if $\Gamma \Rightarrow C$ is provable in $\mathbf{F R J}(G)$, then there exists a world $\alpha$ of a model $\mathcal{K}$ such that $\alpha \Vdash \Gamma$ and $\alpha \nVdash C$;

(S2) if $\sigma=\Sigma ; \Theta \rightarrow C$ is provable in $\mathbf{F R J}(G)$ and $\sigma$ can be used to (directly or indirectly) prove a regular sequent in $\mathbf{F R J}(G)$, then there exist a world $\alpha$ of a model $\mathcal{K}$ and a set $\Gamma$ such that $\Sigma \subseteq \Gamma \subseteq \Sigma \cup \Theta$ and $\alpha \Vdash \Gamma$ and $\alpha \nVdash C$.

Properties (S1) and (S2) follow by Lemma 2 of Sec. 3.2 Note that soundness hides subsumption, which is typical of forward reasoning. Indeed, let $G$ be provable in $\mathbf{F R J}(G)$. Then, there exists an $\mathbf{F R J}(G)$-derivation of a sequent $\Gamma \Rightarrow G$. Property (S1) implies that the formula $(\wedge \Gamma) \supset G$ is not valid (equivalently, $G$ is not provable from assumptions $\Gamma$ ).

The calculus $\mathbf{F R J}(G)$ consists of two axiom rules, some right introduction rules for the connectives $\wedge, \vee, \supset$ and the rules $\bowtie^{\text {At }}$ and $\bowtie^{\vee}$ to join sequents; there are no left rules. Rules of $\mathbf{F R J}(G)$ are collected in Fig. 1. below we provide an in-depth presentation.

Axiom rules We have two axiom rules:

$$
\overline{\bar{\Gamma}^{\mathrm{At}} \backslash\{F\} \Rightarrow F} \mathrm{Ax}_{\Rightarrow} \quad \overline{\cdot ; \bar{\Gamma}^{\mathrm{At}} \backslash\{F\}, \bar{\Gamma}^{\supset} \rightarrow F} \mathrm{Ax}_{\rightarrow} \quad F \in \mathcal{V}^{\perp}
$$

Rule $\mathrm{Ax}_{\Rightarrow}$ introduces a regular axiom, rule $\mathrm{Ax}_{\rightarrow}$ an irregular one. Both axiom sequents have in the right a formula $F \in \mathcal{V}^{\perp}$; in irregular axioms the set $\Sigma$ is empty (denoted by $\cdot$ ). We recall that forward proof-search starts from axiom sequents.

Rules for $\wedge$ We have two rules to introduce $\wedge$ in the right, one concerning regular sequents and one the irregular ones. In both rules the type of the sequents (regular or irregular) does not change:

$$
\frac{\Gamma \Rightarrow A_{k}}{\Gamma \Rightarrow A_{1} \wedge A_{2}} \wedge \quad \frac{\Sigma ; \Theta \rightarrow A_{k}}{\Sigma ; \Theta \rightarrow A_{1} \wedge A_{2}} \wedge k \in\{1,2\}
$$

Rules for $\supset$ In standard refutation calculi, the rule for right implication is

$$
\frac{\Gamma \Rightarrow B}{\Gamma \Rightarrow A \supset B} R \supset \quad A \in \Gamma
$$

The side condition $A \in \Gamma$ is needed to guarantee that, assuming that $B$ is not provable from $\Gamma$, then $A \supset B$ is not provable from $\Gamma$ as well. With such a rule alone, the calculus $\mathbf{F R J}(G)$ would be incomplete. For instance, using the rules presented so far, we are not able to derive the goal $G=p_{1} \wedge p_{2} \supset q$. Indeed, let us start from the axiom $p_{1}, p_{2} \Rightarrow q$. We can build the derivations

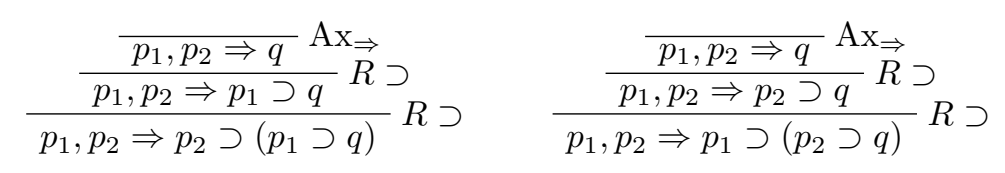


but there is no means to obtain $G$ in the right, since the formula $p_{1} \wedge p_{2}$ is not allowed in the left of sequents. To compensate for this, we have to relax the side condition. This leads to the rule $\supset \in$ of $\mathbf{F R J}(G)$

$$
\frac{\Gamma \Rightarrow B}{\Gamma \Rightarrow A \supset B} \supset \in \quad A \in \mathcal{C l}(\Gamma)
$$

where we introduce the more general side condition $A \in \mathcal{C l}(\Gamma)$ (whence the $\epsilon$ in the rule name). Using this rule, we can prove the goal $G=p_{1} \wedge p_{2} \supset q$ as follows:

$$
\frac{\frac{p_{1}, p_{2} \Rightarrow q}{p_{1}, p_{2} \Rightarrow p_{1} \wedge p_{2} \supset q}}{p_{\epsilon}} \supset_{\in} \quad p_{1} \wedge p_{2} \in \mathcal{C l}\left(\left\{p_{1}, p_{2}\right\}\right)
$$

We can also apply rule $\supset_{\in}$ to an irregular sequent $\sigma_{1}=\Sigma ; \Theta^{\prime} \rightarrow B$ to get an irregular sequent $\sigma=\Sigma^{\prime} ; \Theta \rightarrow A \supset B$. In this case the antecedent $A$ must belong to $\mathcal{C l}\left(\Sigma^{\prime}\right)$ and we are allowed to transfer formulas from $\Theta^{\prime}$ to $\Sigma^{\prime}$ so to satisfy such a condition. We can formalize rule $\supset_{\in}$ for irregular sequents as follows:

$$
\frac{\sigma_{1}=\Sigma ; \overbrace{\Theta, \Lambda}^{\Theta^{\prime}} \rightarrow B}{\sigma=\underbrace{\Sigma, \Lambda}_{\Sigma^{\prime}} ; \Theta \rightarrow A \supset B} \supset \in \quad \begin{array}{ll}
\Theta \cap \Lambda=\mathcal{C l}(\Sigma \cup \Lambda) \\
A, \Theta
\end{array}
$$

The set $\Theta^{\prime}$ has been partitioned as $\Theta \cup \Lambda$, where the (possibly empty) set $\Lambda$ is shifted to the left of semicolon; note that $\operatorname{Lhs}(\sigma)=\operatorname{Lhs}\left(\sigma_{1}\right)$. We remark that, to get the formula $A \supset B$ in the conclusion, in general many choices of $\Lambda$ are possible, as illustrated in the next example.

Example 1. Let $\sigma_{1}=\cdot ; p, q \rightarrow B$ be an $\mathbf{F R J}(G)$-sequent such that the formula $p \vee q \supset B$ belongs to $\operatorname{SR}(G)$. To apply rule $\supset \in$ to $\sigma_{1}$ so to get a sequent with $p \vee q \supset B$ in the right, we have to select a subset $\Lambda$ of $\{p, q\}$ satisfying the side condition $p \vee q \in \mathcal{C l}(\Lambda)$. The following three choices are possible:

$$
\begin{array}{lll}
\Lambda_{1}=\{p\} & \Lambda_{2}=\{q\} & \Lambda_{3}=\{p, q\} \\
\frac{\cdot ; p, q \rightarrow B}{p ; q \rightarrow p \vee q \supset B} \supset_{\in} & \frac{\cdot ; p, q \rightarrow B}{q ; p \rightarrow p \vee q \supset B} \supset_{\in} & \frac{\cdot ; p, q \rightarrow B}{p, q ; \cdot \rightarrow p \vee q \supset B} \supset_{\epsilon}
\end{array}
$$

We point out that $\Lambda_{1}$ and $\Lambda_{2}$ are minimal sets satisfying the side condition, while $\Lambda_{3}$ is not. Indeed, the empty set is the only proper subset of $\Lambda_{1}$ and $\Lambda_{2}$ and $p \vee q \notin \mathcal{C l}(\emptyset)$. On the other hand, $\Lambda_{3}$ is not minimal, since both $\Lambda_{1}$ and $\Lambda_{2}$ are proper subsets of $\Lambda_{3}$.

We need a further rule to introduce $A \supset B$ in the right, having premise $\sigma_{1}=\Gamma \Rightarrow B$ and conclusion $\sigma=\cdot ; \Theta \rightarrow A \supset B$; this is the only rule of $\mathbf{F R J}(G)$ turning a regular sequent into an irregular one. We require that $A \in \mathcal{C l}(\Gamma)$ and $\Theta$ is any subset of $\mathcal{C l}(\Gamma) \cap \bar{\Gamma}$ such that $A \notin \mathcal{C l}(\Theta)$. By the latter condition, we call the rule $\supset_{\notin}$ :

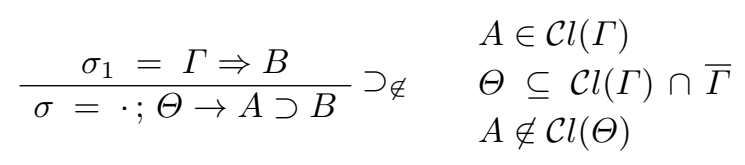


The condition $A \notin \mathcal{C l}(\Theta)$ is essentially needed to introduce a decreasing weight function on sequents (see Sec. 3.2). Also in this case, many choices of $\Theta$ are possible in general, as shown in the next example.

Example 2. Let $\sigma_{1}=p, q \Rightarrow B$ be an $\mathbf{F R J}(G)$-sequent and let assume that

$\bar{\Gamma}=\operatorname{SL}(G) \cap\left(\mathcal{V} \cup \mathcal{L}^{\supset}\right)=\{p, q, r, r \supset p, p \supset r\} \quad C=p \wedge q \supset B \in \operatorname{SR}(G)$

We show all the possible applications of $\supset_{\notin}$ to $\sigma_{1}$ yielding an irregular sequent of the form $\cdot ; \Theta \rightarrow C$. By the side conditions, $\Theta$ must be a subset of $\mathcal{C l}(\{p, q\}) \cap \bar{\Gamma}=$ $\{p, q, r \supset p\}$, which gives rise to eight possible choices:
$\Theta_{1}=\emptyset$
$\Theta_{2}=\{p\}$
$\Theta_{3}=\{q\}$
$\Theta_{4}=\{r \supset p\}$
$\Theta_{5}=\{p, r \supset p\}$
$\Theta_{6}=\{q, r \supset p\}$
$\Theta_{7}=\{p, q\}$
$\Theta_{8}=\{p, q, r \supset p\}$

By the side conditions, we need $p \wedge q \notin \mathcal{C l}(\Theta)$, hence $\Theta_{7}$ and $\Theta_{8}$ must be ruled out. This leads to six possible applications of rule $\supset_{\notin}$ :

$$
\begin{aligned}
& \Theta_{1}=\emptyset \frac{p, q \Rightarrow B}{\cdot ; \Theta_{1} \rightarrow C} \supset_{\notin} \quad \Theta_{2}=\{p\} \quad \frac{p, q \Rightarrow B}{\cdot ; \Theta_{2} \rightarrow C} \supset_{\notin} \\
& \Theta_{3}=\{q\} \quad \frac{p, q \Rightarrow B}{\cdot ; \Theta_{3} \rightarrow C} \supset_{\notin} \quad \Theta_{4}=\{r \supset p\} \quad \frac{p, q \Rightarrow B}{\cdot ; \Theta_{4} \rightarrow C} \supset_{\notin} \\
& \Theta_{5}=\{p, r \supset p\} \quad \frac{p, q \Rightarrow B}{\cdot ; \Theta_{5} \rightarrow C} \supset_{\notin} \quad \Theta_{6}=\{q, r \supset p\} \quad \frac{p, q \Rightarrow B}{\cdot ; \Theta_{6} \rightarrow C} \supset_{\notin}
\end{aligned}
$$

We point out that $\Theta_{5}$ is a maximal set satisfying the side condition. Indeed, the only proper superset $\Theta^{\prime}$ of $\Theta_{5}$ such that $\Theta^{\prime} \subseteq \mathcal{C} l(\{p, q\}) \cap \bar{\Gamma}$ is $\Theta_{8}$ and $p \wedge q \in$ $\mathcal{C l}\left(\Theta_{8}\right)$. Similarly, $\Theta_{6}$ is maximal as well. On the other hand, sets $\Theta_{1}, \ldots, \Theta_{4}$ are not maximal, since each of them is a proper subset of $\Theta_{5}$ or $\Theta_{6}$.

Rule $\vee$ The rule $\vee$ has premises $\sigma_{1}=\Sigma_{1} ; \Theta_{1} \rightarrow C_{1}$ and $\sigma_{2}=\Sigma_{2} ; \Theta_{2} \rightarrow C_{2}$ and conclusion $\sigma=\Sigma ; \Theta \rightarrow C_{1} \vee C_{2}$ introducing a disjunction in the right. As discussed in the Introduction, some care is required in defining the left formulas of $\sigma$. The leading idea is that the $\Sigma$-sets of premises must be preserved in the conclusion, while the $\Theta$-sets are intersected. We have to guarantee that $\operatorname{Lhs}(\sigma) \subseteq$ $\operatorname{Lhs}\left(\sigma_{1}\right) \cap \operatorname{Lhs}\left(\sigma_{2}\right)$, hence some side conditions on the $\Sigma$-sets are needed. We define:

$$
\frac{\sigma_{1}=\Sigma_{1} ; \Theta_{1} \rightarrow C_{1} \quad \sigma_{2}=\Sigma_{2} ; \Theta_{2} \rightarrow C_{2}}{\sigma=\underbrace{\Sigma_{1}, \Sigma_{2}}_{\Sigma} ; \underbrace{\Theta_{1} \cap \Theta_{2}}_{\Theta} \rightarrow C_{1} \vee C_{2}} \vee \begin{aligned}
& \Sigma_{1} \subseteq \Sigma_{2} \cup \Theta_{2} \\
& \Sigma_{2} \subseteq \Sigma_{1} \cup \Theta_{1}
\end{aligned}
$$

Join rules The join rules $\bowtie^{\mathrm{At}}$ and $\bowtie^{\vee}$ apply to $n \geq 1$ irregular sequents $\sigma_{1}=$ $\Sigma_{1} ; \Theta_{1} \rightarrow A_{1}, \ldots, \sigma_{n}=\Sigma_{n} ; \Theta_{n} \rightarrow A_{n}$ and yield a regular sequent $\sigma=\Gamma \Rightarrow C$; this is the only way to obtain a regular sequent from irregular ones. These two 
rules have a similar structure and only differ in the form of $C$ : in rule $\bowtie^{\mathrm{At}}$, $C \in \mathcal{V}^{\perp}$ while in $\star^{\vee}, C$ is an $\vee$-formula. For every $1 \leq j \leq n$, we write the premise $\sigma_{j}$ as follows:

$$
\sigma_{j}=\underbrace{\Sigma_{j}^{\mathrm{At}}, \Sigma_{j}^{\supset}}_{\Sigma_{j}} ; \underbrace{\Theta_{j}^{\mathrm{At}}, \Theta_{j}^{\supset}}_{\Theta_{j}} \rightarrow A_{j} \quad \Sigma_{j}^{\mathrm{At}} \cup \Theta_{j}^{\mathrm{At}} \subseteq \mathcal{V} \text { and } \Sigma_{j}^{\supset} \cup \Theta_{j}^{\supset} \subseteq \mathcal{L}^{\supset}
$$

Similarly to rule $\vee$, formulas in $\Sigma_{j}$ must be preserved in the conclusion and we need $\operatorname{Lhs}(\sigma) \subseteq \operatorname{Lhs}\left(\sigma_{1}\right) \cap \cdots \cap \operatorname{Lhs}\left(\sigma_{n}\right)$. Thus, we require the side condition of rule $\vee$ for every pair of distinct premises, namely:

(J1) $\Sigma_{i} \subseteq \Sigma_{j} \cup \Theta_{j}$ for every $i \neq j$.

From a semantic perspective, the role of join rules is to downward expand a countermodel under construction; the conclusion $\sigma=\Gamma \Rightarrow C$ directly corresponds to a new world $\alpha$ of the countermodel such that all the formulas in $\Gamma$ are forced in $\alpha$ and $C$ is not forced in $\alpha$ (see the soundness property (S1)]. To perform this, we need a further side condition (J2) on the sets $\Sigma_{j}$. Let

$$
\Sigma^{\mathrm{At}}=\bigcup_{1 \leq j \leq n} \Sigma_{j}^{\mathrm{At}} \quad \Sigma^{\supset}=\bigcup_{1 \leq j \leq n} \Sigma_{j}^{\supset}
$$

Since both $\Sigma^{\text {At }}$ and $\Sigma^{\supset}$ must be kept in the conclusion $\sigma=\Gamma \Rightarrow C$ (namely, $\Sigma^{\mathrm{At}} \cup \Sigma^{\supset} \subseteq \Gamma$ ), we need that all the formulas in $\Sigma^{\mathrm{At}} \cup \Sigma^{\supset}$ are forced in the new world $\alpha$. A formula $Y \supset Z$ is supported if there exists a premise $\sigma_{k}(1 \leq k \leq n)$ such that $Y=A_{k}\left(A_{k}\right.$ is the right formula of $\left.\sigma_{k}\right)$. Intuitively, if a formula $Y \supset Z \in \Sigma^{\supset}$ is supported, then $Y$ is not forced in $\alpha$, and this allows us to conclude that $Y \supset Z$ is forced in $\alpha$. We require that all the $\supset$-formulas in the sets $\Sigma_{j}$ are supported, and this is formalized by the following side condition:

(J2) $Y \supset Z \in \Sigma^{\supset}$ implies $Y \in\left\{A_{1}, \ldots, A_{n}\right\}$.

Example 3. Let $\sigma_{1}=\Sigma_{1} ; \Theta_{1} \rightarrow B$, where $\Sigma_{1}=\left\{B \supset X_{1}, B \supset X_{2}, C \supset X_{3}\right\}$ and $C \neq B$. We cannot apply a join rule having $\sigma_{1}$ as only premise since the formula $C \supset X_{3}$ would not be supported (while both $B \supset X_{1}$ and $B \supset X_{2}$ are, being $B$ the right formula of $\sigma_{1}$ ). Thus, to apply a join rule, we need a further premise $\sigma_{2}=\Sigma_{2} ; \Theta_{2} \rightarrow C$ such that $\Sigma_{1} \subseteq \Sigma_{2} \cup \Theta_{2}$ and $\Sigma_{2} \subseteq \Sigma_{1} \cup \Theta_{1}$ (see((J1)). In turn, $\Sigma_{2}$ might contain some non-supported formulas; e.g., a formula $D \supset X_{4}$ such that $D \neq B$ and $D \neq C$; in this case, we need a further premise $\sigma_{3}$ s.t. $\operatorname{Rhs}\left(\sigma_{3}\right)=D$ to support it.

Side conditions do not concern the sets $\Theta_{j}$ and in the conclusion we keep some of the formulas in the intersection of all the $\Theta_{j}$ 's. More precisely, given a set of $\supset$-formulas $\Gamma^{\supset}$ and a set of formulas $\Upsilon$, let

$$
\Gamma^{\supset} / \Upsilon=\left\{Y \supset Z \in \Gamma^{\supset} \mid Y \in \Upsilon\right\}
$$


We call $\Gamma^{\supset} / \Upsilon$ the restriction of $\Gamma^{\supset}$ to $\Upsilon$. Then, the formulas $\Theta^{\text {At }}$ and $\Theta^{\supset}$ to be kept in the conclusion are defined as follows:

$$
\Theta^{\mathrm{At}}=\bigcap_{1 \leq j \leq n} \Theta_{j}^{\mathrm{At}} \quad \Theta^{\supset}=\left(\bigcap_{1 \leq j \leq n} \Theta_{j}^{\supset}\right) /\left\{A_{1}, \ldots, A_{n}\right\}
$$

We can now define the conclusion $\sigma$ of a join rule having premises $\sigma_{1}, \ldots, \sigma_{n}$ matching the side conditions $(\mathrm{J} 1)$ and $(\mathrm{J} 2)$. In rule $\bowtie^{\mathrm{At}}$, we can choose as right formula of $\sigma$ any formula $F \in \mathcal{V}^{\perp} \cap \operatorname{Rhs}(G)$ such that $F \notin \Sigma^{\text {At }}$. We set:

$$
\sigma=\Sigma^{\mathrm{At}}, \Theta^{\mathrm{At}} \backslash\{F\}, \Sigma^{\supset}, \Theta^{\supset} \Rightarrow F
$$

In rule $\bowtie^{\vee}$, we can choose as right formula of $\sigma$ any $\vee$-formula $C_{1} \vee C_{2} \in \operatorname{Rhs}(G)$ such that both $C_{1}$ and $C_{2}$ are among $A_{1}, \ldots, A_{n}$. We set:

$$
\sigma=\Sigma^{\mathrm{At}}, \Theta^{\mathrm{At}}, \Sigma^{\supset}, \Theta^{\supset} \Rightarrow C_{1} \vee C_{2} \quad\left\{C_{1}, C_{2}\right\} \subseteq\left\{A_{1}, \ldots, A_{n}\right\}
$$

We note that rule $\bowtie^{\vee}$ is similar to the rule $r_{n}$ presented in [21].

We point out the following special case of application of $\bowtie^{\text {At }}$ having only one premise ( $\Sigma^{\supset}$ is possibly empty):

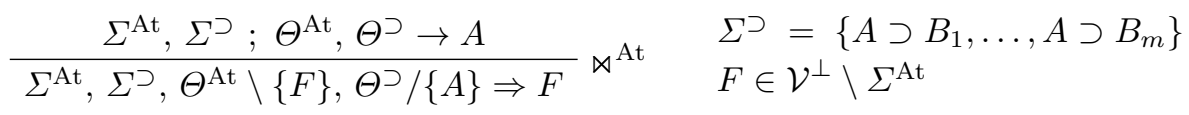

Now, we begin to study the properties of $\mathbf{F R J}(G)$-derivations. Let us introduce the following relations between sequents of $\mathbf{F R J}(G)$ :

- $\sigma_{1} \stackrel{\mathcal{R}}{\mapsto} \sigma_{0}$ iff $\mathcal{R}$ is a rule of $\mathbf{F R J}(G)$ such that $\sigma_{2}$ is the conclusion of $\mathcal{R}$ and $\sigma_{1}$ is one of its premises;

- $\sigma_{1} \mapsto_{0} \sigma_{2}$ iff there exists a rule $\mathcal{R}$ of $\mathbf{F R J}(G)$ such that $\sigma_{1} \stackrel{\mathcal{R}}{\mapsto_{0}} \sigma_{2}$;

$-\mapsto$ is the transitive closure of $\mapsto_{0}$;

$-\mapsto_{*}$ is the reflexive closure of $\mapsto$.

By inspecting the rules of $\mathbf{F R J}(G)$ and exploiting properties $(\mathcal{C} l 3)$ and $(\mathcal{C} l 6)$ of closures, the following facts can be easily proved:

\section{Lemma 1.}

(i) $\sigma_{1} \stackrel{\mathcal{R}}{\mapsto}_{0} \sigma_{2}$ and $\mathcal{R} \neq \supset_{\sharp}$ imply $\operatorname{Lhs}\left(\sigma_{2}\right) \subseteq \operatorname{Lhs}\left(\sigma_{1}\right)$.

(ii) $\sigma_{1} \mapsto_{0} \sigma_{2}$ implies $\operatorname{Lhs}\left(\sigma_{2}\right) \subseteq \mathcal{C l}\left(\operatorname{Lhs}\left(\sigma_{1}\right)\right)$.

(iii) $\sigma_{1} \mapsto_{*} \sigma_{2}$ implies $\operatorname{Lhs}\left(\sigma_{2}\right) \subseteq \mathcal{C l}\left(\operatorname{Lhs}\left(\sigma_{1}\right)\right)$.

In the next example we show that the unsound derivation discussed in the Introduction cannot be simulated in $\mathbf{F R J}(G)$. 


$$
\begin{aligned}
& \bar{\Gamma}^{\mathrm{At}}=\mathrm{SL}(G) \cap \mathcal{V}, \bar{\Gamma}^{\supset}=\mathrm{SL}(G) \cap \mathcal{L}^{\supset}, \bar{\Gamma}=\bar{\Gamma}^{\mathrm{At}} \cup \bar{\Gamma}^{\supset} . \\
& \text { In the conclusion } \sigma \text { of each rule, } \operatorname{Rhs}(\sigma) \in \operatorname{SR}(G) \\
& \overline{\bar{\Gamma}^{\mathrm{At}} \backslash\{F\} \Rightarrow F} \mathrm{Ax} \Rightarrow \quad \overline{\cdot ; \bar{\Gamma}^{\mathrm{At}} \backslash\{F\}, \bar{\Gamma}^{\supset} \rightarrow F} \mathrm{Ax} \rightarrow \quad F \in \mathcal{V}^{\perp} \\
& \frac{\Gamma \Rightarrow A_{k}}{\Gamma \Rightarrow A_{1} \wedge A_{2}} \wedge \quad \frac{\Sigma ; \Theta \rightarrow A_{k}}{\Sigma ; \Theta \rightarrow A_{1} \wedge A_{2}} \wedge k \in\{1,2\} \\
& \frac{\Sigma_{1} ; \Theta_{1} \rightarrow C_{1} \quad \Sigma_{2} ; \Theta_{2} \rightarrow C_{2}}{\Sigma_{1}, \Sigma_{2} ; \Theta_{1} \cap \Theta_{2} \rightarrow C_{1} \vee C_{2}} \vee \quad \begin{array}{l}
\Sigma_{1} \subseteq \Sigma_{2} \cup \Theta_{2} \\
\Sigma_{2} \subseteq \Sigma_{1} \cup \Theta_{1}
\end{array} \\
& \frac{\Gamma \Rightarrow B}{\Gamma \Rightarrow A \supset B} \supset \in \quad A \in \mathcal{C} l(\Gamma) \quad \frac{\Sigma ; \Theta, \Lambda \rightarrow B}{\Sigma, \Lambda ; \Theta \rightarrow A \supset B} \supset \in \begin{array}{l}
\Theta \cap \Lambda=\emptyset \\
A \in \mathcal{C} l(\Sigma \cup \Lambda)
\end{array} \\
& \begin{aligned}
\frac{\Gamma \Rightarrow B}{\cdot ; \Theta \rightarrow A \supset B} \supset \notin & \Theta \subseteq \mathcal{C l}(\Gamma) \cap \bar{\Gamma} \\
& A \in \mathcal{C l}(\Gamma) \backslash \mathcal{C} l(\Theta)
\end{aligned} \\
& \text { Let, for } 1 \leq j \leq n, \sigma_{j}=\underbrace{\Sigma_{j}^{\mathrm{At}}, \Sigma_{j}^{\supset}}_{\Sigma_{j}} ; \underbrace{\Theta_{j}^{\mathrm{At}}, \Theta_{j}^{\supset}}_{\Theta_{j}} \rightarrow A_{j} \text { and } \Upsilon=\left\{A_{1}, \ldots, A_{n}\right\} \\
& \Sigma^{\mathrm{At}}=\bigcup_{1 \leq j \leq n} \Sigma_{j}^{\mathrm{At}} \quad \Theta^{\mathrm{At}}=\bigcap_{1 \leq j \leq n} \Theta_{j}^{\mathrm{At}} \quad \Sigma^{\supset}=\bigcup_{1 \leq j \leq n} \Sigma_{j}^{\supset} \quad \Theta^{\supset}=\left(\bigcap_{1 \leq j \leq n} \Theta_{j}^{\supset}\right) / \Upsilon
\end{aligned}
$$

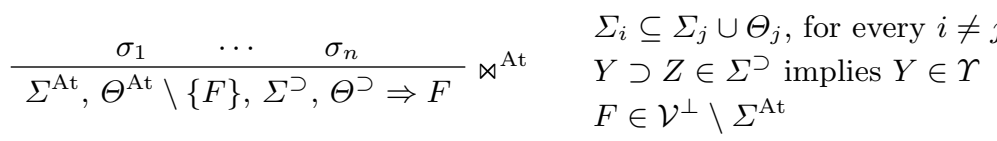

$$
\begin{aligned}
& \begin{array}{cl}
\frac{\sigma_{1} \quad \cdots \quad \sigma_{n}}{\Sigma^{\mathrm{At}}, \Theta^{\mathrm{At}}, \Sigma^{\supset}, \Theta^{\supset} \Rightarrow C_{1} \vee C_{2}} \bowtie^{\vee} & \Sigma_{i} \subseteq \Sigma_{j} \cup \Theta_{j}, \text { for every } i \neq j \\
& Y \supset Z \in \Sigma^{\supset} \text { implies } Y \in \Upsilon \\
& \left\{C_{1}, C_{2}\right\} \subseteq \Upsilon
\end{array}
\end{aligned}
$$

Fig. 1. The calculus FRJ $(G)$.

Example 4. In the Introduction, by applying the unsound rule $R \vee$, we got a wrong derivation of the sequent $\sigma=p, H \Rightarrow q_{1} \vee q_{2}$, where $H=p \supset q_{1} \vee q_{2}$. By the soundness property (S1), if $\sigma$ could be proved in $\mathbf{F R J}(G)$, there should be a world $\alpha$ of a model such that both $p$ and $H$ are forced in $\alpha$ and $q_{1} \vee q_{2}$ is not forced in $\alpha$, a contradiction. We show that it is not possible to build an $\operatorname{FRJ}(G)$-derivation $\mathcal{D}$ of $\sigma$. Indeed, the root rule of $\mathcal{D}$ should be $\star^{\vee}$, the only rule of $\mathbf{F R J}(G)$ having as conclusion a regular sequent with an $\vee$-formula in the right. Since the formula $H$ must be supported (see (J2)], rule $\bowtie^{\vee}$ should have a premise $\sigma^{\prime}=\Sigma^{\prime} ; \Theta^{\prime} \rightarrow p$. Since the only irregular sequents having a formula in 
$\mathcal{V}^{\perp}$ in the right are the irregular axioms, $\mathcal{D}$ should have the following shape:

$$
\mathcal{D}=\frac{\cdots \quad \overline{\sigma^{\prime}=\cdot ; \Theta^{\prime} \rightarrow p} \mathrm{Ax}_{\rightarrow} \quad \cdots}{\sigma=p, H \Rightarrow q_{1} \vee q_{2}} \bowtie^{\vee} \quad p \notin \Theta^{\prime}
$$

Hence, $\sigma^{\prime} \stackrel{\aleph^{\vee}}{\mapsto_{0}} \sigma$ which, by Lemma 1 (i) implies $\{p, H\} \subseteq \Theta^{\prime}$. Thus, both $p \in \Theta^{\prime}$ and $p \notin \Theta^{\prime}$, a contradiction. We conclude that $\sigma$ is not provable.

However, it is possible to have applications of rule $\bowtie^{\vee}$ having conclusion $p \Rightarrow q_{1} \vee q_{2}$ or $H \Rightarrow q_{1} \vee q_{2}$. For instance, let

$$
G=(p \wedge H) \supset\left(q_{1} \vee q_{2}\right) \quad H=p \supset q_{1} \vee q_{2}
$$

We have:

$$
\mathrm{SL}(G)=\left\{p \wedge H, H, q_{1} \vee q_{2}, p, q_{1}, q_{2}\right\} \quad \operatorname{SR}(G)=\left\{G, q_{1} \vee q_{2}, p, q_{1}, q_{2}\right\}
$$

We can build the following $\mathbf{F R J}(G)$-derivation:

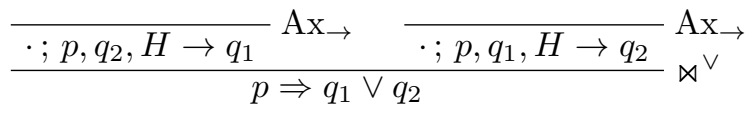

We point out that the side conditions $(\mathrm{J} 1)$ and $(\mathrm{J} 2)$ trivially hold, since the $\Sigma$-sets of the premises are empty. In the conclusion, $H$ is left out since it is not supported (no premise has $p$ in the right). Similarly, we can build the following $\operatorname{FRJ}(G)$-derivation:

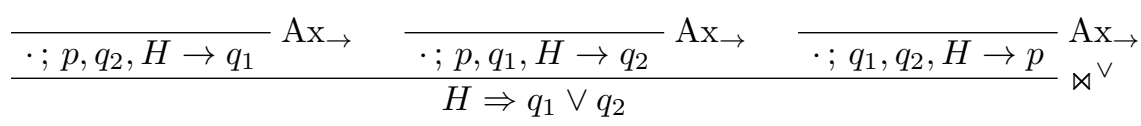

In the conclusion, $p$ is omitted since it does not occur as left formula in the right-most premise. We can also build the following $\mathbf{F R J}(G)$-derivation of an irregular sequent $\sigma_{1}$ having the goal formula $G$ in the right:

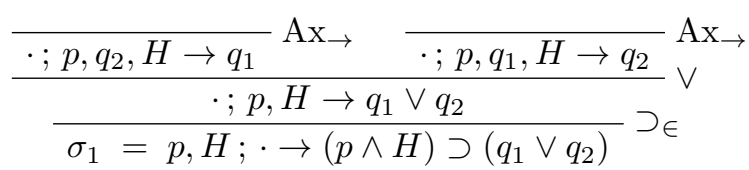

Apparently, this contradicts the soundness of the calculus, since the formula $G$ is valid. Actually, we conclude that the hypothesis of the soundness property (S2) does not hold, namely, $\sigma_{1}$ cannot be used to derive in $\mathbf{F R J}(G)$ a regular sequent.

Hereafter, to reduce the proof-search space, we assume that $\mathbf{F R J}(G)$-derivations comply with the following restrictions (PS1) (PS4).

(PS1) In rule $\supset_{\epsilon}$, we require that $\Lambda$ is a minimal set satisfying the side condition, namely: $\Lambda^{\prime} \subsetneq \Lambda$ implies $A \notin \mathcal{C l}\left(\Sigma \cup \Lambda^{\prime}\right)$. 


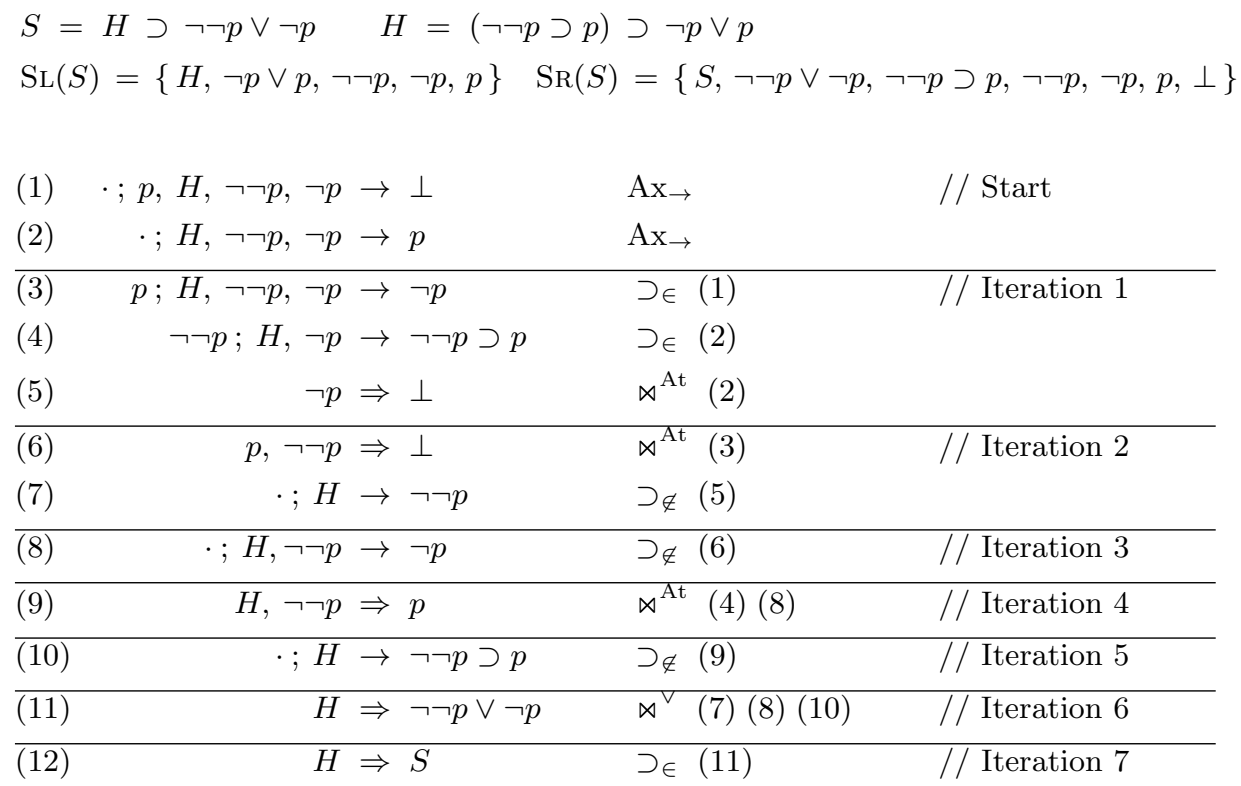

Fig. 2. The $\mathbf{F R J}(S)$-derivation $\mathcal{D}_{S}$ of $S$ and the model $\operatorname{Mod}\left(\mathcal{D}_{S}\right)$.

(PS2) In rule $\supset_{\notin}$, we require that $\Theta$ is a maximal set satisfying the side condition, namely: $\Theta \subsetneq \Theta^{\prime} \subseteq \mathcal{C l}(\Gamma) \cap \bar{\Gamma}$ implies $A \in \mathcal{C} l\left(\Theta^{\prime}\right)$.

(PS3) In $\star^{\text {At }}$, for every $Y \in \Upsilon$ there is $Y \supset Z \in \operatorname{SL}(G)$.

(PS4) In $\star^{\vee}$, for every $Y \in \Upsilon$, either there is $Y \supset Z \in \operatorname{SL}(G)$ or there is $Y \vee Z \in \operatorname{SR}(G)$ or there is $Z \vee Y \in \operatorname{SR}(G)$.

In Ex. 1. the applications of $\supset \in$ complying with (PS1) are the ones concerning $\Lambda_{1}$ and $\Lambda_{2}$; In Ex. 2. the applications of $\supset_{\notin}$ satisfying[(PS2) are the ones related to $\Theta_{5}$ and $\Theta_{6}$.

We now provide some significant examples of derivations.

Example 5. Let us consider the following instances $S$ and $T$ of Scott and Anti$S$ cott principles, which are equivalent to Nishimura formulas $N_{10}$ and $N_{9}$ respectively [3] (the schema generating $N_{i}$ is given in Sec. 7):

$$
S=((\neg \neg p \supset p) \supset \neg p \vee p) \supset \neg \neg p \vee \neg p \quad T=S \supset(\neg \neg p \supset p) \vee \neg \neg p
$$

Both formulas are valid in Classical Logic but not in IPL. Figs. 2 and 3 show an FRJ $(S)$-derivation $\mathcal{D}_{S}$ of $S$ and an $\mathbf{F R J}(T)$-derivation $\mathcal{D}_{T}$ of $T$ respectively, in linear representation. We populate the database of proved sequents according with the naive recipe of [6]: we start by inserting the axioms; then we enter a loop where, at each iteration, we apply the rules to the sequents collected in previous 
steps. For the sake of conciseness, we only show the sequents needed to get the goal. We denote with $\sigma_{(k)}$ the sequent derived at line $(k)$. The tree-like structure of derivations $\mathcal{D}_{S}$ and $\mathcal{D}_{T}$ are displayed in Figs. 5 and 6 respectively. We point out that $\mathcal{D}_{T}$ contains an application of $\bowtie^{\vee}$ having four premises, namely:

$$
\begin{aligned}
& \frac{\sigma[2 \quad \sigma \sqrt{7} \quad \sigma[8}{\sigma \sqrt{13}} \bowtie^{\vee} \\
& \sigma_{2}=\cdot ; S, \neg \neg p \supset p, \neg \neg p, \neg p \rightarrow p \quad \sigma(7)=\cdot ; S, \neg \neg p \supset p \rightarrow \neg \neg p \\
& \sigma \sqrt{8}=\cdot ; S, \neg \neg p \supset p, \neg \neg p \rightarrow \neg p \quad \sigma \sqrt{11}=\neg \neg p \supset p ; S, \neg \neg p \rightarrow H \\
& \sigma \sqrt{13}=\neg \neg p \supset p, S \Rightarrow \neg p \vee p \quad S=H \supset \neg \neg p \vee \neg p
\end{aligned}
$$

The sequent $\sigma_{13}$ is essential to build the countermodel since it corresponds to a world where both $\neg \neg p \supset p$ and $S$ are forced, while $\neg p \vee p$ is not; to get $\Gamma=\{\neg \neg p \supset p, S\}$ in the left of $\sigma_{(13 p}$, we have to use premises $\sigma^{\prime}$ such that $\Gamma \subseteq \operatorname{Lhs}\left(\sigma^{\prime}\right)$ (see Lemma 1 $1(\mathrm{i})$. Sequents $\sigma_{8,}$ and $\sigma_{2}$ are needed to obtain $\neg p \vee p$ in the right of $\sigma_{133}$, while sequents $\sigma_{77}$ and $\sigma_{111}$ are needed to support $\neg \neg p \supset p$ and $S$ respectively. One can easily check that the side conditions (J1) and (J2) hold, thus the displayed application of rule $\bowtie^{\vee}$ is sound. Finally, we point out that it is not possible to obtain $\sigma_{13}$ using less than four premises. $\diamond$

Example 6. Another significant example is the $\mathbf{F R J}(K)$-derivation $\mathcal{D}_{K}$ of the instance $K=(\neg a \supset b \vee c) \supset(\neg a \supset b) \vee(\neg a \supset c)$ of Kreisel-Putnam principle [3] shown in Fig. 4 . Differently from the formulas $S$ and $T$ in the previous example, the formula $K$ contains three propositional variables, which give rise to eight axioms. In the figure, we only consider the sequents needed to prove the goal. $\diamond$

\subsection{Countermodels and soundness of $\operatorname{FRJ}(G)$}

We show that we can extract from an $\mathbf{F R J}(G)$-derivation $\mathcal{D}$ of $G$ a countermodel for $G$. We call $p$-sequent (prime sequent) of $\mathcal{D}$ any regular sequent occurring in $\mathcal{D}$ which is either an axiom or the conclusion of a join rule. Given an $\mathbf{F R J}(G)$ derivation $\mathcal{D}$ of $G$ let $\operatorname{Mod}(\mathcal{D})=\langle\mathrm{P}(\mathcal{D}), \leq, \rho, V\rangle$ be the structure where:

- $\mathrm{P}(\mathcal{D})$ is the set of all p-sequents occurring in $\mathcal{D}$;

- for every $\sigma_{1}, \sigma_{2} \in \mathrm{P}(\mathcal{D}), \sigma_{1} \leq \sigma_{2}$ iff $\sigma_{2} \mapsto_{*} \sigma_{1}$;

$-\rho$ is the minimum of $\mathrm{P}(\mathcal{D})$ w.r.t. $\leq$;

- $V$ maps $\sigma \in \mathrm{P}(\mathcal{D})$ to the set $V(\sigma)=\operatorname{Lhs}(\sigma) \cap \mathcal{V}$.

One can check that $\operatorname{Mod}(\mathcal{D})$ is a model. In particular, since the root sequent of $\mathcal{D}$ is regular, there exists $\rho \in \mathrm{P}(\mathcal{D})$ such that $\sigma_{p} \mapsto_{*} \rho$, for every $\sigma_{p} \in \mathrm{P}(\mathcal{D})$, hence $\rho$ is the minimum of $\mathrm{P}(\mathcal{D})$ w.r.t. $\leq$. Moreover, by Lemma 1)(iii) and $(\mathcal{C} l 5)$, $\sigma_{1} \leq \sigma_{2}$ implies $V\left(\sigma_{1}\right) \subseteq V\left(\sigma_{2}\right)$, hence the definition of $V$ is sound. We call $\operatorname{Mod}(\mathcal{D})$ the model extracted from $\mathcal{D}$.

For every regular sequent $\sigma$ occurring in $\mathcal{D}$, let $\phi(\sigma)$ be the p-sequent immediately above $\sigma$, namely:

$$
\begin{aligned}
\phi(\sigma)=\sigma_{p} \quad \text { iff } \quad & \sigma_{p} \in \mathrm{P}(\mathcal{D}) \text { and } \sigma_{p} \mapsto_{*} \sigma \text { and } \\
& \text { for every } \sigma_{p}^{\prime} \in \mathrm{P}(\mathcal{D}), \sigma_{p} \mapsto_{*} \sigma_{p}^{\prime} \mapsto_{*} \sigma \text { implies } \sigma_{p}^{\prime}=\sigma_{p} .
\end{aligned}
$$




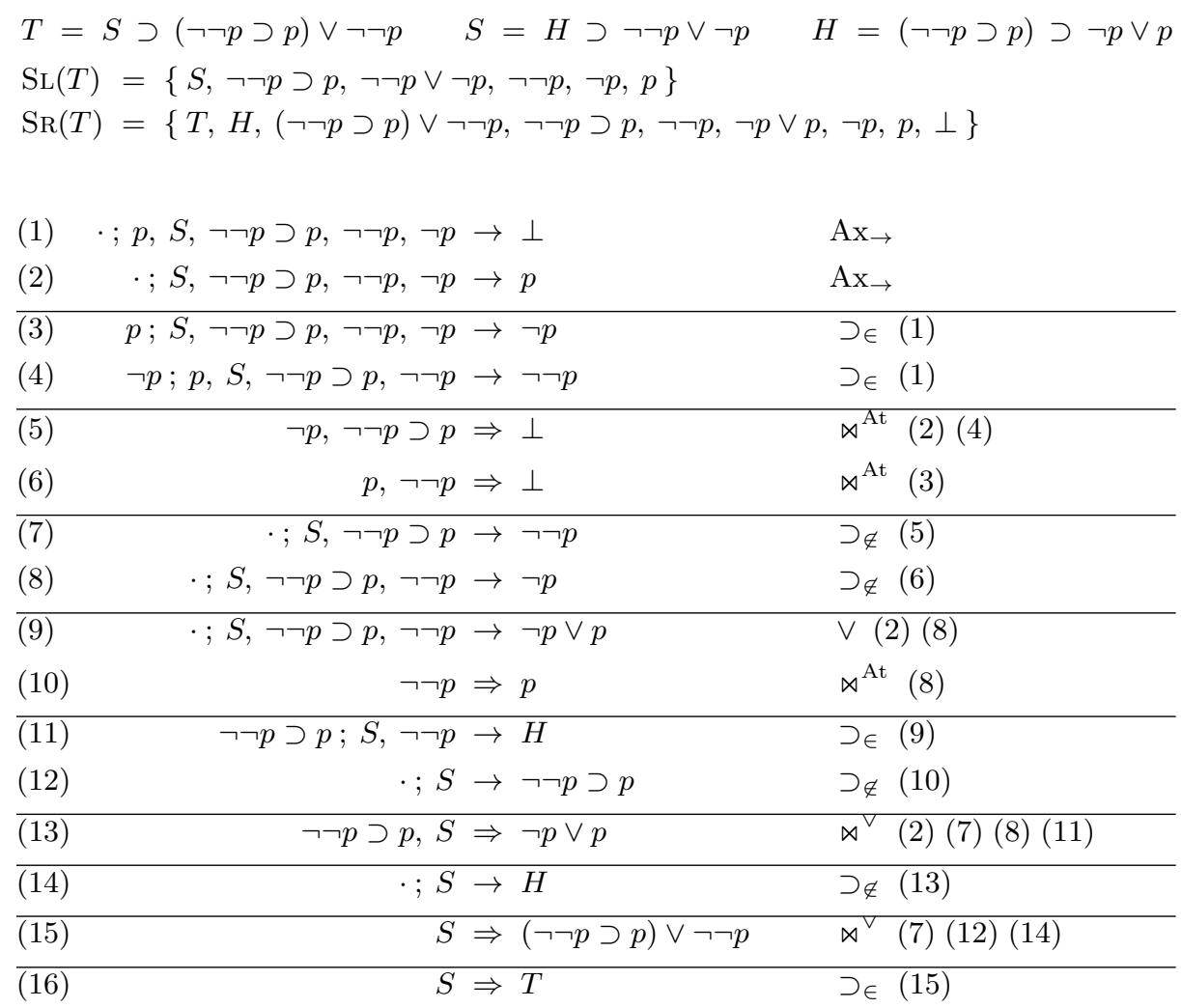

Fig. 3. The $\mathbf{F R J}(T)$-derivation $\mathcal{D}_{T}$ of $T$ and the $\operatorname{model} \operatorname{Mod}\left(\mathcal{D}_{T}\right)$.

It is easy to check that:

- p-sequents are fixed points of $\phi$, i.e., $\sigma_{p} \in \mathrm{P}(\mathcal{D})$ implies $\phi\left(\sigma_{p}\right)=\sigma_{p}$;

- $\phi$ is a surjective map from the set of regular sequents of $\mathcal{D}$ onto $\operatorname{Mod}(\mathcal{D})$;

- if $\sigma_{1}$ and $\sigma_{2}$ are regular and $\sigma_{1} \mapsto_{*} \sigma_{2}$, then $\phi\left(\sigma_{2}\right) \leq \phi\left(\sigma_{1}\right)$.

We call $\phi$ the map associated with $\mathcal{D}$. The following lemma is the key to prove the soundness properties $(\mathrm{S} 1)$ and $(\mathrm{S} 2)$ of FRJ $(G)$ stated at the beginning of Sec. 3 by $\mathrm{Sf}^{-}(C)$ we denote the set $\operatorname{Sf}(C) \backslash\{C\}$.

Lemma 2. Let $\mathcal{D}$ be an $\mathbf{F R J}(G)$-derivation of $G$, let $\operatorname{Mod}(\mathcal{D})$ be the model extracted from $\mathcal{D}$ and $\phi$ the map associated with $\mathcal{D}$. For every sequent $\sigma$ occurring in $\mathcal{D}$ :

(i) if $\sigma=\Gamma \Rightarrow C$, then $\phi(\sigma) \Vdash \Gamma$ and $\phi(\sigma) \nVdash C$; 


$$
\begin{aligned}
& K=K_{0} \supset K_{1} \quad K_{0}=\neg a \supset b \vee c \quad K_{1}=(\neg a \supset b) \vee(\neg a \supset c) \\
& \operatorname{SL}(K)=\left\{K_{0}, \neg a, a, b, c\right\} \quad \operatorname{SR}(K)=\left\{K, K_{1}, \neg a \supset b, \neg a \supset c, a, b, c, \perp\right\}
\end{aligned}
$$

$$
\begin{aligned}
\cdot ; a, b, c, K_{0}, \neg a & \rightarrow \perp & \mathrm{Ax}_{\rightarrow} \\
\cdot ; b, c, K_{0}, \neg a & \rightarrow a & \mathrm{Ax}_{\rightarrow}
\end{aligned}
$$

(3) $a ; b, c, K_{0}, \neg a \rightarrow \neg a \quad \supset \in$ (1)

$$
c, \neg a \Rightarrow b
$$$$
\bowtie^{\text {At }}
$$

$b, \neg a \Rightarrow c$ $\bowtie^{\text {At }}(2)$

$a, b, c, K_{0} \Rightarrow \perp \quad \bowtie^{\mathrm{At}}$

$$
\cdot ; c, K_{0} \rightarrow \neg a \supset b \quad \supset_{\notin} 4
$$$$
\cdot ; b, K_{0} \rightarrow \neg a \supset c
$$$$
\supset \notin \quad 5
$$

\begin{tabular}{lll}
\hline$(10)$ & $K_{0} \Rightarrow K_{1}$ & $\bowtie^{\vee}(7)(8)(9)$ \\
\hline$(11)$ & $K_{0} \Rightarrow K$ & $\supset \in(10)$
\end{tabular}

Fig. 4. The $\mathbf{F R J}(K)$-derivation $\mathcal{D}_{K}$ of $K$ and the $\operatorname{model} \operatorname{Mod}\left(\mathcal{D}_{K}\right)$.

(ii) if $\sigma=\Sigma ; \Theta \rightarrow C$, let $\sigma_{p} \in \mathrm{P}(\mathcal{D})$ such that $\sigma \mapsto \sigma_{p}$ and $\sigma_{p} \Vdash \Sigma \cap \mathrm{Sf}^{-}(C)$; then $\sigma_{p} \nVdash C$.

The proof of the lemma is outlined in [12 and is presented in full details in Appendix A. As an immediate consequence, we get:

Theorem 2. Let $\mathcal{D}$ be an $\mathbf{F R J}(G)$-derivation of $G$. Then, $\operatorname{Mod}(\mathcal{D})$ is a countermodel for $G$.

Proof. By definition, $\mathcal{D}$ is an $\mathbf{F R J}(G)$-derivation of a regular sequent $\sigma=\Gamma \Rightarrow$ $G$. Let $\phi$ be the map associated with $\mathcal{D}$. Then, $\phi(\sigma)$ is the root of $\operatorname{Mod}(\mathcal{D})$ and, by Lemma 2)(i), we have $\phi(\sigma) \nVdash G$. We conclude that $\operatorname{Mod}(\mathcal{D})$ is a countermodel for $G$.

Accordingly, if $G$ is provable in $\mathbf{F R J}(G)$, then $G$ is not valid, and this proves the Soundness of $\mathbf{F R J}(G)$ as stated in Theorem 1 .

Example 7. Let us consider the formulas $S, T$ and $K$ in examples 5 and 6 . The models $\operatorname{Mod}\left(\mathcal{D}_{S}\right), \operatorname{Mod}\left(\mathcal{D}_{T}\right), \operatorname{Mod}\left(\mathcal{D}_{K}\right)$ and the related maps $\phi$ are shown in Figs. 5, 6 and 7 respectively. The bottom world is the root and $\sigma<\sigma^{\prime}$ iff the world $\sigma$ is drawn below $\sigma^{\prime}$. For each $\sigma$, we display the set $V(\sigma)$. As an example,

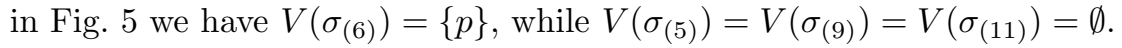



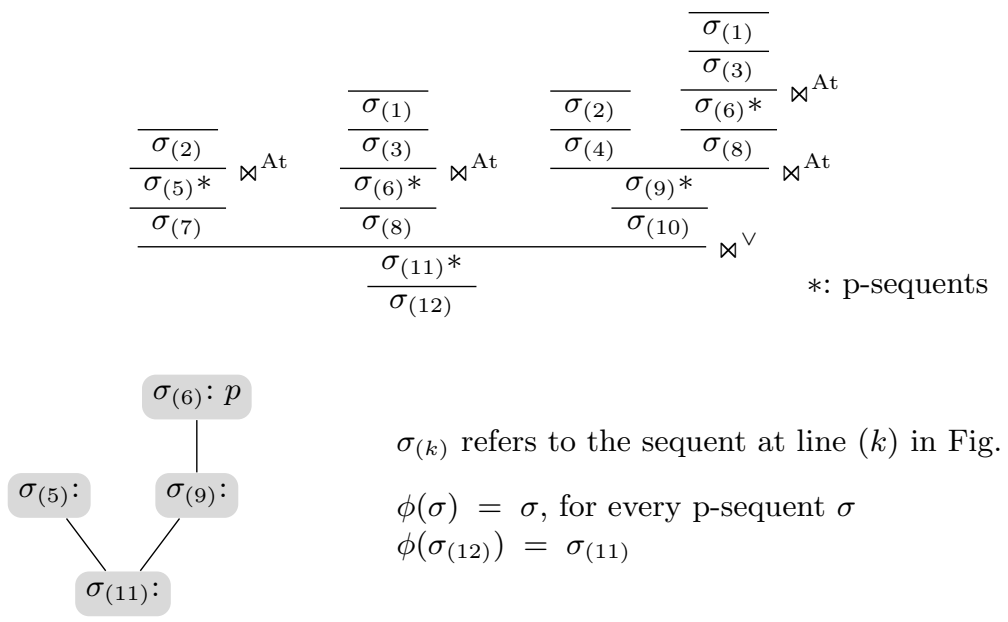

$\sigma_{(k)}$ refers to the sequent at line $(k)$ in Fig. 2

$\phi(\sigma)=\sigma$, for every p-sequent $\sigma$

$\phi(\sigma \sqrt{12})=\sigma \sqrt{11}$

Fig. 5. The model $\operatorname{Mod}\left(\mathcal{D}_{S}\right)$ (see Fig. 2).

\subsection{Termination}

To conclude the presentation of $\mathbf{F R J}(G)$, we exhibit a weight function wg on sequents of $\mathbf{F R J}(G)$ such that, after the application of a rule, the weight of sequents decreases; accordingly, the naive proof-search procedure always terminates, even if we do not implement any redundancy check. Let $\sigma_{1}$ and $\sigma_{2}$ be two $\operatorname{FRJ}(G)$-sequents and, for $k \in\{1,2\}$, let $\Gamma_{k}=\operatorname{Lhs}\left(\sigma_{k}\right)$ and $C_{k}=\operatorname{Rhs}\left(\sigma_{k}\right)$; by $\|$ II we denote the cardinality function. We show that the following properties holds:

(1) $\sigma_{1} \stackrel{\mathcal{R}}{\mapsto} \sigma_{0}$ implies $\left\|\mathcal{C l}\left(\Gamma_{2}\right) \cap \mathrm{SL}(G)\right\| \leq\left\|\mathcal{C l}\left(\Gamma_{1}\right) \cap \mathrm{SL}(G)\right\|$;

(2) $\sigma_{1} \stackrel{\supset_{\sharp}}{\mapsto} \sigma_{2}$ implies $\left\|\mathcal{C l}\left(\Gamma_{2}\right) \cap \mathrm{SL}(G)\right\|<\left\|\mathcal{C l}\left(\Gamma_{1}\right) \cap \mathrm{SL}(G)\right\|$;

(3) $\sigma_{1} \stackrel{\mathcal{R}}{\mapsto} \sigma_{2}$ and $\mathcal{R}$ is not a join rule imply $|G|-\left|C_{2}\right|<|G|-\left|C_{1}\right|$.

Let $\sigma_{1} \stackrel{\mathcal{R}}{\mapsto} \sigma_{0}$. By Lemma 1 (ii) and $(\mathcal{C} l 6)$, we have $\mathcal{C l}\left(\Gamma_{2}\right) \subseteq \mathcal{C l}\left(\Gamma_{1}\right)$, hence Point (1) holds. If $\mathcal{R}=\supset_{\notin}$, then $C_{2}=A \supset B$, where $A \in \operatorname{SL}(G)$ and $A \in \mathcal{C} l\left(\Gamma_{1}\right)$ and $A \notin \mathcal{C l}\left(\Gamma_{2}\right)$; this proves Point $(2)$. If $\mathcal{R}$ is not a join rule, then $\left|C_{2}\right|>\left|C_{1}\right|$, hence Point (3) holds. Properties (1) (3) suggest that we can define $\operatorname{wg}(\sigma)$ as the triple of non-negative integers:

$$
\begin{aligned}
& \operatorname{wg}(\sigma)=\langle\|\mathcal{C l}(\Gamma) \cap \operatorname{SL}(G)\|, \operatorname{tp}(\sigma),|G|-|C|\rangle \quad \operatorname{tp}(\sigma)=\left\{\begin{array}{l}
0 \text { if } \sigma \text { is regular } \\
1 \text { otherwise }
\end{array}\right. \\
& \Gamma=\operatorname{Lhs}(\sigma) \quad C=\operatorname{Rhs}(\sigma)
\end{aligned}
$$

Let $\prec$ be the standard lexicographic order on triples of integers; we get: 

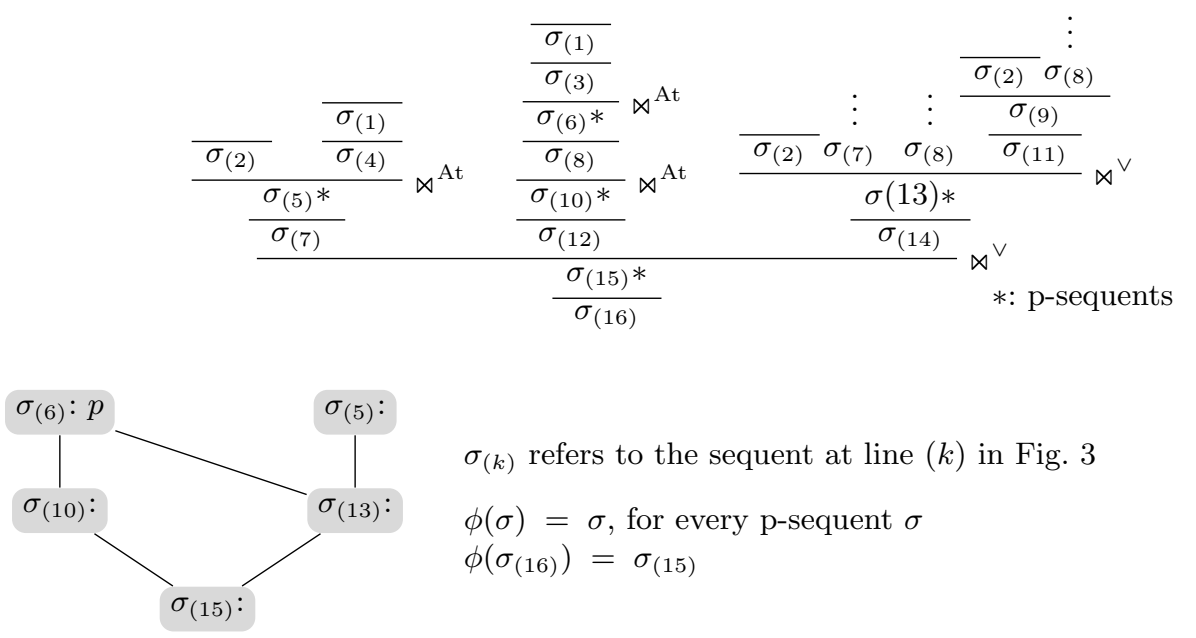

$\sigma_{(k)}$ refers to the sequent at line $(k)$ in Fig. 3

$\phi(\sigma)=\sigma$, for every p-sequent $\sigma$

$\phi(\sigma \sqrt{16})=\sigma \sqrt{15}$

Fig. 6. The model $\operatorname{Mod}\left(\mathcal{D}_{T}\right)$ (see Fig. 3).

Lemma 3. $\sigma_{1} \mapsto \sigma_{2}$ implies $\langle 0,0,0\rangle \preceq \operatorname{wg}\left(\sigma_{2}\right) \prec \operatorname{wg}\left(\sigma_{1}\right)$.

Note that the component $\operatorname{tp}(\sigma)$ accommodates the case where $\sigma_{2}$ is the conclusion of a join rule. Indeed, in this case $\sigma_{2}$ is regular and $\sigma_{1}$ is irregular, hence $\operatorname{tp}\left(\sigma_{2}\right)<\operatorname{tp}\left(\sigma_{1}\right)$.

We can exploit wg to set a bound on the height of derivations and of the extracted models. As usual a branch of an $\mathbf{F R J}(G)$-derivation is a sequence of sequents $\sigma_{1}, \ldots, \sigma_{m}$ such that $\sigma_{1} \mapsto_{0} \sigma_{2} \mapsto_{0} \cdots \mapsto_{0} \sigma_{m}$.

Lemma 4. Let $\mathcal{D}$ be an $\mathbf{F R J}(G)$-derivation, let $\mathcal{B}$ be a branch of $\mathcal{D}$ and $N=$ $|G|$. Then:

(i) the length of the branch $\mathcal{B}$ is $O\left(N^{2}\right)$;

(ii) $\mathcal{B}$ contains $N$ p-sequents at most.

Proof. Let $\mathcal{B}=\sigma_{1}, \ldots, \sigma_{m}$ where $\sigma_{1} \mapsto_{0} \sigma_{2} \mapsto_{0} \cdots \mapsto_{0} \sigma_{m}$. By Lemma 3, we have $\operatorname{wg}\left(\sigma_{m}\right) \prec \operatorname{wg}\left(\sigma_{m-1}\right) \prec \ldots \prec \operatorname{wg}\left(\sigma_{1}\right)$. For $j \in\{1, \ldots, m\}$, let $\operatorname{wg}\left(\sigma_{j}\right)=$ $\left\langle k_{j}, t_{j}, c_{j}\right\rangle$. Since $\|\mathrm{SL}(G)\| \leq N-1$, it holds that $0 \leq k_{j} \leq N-1, t_{j} \in\{0,1\}$ and $0 \leq c_{j} \leq N$, and this implies (i)

Let $\sigma_{h}=\Gamma_{h} \Rightarrow C_{h}$ and $\sigma_{l}=\Gamma_{l} \Rightarrow C_{l}$ be two distinct p-sequents of $\mathcal{B}$, where $h<l$. For $j \in\{h, l\}$, we have $\operatorname{wg}\left(\sigma_{j}\right)=\left\langle k_{j}, 0, c_{j}\right\rangle$, where $k_{j}=\left\|\mathcal{C} l\left(\Gamma_{j}\right) \cap \operatorname{SL}(G)\right\|$. Since $\sigma_{l}$ is the conclusion of a join rule, the sequent $\sigma_{l-1}$ is irregular. Accordingly, there exists $u \in\{h, \ldots, l-1\}$ such that $\sigma_{u}$ is regular and $\sigma_{u+1}$ is irregular. This means that $\sigma_{u+1}$ is obtained by an application of rule $\supset_{\notin}$, hence the branch has 


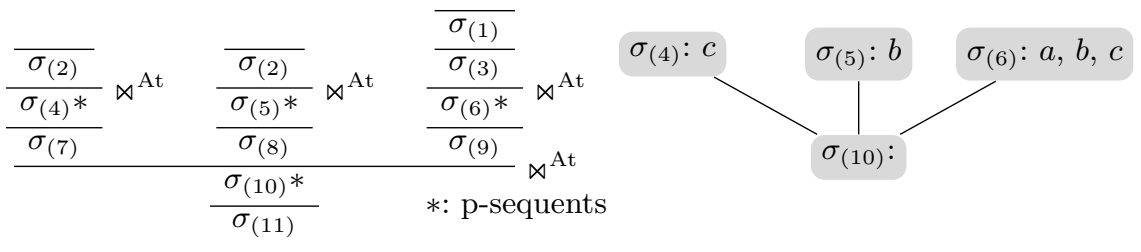

Fig. 7. The model $\operatorname{Mod}\left(\mathcal{D}_{K}\right)$ (see Fig. 4).

the following form:

$$
\begin{gathered}
\sigma_{h}=\Gamma_{h} \Rightarrow C_{h} \quad \mapsto_{*} \quad \sigma_{u}=\Gamma \Rightarrow B \quad \stackrel{\supset^{\sharp}}{\mapsto_{0}} \quad \sigma_{u+1}=\cdot ; \Theta \rightarrow A \supset B \quad \mapsto \quad \sigma_{l}=\Gamma_{l} \Rightarrow C_{l} \\
A \in \operatorname{SL}(G) \text { and } A \in \mathcal{C l}(\Gamma) \text { and } A \notin \mathcal{C l} l(\Theta)
\end{gathered}
$$

By Lemma 1(iii) and property $(\mathcal{C l 6})$ of closures, we get $\mathcal{C l}\left(\Gamma_{l}\right) \subseteq \mathcal{C l}\left(\Gamma_{h}\right), A \in$ $\mathcal{C} l\left(\Gamma_{h}\right)$ and $A \notin \mathcal{C} l\left(\Gamma_{l}\right)$. This implies $\left\|\mathcal{C} l\left(\Gamma_{l}\right) \cap \operatorname{SL}(G)\right\|<\left\|\mathcal{C} l\left(\Gamma_{h}\right) \cap \operatorname{SL}(G)\right\|$, namely $k_{l}<k_{h} \leq N$. We conclude that the branch $\mathcal{B}$ cannot contain more than $N$ distinct p-sequents.

Let $\mathcal{D}$ be an $\operatorname{FRJ}(G)$-derivation and let $\sigma$ be a sequent occurring in $\mathcal{D}$. The height of $\sigma$ (in $\mathcal{D}$ ), denoted by $\mathrm{h}(\sigma)$, is the maximum distance from $\sigma$ to an axiom sequent of $\mathcal{D}$, namely:

$$
\mathrm{h}(\sigma)=\left\{\begin{array}{l}
0 \quad \text { if } \sigma \text { is an axiom sequent } \\
1+\max \left\{\mathrm{h}\left(\sigma^{\prime}\right) \mid \sigma^{\prime} \text { occurs in } \mathcal{D} \text { and } \sigma^{\prime} \mapsto \sigma\right\} \quad \text { otherwise }
\end{array}\right.
$$

The height of $\mathcal{D}$, denoted by $\mathrm{h}(\mathcal{D})$, is the height of the root sequent of $\mathcal{D}$. Let $\mathcal{K}=\langle P, \leq, \rho, V\rangle$ be a model and let $\alpha \in P$; the height of $\alpha$ (in $\mathcal{K}$ ), denoted by $\mathrm{h}(\alpha)$, is the maximum distance from $\alpha$ to a final world of $\mathcal{K}$, namely:

$$
\mathrm{h}(\alpha)=\left\{\begin{array}{l}
0 \quad \text { if } \alpha \text { is final } \\
1+\max \{\mathrm{h}(\beta) \mid \beta \in P \text { and } \alpha<\beta\} \quad \text { otherwise }
\end{array}\right.
$$

The height of $\mathcal{K}$, denoted by $\mathrm{h}(\mathcal{K})$, is the height of $\rho$. As an immediate consequence of Lemma 4 and of the definition of $\operatorname{Mod}(\mathcal{D})$ we get an upper bound on the height of FRJ $(G)$-derivations and on the height of extracted countermodels:

Theorem 3. Let $\mathcal{D}$ be an $\mathbf{F R J}(G)$-derivation and $N=|G|$. Then:

(i) $\mathrm{h}(\mathcal{D})=O\left(N^{2}\right)$.

(ii) $\mathrm{h}(\operatorname{Mod}(\mathcal{D})) \leq N$.

In Sec 6 we present further properties on the depth of the extracted countermodels. 


\section{The proof-search procedure and saturated Databases}

The plain proof-search procedure outlined in Ex. 5 suffers from the plethora of redundant sequents generated at each iteration. To reduce the size of the database of proved sequents, we introduce the notion of subsumption.

Given two sequents $\sigma_{1}$ and $\sigma_{2}$, we say that $\sigma_{2}$ subsumes $\sigma_{1}$, and we write $\sigma_{1} \sqsubseteq \sigma_{2}$ (or $\sigma_{2} \sqsupseteq \sigma_{1}$ ) iff one of the following conditions hold:

(1) $\sigma_{1}=\Gamma_{1} \Rightarrow C$ and $\sigma_{2}=\Gamma_{2} \Rightarrow C$ and $\Gamma_{1} \subseteq \Gamma_{2}$;

(2) $\sigma_{1}=\Sigma ; \Theta_{1} \rightarrow C$ and $\sigma_{2}=\Sigma ; \Theta_{2} \rightarrow C$ and $\Theta_{1} \subseteq \Theta_{2}$.

One can easily check that the relation $\sqsubseteq$ is a partial order on the sets of $\mathbf{F R J}(G)$ sequents, since it satisfies reflexivity, antisymmetry and transitivity. By $\sigma_{1} \sqsubset \sigma_{2}$ (or $\sigma_{2} \sqsupset \sigma_{1}$ ) we mean that $\sigma_{1} \sqsubseteq \sigma_{2}$ and $\sigma_{1} \neq \sigma_{2}$.

Lemma 5. Let

$$
\frac{\sigma_{1} \cdots \sigma_{n}}{\sigma} \mathcal{R}
$$

be an instance of a rule of $\mathbf{F R J}(G)$ and let $\sigma_{1} \sqsubseteq \sigma_{1}^{\prime}, \ldots, \sigma_{n} \sqsubseteq \sigma_{n}^{\prime}$. Then,

$$
\frac{\sigma_{1}^{\prime} \cdots \sigma_{n}^{\prime}}{\sigma^{\prime}} \mathcal{R}
$$

is an instance of $\mathcal{R}$ in $\mathbf{F R J}(G)$ where $\sigma \sqsubseteq \sigma^{\prime}$.

Proof. The assertion can be proved by inspecting the rules of $\mathbf{F R J}(G)$. As an example, let us consider the case of the rule $\vee$ :

$$
\begin{array}{cc}
\sigma_{1}=\Sigma_{1} ; \Theta_{1} \rightarrow C_{1} \quad \sigma_{2}=\Sigma_{2} ; \Theta_{2} \rightarrow C_{2} \\
\sigma=\Sigma_{1}, \Sigma_{2} ; \Theta_{1} \cap \Theta_{2} \rightarrow C_{1} \vee C_{2}
\end{array} \quad \begin{aligned}
& \Sigma_{1} \subseteq \Sigma_{2} \cup \Theta_{2} \\
& \Sigma_{2} \subseteq \Sigma_{1} \cup \Theta_{1}
\end{aligned}
$$

Let $\sigma_{1}^{\prime}$ and $\sigma_{2}^{\prime}$ be such that $\sigma_{1} \sqsubseteq \sigma_{1}^{\prime}$ and $\sigma_{2} \sqsubseteq \sigma_{2}^{\prime}$. This means that $\sigma_{1}^{\prime}=$ $\Sigma_{1} ; \Theta_{1}^{\prime} \rightarrow C_{1}$ and $\sigma_{2}^{\prime}=\Sigma_{2} ; \Theta_{2}^{\prime} \rightarrow C_{2}$ with $\Theta_{1} \subseteq \Theta_{1}^{\prime}$ and $\Theta_{2} \subseteq \Theta_{2}^{\prime}$. Hence

$$
\frac{\sigma_{1}^{\prime}=\Sigma_{1} ; \Theta_{1}^{\prime} \rightarrow C_{1} \quad \sigma_{2}^{\prime}=\Sigma_{2} ; \Theta_{2}^{\prime} \rightarrow C_{2}}{\sigma^{\prime}=\Sigma_{1}, \Sigma_{2} ; \Theta_{1}^{\prime} \cap \Theta_{2}^{\prime} \rightarrow C_{1} \vee C_{2}} \vee \quad \begin{array}{ll}
\Sigma_{1} \subseteq \Sigma_{2} \cup \Theta_{2}^{\prime} \\
\Sigma_{2} \subseteq \Sigma_{1} \cup \Theta_{1}^{\prime}
\end{array}
$$

is an instance of the rule $\vee$ and, since $\Theta_{1} \cap \Theta_{2} \subseteq \Theta_{1}^{\prime} \cap \Theta_{2}^{\prime}$, we get $\sigma \sqsubseteq \sigma^{\prime}$.

In Fig. 8 we give a high-level description of the proof-search procedure FSearch for FRJ $(G)$ exploiting subsumption. We denote with $\mathrm{D}_{G}$ the database storing the sequents proved by the proof-search procedure.

Let us consider the following instance $\mathcal{R}$ of a rule of $\mathbf{F R J}(G)$

$$
\frac{\sigma_{1} \cdots \sigma_{n}}{\sigma} \mathcal{R}
$$

We say that $\mathcal{R}$ is active in $\mathrm{D}_{G}$ if $\left\{\sigma_{1}, \ldots, \sigma_{n}\right\} \subseteq \mathrm{D}_{G}$. FSearch $(G)$ maintains the database of proved sequents $\mathrm{D}_{G}$, the database $\Pi$ of generated derivations and the database $\mathcal{I}$ of sequents proved in the last performed iteration. $\mathcal{I}$ and $\Pi$ 


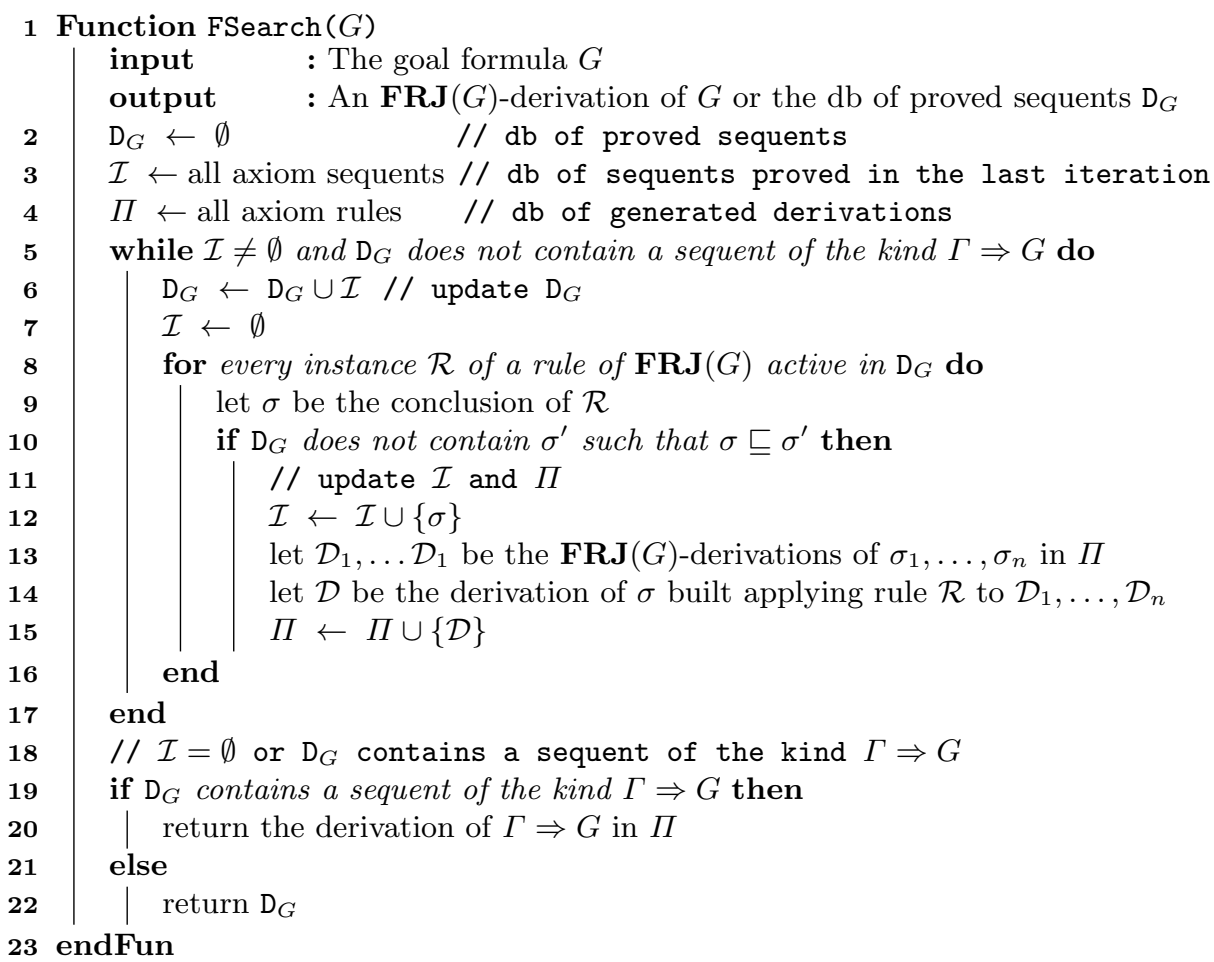

Fig. 8. The forward proof-search procedure FSearch

are initialized applying the axiom rules of $\mathbf{F R J}(G)$. The main loop (lines 5 17) performs the iterations of the proof-search procedure. At each iteration, every rule instance of $\mathbf{F R J}(G)$ active in $\mathrm{D}_{G}$ is applied; the databases $\mathcal{I}$ and $\Pi$ are updated if, as stated by condition at line 10, such an application generates a sequent not subsumed by elements in $\mathrm{D}_{G}$ (forward subsumption). We recall that applying rules $\supset_{\epsilon}, \supset_{\notin}$ and join rules we assume the restrictions (PS1) (PS4) stated in Sec. 3. The main loop terminates when either a derivation of $G$ has been built, and in this case such a derivation is returned, or no new sequent has been generated in the last iteration, and in this case the database $\mathrm{D}_{G}$ is returned. Note that, since the set of instances of the rules of $\mathbf{F R J}(G)$ is finite, FSearch $(G)$ terminates.

Now, we introduce the key notion of saturated database. Let $G$ be a formula and let $\mathrm{D}_{G}$ be a set of $\mathbf{F R J}(G)$-sequents:

(DB1) $\mathrm{D}_{G}$ is a database for $G$ iff, for every $\sigma \in \mathrm{D}_{G}, \vdash_{\mathbf{F R J}(G)} \sigma$.

(DB2) A database $\mathrm{D}_{G}$ for $G$ is saturated iff, for every $\mathbf{F R J}(G)$-sequent $\sigma$ such that $\vdash_{\mathbf{F R J}(G)} \sigma$, there exists $\sigma^{\prime} \in \mathrm{D}_{G}$ such that $\sigma \sqsubseteq \sigma^{\prime}$. 
We say that a proof-search procedure is adequate iff, for every formula $G$, the proof-search for $G$ terminates yielding either an $\mathbf{F R J}(G)$-derivation of $G$ or a saturated databases for $G$.

Theorem 4. FSearch is an adequate proof-search procedure.

Proof. Let $G$ be a formula. It is immediate to check that, if sequent $\sigma$ is added to $\mathrm{D}_{G}$ at iteration $n \geq 0$, then $\Pi$ contains a $\mathbf{F R J}(G)$-derivation of $\sigma$ of height $n$; hence $\mathrm{D}_{G}$ is a database for $G$. As a consequence, if $G$ is not provable in $\mathbf{F R J}(G)$, FSearch $(G)$ terminates returning the set $\mathrm{D}_{G}$. To prove that $\mathrm{D}_{G}$ is a saturated database, we show that, if $\mathcal{D}$ is an $\operatorname{FRJ}(G)$-derivation of $\sigma$ and $\mathrm{h}(\mathcal{D})=n$, then, at some iteration $k \in\{1, \ldots, n+1\}$ of the main loop, a sequent $\sigma^{\prime}$ such that $\sigma \sqsubseteq \sigma^{\prime}$ is added to $\mathrm{D}_{G}$. If $\mathrm{h}(\mathcal{D})=0$ then $\sigma$ is an axiom sequent, hence $\sigma$ is added to $\mathrm{D}_{G}$ at the first iteration. Let us assume that $\mathrm{h}(\mathcal{D})=h(h>0)$ and $\sigma$ is obtained by applying rule $\mathcal{R}$ to premises $\sigma_{1}, \ldots, \sigma_{m}$. By induction hypothesis there exist sequents $\sigma_{1}^{\prime}, \ldots, \sigma_{m}^{\prime}$ and integers $k_{1}, \ldots, k_{m}$ such that, for every $i \in\{0, \ldots, m\}, k_{i} \leq h+1, \sigma_{i}^{\prime}$ is added to $\mathrm{D}_{G}$ at iteration $k_{i}$ and $\sigma_{i} \sqsubseteq \sigma_{i}^{\prime}$. Let $K=\max \left\{k_{1}, \ldots, k_{n}\right\}$ and let $\sigma^{\prime}$ be the conclusion of the application of rule $\mathcal{R}$ having $\sigma_{1}^{\prime} \ldots \sigma_{n}^{\prime}$ as premises. By Lemma $5, \sigma \sqsubseteq \sigma^{\prime}$. Since $\mathcal{R}$ is active at iteration $K$, we get that, at iteration $K+1$, either $\sigma^{\prime}$ is added to $\mathrm{D}_{G}$ or $\mathrm{D}_{G}$ contains a sequent $\sigma^{\prime \prime}$ such that $\sigma \sqsubseteq \sigma^{\prime} \sqsubseteq \sigma^{\prime \prime}$. In both cases we get the assertion.

The proof-search procedure FSearch can be modified so to maintain the database of proved sequents concise according with the following definition:

(DB3) a database $\mathrm{D}_{G}$ for $G$ is compact iff, for every $\sigma \in \mathrm{D}_{G}$ there is no $\sigma^{\prime} \in \mathrm{D}_{G}$ s.t. $\sigma^{\prime} \sqsubset \sigma$.

Note that the databases in Figs. 2, 3 and 4 are compact. We show that a compact saturated database $\mathrm{D}_{G}^{*}$ for $G$ is the minimum saturated database for $G$, namely: for every saturated database $\mathrm{D}_{G}$ for $G$, it holds that $\mathrm{D}_{G}^{*} \subseteq \mathrm{D}_{G}$.

Lemma 6. Let $G$ be a formula and let $\mathrm{D}_{G}^{1}$ be a compact saturated database for $G$. For every saturated database $\mathrm{D}_{G}^{2}$ for $G, \mathrm{D}_{G}^{1} \subseteq \mathrm{D}_{G}^{2}$.

Proof. Let $\sigma_{1} \in \mathrm{D}_{G}^{1}$ and let $\mathrm{D}_{G}^{2}$ be a saturated database for $G$. By (DB1) we have $\vdash_{\mathbf{F R J}(G)} \sigma_{1}$ hence, by (DB2) there exists $\sigma_{2} \in \mathrm{D}_{G}^{2}$ such that $\sigma_{1} \sqsubseteq \sigma_{2}$. By (DB1) we have $\vdash_{\mathbf{F R J}(G)} \sigma_{2}$ hence, by (DB2), there exists $\sigma_{1}^{\prime} \in \mathrm{D}_{G}^{1}$ such that $\sigma_{2} \sqsubseteq \sigma_{1}^{\prime}$. By transitivity of $\sqsubseteq$, we get $\sigma_{1} \sqsubseteq \sigma_{1}^{\prime}$. Since $\mathrm{D}_{G}^{1}$ is compact, it is not the case that $\sigma_{1} \sqsubset \sigma_{1}^{\prime}$ (see (DB3) , hence $\sigma_{1}=\sigma_{1}^{\prime}$, which implies $\sigma_{1} \sqsubseteq \sigma_{2} \sqsubseteq \sigma_{1}$. By antisymmetry of $\sqsubseteq$ we get $\sigma_{1}=\sigma_{2}$, hence $\sigma_{1} \in \mathrm{D}_{G}^{2}$. This proves $\mathrm{D}_{G}^{1} \subseteq \mathrm{D}_{G}^{2}$.

As an immediate consequence we get:

Theorem 5. For every formula $G$, there exists a unique compact saturated database $\mathrm{D}_{G}^{*}$ for $G$, which is the minimum saturated database for $G$.

Proof. A compact saturated databases $\mathrm{D}_{G}^{*}$ for $G$ can be constructed by taking any saturated database for $G$ and removing the redundant sequents. By Lemma 6 . $\mathrm{D}_{G}^{*}$ is the minimum saturated database for $G$ and, by definition, it is unique. 
For each sequent $\sigma, \operatorname{Lhs}(\sigma) \subseteq \operatorname{SL}(G)$ and $\operatorname{Rhs}(\sigma) \in \operatorname{SR}(G)$.

$$
\begin{aligned}
& \overline{A, \Psi \Rightarrow_{g} A} \mathrm{Ax} \quad \overline{\perp, \Psi \Rightarrow_{g} C} L \perp \\
& \frac{A, B, \Psi \Rightarrow_{g} C}{A \wedge B, \Psi \Rightarrow_{g} C} L \wedge \quad \frac{\Psi \Rightarrow_{g} A \quad \Psi \Rightarrow_{g} B}{\Psi \Rightarrow_{g} A \wedge B} R \wedge \\
& \frac{A, \Psi \Rightarrow_{g} C \quad B, \Psi \Rightarrow_{g} C}{A \vee B, \Psi \Rightarrow_{g} C} L \vee \quad \frac{\Psi \rightarrow_{g} C_{k}}{\Psi \Rightarrow_{g} C_{1} \vee C_{2}} R \vee_{k} \\
& \frac{A \supset B, \Psi \rightarrow_{g} A \quad B, \Psi \Rightarrow_{g} C}{A \supset B, \Psi \Rightarrow_{g} C} L \supset \\
& \frac{\Psi \Rightarrow_{g} B}{\Psi \Rightarrow_{g} A \supset B} \supset R_{\in} \quad A \in \mathcal{C l}(\Psi) \quad \frac{A, \Psi \Rightarrow_{g} B}{\Psi \Rightarrow_{g} A \supset B} \supset R_{\notin} \quad A \notin \mathcal{C l}(\Psi) \\
& \frac{\Psi, \Psi \rightarrow_{g} A}{A x} \quad \frac{\Psi \rightarrow_{g} A \quad \Psi \rightarrow_{g} B}{\Psi \rightarrow_{g} A \wedge B} R \wedge \quad \frac{\Psi \rightarrow_{g} C_{k}}{\Psi \rightarrow_{g} C_{1} \vee C_{2}} R \vee_{k} \\
& \frac{\Psi \rightarrow_{g} B}{\Psi \rightarrow_{g} A \supset B} \supset R_{\in} \quad A \in \mathcal{C l}(\Psi) \quad \frac{A, \Psi \Rightarrow_{g} B}{\Psi \rightarrow_{g} A \supset B} \supset R_{\notin} \quad A \notin \mathcal{C l}(\Psi)
\end{aligned}
$$

Fig. 9. The calculus $\mathbf{G b u}(G)(k \in\{1,2\})$.

In the proof-search procedure of Fig. 8, we can keep compact the database of proved sequents $\mathrm{D}_{G}$ by tweaking the update instruction at line 6 as follows:

- if $\sigma$ is added, we delete from $\mathrm{D}_{G}$ all the sequents $\sigma^{\prime}$ such that $\sigma^{\prime} \sqsubset \sigma$ and all the sequents $\sigma^{\prime \prime}$ such that $\sigma^{\prime} \mapsto \sigma^{\prime \prime}$ (backward subsumption).

Clearly, the database built using this version of FSearch is compact and, using Lemma 5. we can prove that the corresponding version of FSearch is adequate.

\section{The calculus $\operatorname{Gbu}(G)$ and completeness of $\operatorname{FRJ}(G)$}

In this section we present the sequent calculus $\mathbf{G b u}(G)$ to derive the validity of a formula $G$. We show that, whenever $G$ is not provable in $\mathbf{F R J}(G)$, we can exploit a saturated database for $G$ to build a $\mathbf{G b u}(G)$-derivation of $G$, witnessing that $G$ is valid. This implies the completeness of $\mathbf{F R J}(G)$.

Let $G$ be a formula. The rules of the calculus $\mathbf{G b u}(G)$ are presented in Fig. 9 . In $\mathbf{G b u}(G)$ we have two kinds of sequents, where $\Psi \subseteq \operatorname{SL}(G)$ and $A \in \operatorname{SR}(G)$ :

- regular sequents of the form $\Psi \Rightarrow_{g} A$;

- irregular sequents of the form $\Psi \rightarrow_{g} A$.

The subscript $g$ on arrows is used to avoid confusion with $\mathbf{F R J}(G)$-sequents; for $\tau=\Psi \Rightarrow_{g} A$ or $\tau=\Psi \rightarrow_{g} A$, we set $\operatorname{Lhs}(\tau)=\Psi$ and $\operatorname{Rhs}(\tau)=A$. The 
calculus $\mathbf{G b u}(G)$ consists of two axiom rules, namely Ax and $L \perp$, and left and right rules for each connective. There are two rules to introduce an implication in the right, that is $\supset R_{\in}$ and $\supset R_{\notin}$, depending on the condition $A \in \mathcal{C l}(\Psi)$.

A $\mathbf{G b u}(G)$-sequent $\tau$ is valid (in IPL) iff the formula $(\bigwedge \operatorname{Lhs}(\tau)) \supset \operatorname{Rhs}(\tau)$ is valid (as usual, we set $\bigwedge \emptyset=\perp \supset \perp$ ). By $\vdash_{\mathbf{G b u}(G)} \tau$ we mean that $\tau$ is provable in $\mathbf{G b u}(G)$. We say that $G$ is provable in $\mathbf{G b u}(G)$, denoted by $\vdash_{\mathbf{G b u}(G)} G$, iff $\vdash_{\mathbf{G b u}(G)} \cdot \Rightarrow_{g} G$. A $\mathbf{G b u}(G)$-derivation can be trivially mapped to a derivation of the calculus G3i for IPL 23. Basically, one has to erase the distinction between regular and irregular sequents; in the translation of $\supset R_{\in}$, one has to add the antecedent $A$ to the left of the premise. Thus:

Lemma 7. If $\vdash_{\mathbf{G b u}(G)} \tau$, then $\tau$ is valid.

Accordingly, we get the Soundness of $\mathbf{G b u}(G)$ :

Theorem 6 (Soundness of $\mathbf{G b u}(G)$ ). $\vdash_{\mathbf{G b u}(G)} G$ implies $G \in$ IPL.

Differently from G3i, backward proof-search in $\mathbf{G b u}(G)$ is terminating. To show this, we introduce a weight function $\mathrm{Wg}$ on $\mathbf{G b u}(G)$-sequents such that, after the backward application of a rule, the weight of sequents decreases. Given a $\mathbf{G b u}(G)$-sequent $\tau$, the size of $\tau$, denoted by $|\tau|$, is the number of logical symbols occurring in $\tau$. Let us consider the instance

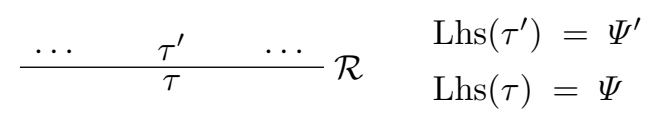

of an application of a rule of $\mathbf{G b u}(G)$, where $\tau$ is the conclusion and $\tau^{\prime}$ any of the premises. We prove the following properties:

(1) $\left\|\mathrm{SL}(G) \backslash \mathcal{C l}\left(\Psi^{\prime}\right)\right\| \leq\|\mathrm{SL}(G) \backslash \mathcal{C l}(\Psi)\|$.

(2) $\mathcal{R}=\supset R_{\notin}$ implies $\left\|\mathrm{SL}(G) \backslash \mathcal{C} l\left(\Psi^{\prime}\right)\right\|<\|\mathrm{SL}(G) \backslash \mathcal{C} l(\Psi)\|$.

(3) If $\tau^{\prime}$ is not the leftmost premise of $L \supset$, then $\left|\tau^{\prime}\right|<|\tau|$.

Point (1) follows by the fact that $\mathcal{C l}(\Psi) \subseteq \mathcal{C l}\left(\Psi^{\prime}\right)$, which implies $\mathrm{SL}(G) \backslash \mathcal{C l}\left(\Psi^{\prime}\right) \subseteq$ $\mathrm{SL}(G) \backslash \mathcal{C} l(\Psi)$. If $\mathcal{R}$ is the rule $\supset R_{\notin}$, we have $\Psi^{\prime}=\Psi \cup\{A\}$, with $A \notin \mathcal{C} l(\Psi)$. Thus, $A \in \operatorname{SL}(G) \backslash \mathcal{C l}(\Psi)$ and, since $A \in \mathcal{C} l\left(\Psi^{\prime}\right)$, we have $A \notin \mathrm{SL}(G) \backslash \mathcal{C l}\left(\Psi^{\prime}\right)$. This proves Point (2), Point (3) can be easily checked. By points (1) (3), we can define $\mathrm{Wg}(\tau)$ as the triple of non-negative integers:

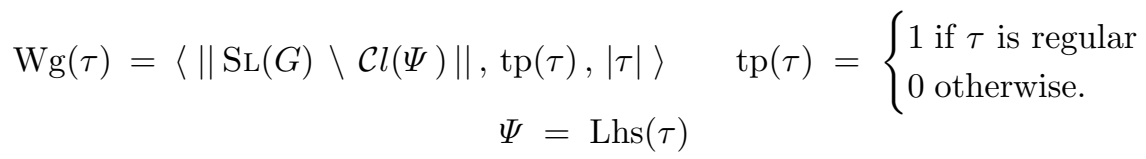

We get (where $\prec$ is lexicographic order on triples of integers):

Lemma 8. Let $\mathcal{R}$ be a rule of $\mathbf{G b u}(G)$, let $\tau$ be the conclusion of $\mathcal{R}$ and $\tau^{\prime}$ any of the premises of $\mathcal{R}$. Then $\langle 0,0,0\rangle \preceq \mathrm{Wg}\left(\tau^{\prime}\right) \prec \mathrm{Wg}(\tau)$. 
Note that the component $\operatorname{tp}(\tau)$ of $\operatorname{Wg}(\tau)$ accommodates the case where $\tau$ is the conclusion of rule $L \supset$ and $\tau^{\prime}$ the leftmost premise; in this case $\tau$ is regular and $\tau^{\prime}$ is irregular, hence $\operatorname{tp}\left(\tau^{\prime}\right)<\operatorname{tp}(\tau)$. As a consequence of Lemma 8 , and reasoning as in Lemma 4 (i), we get a bound on the height of $\mathbf{G b u}(G)$-trees:

Theorem 7. Let $\mathcal{T}$ be a $\mathbf{G b u}(G)$-tree and $\tau$ the root sequent of $\mathcal{T}$. Then, the height of $\mathcal{T}$ is $O\left(|\tau|^{2}\right)$.

Accordingly, given a $\mathbf{G b u}(G)$-sequent $\tau$, the number of $\mathbf{G b u}(G)$-trees having root sequent $\tau$ is finite, hence backward proof-search in $\mathbf{G b u}(G)$ always terminates. In contrast, we cannot set a bound on the depth of G3i-trees, since in G3i we can apply $\supset L$ an unbounded number of times.

We present the procedure BSEARCH (Backward Search) to search for a $\mathbf{G b u}(G)$ derivation of a goal formula $G$, namely a $\mathbf{G b u}(G)$-derivation of $\cdot \Rightarrow_{g} G$. BSEARCH resembles a standard backward proof-search procedure: starting from the sequent . $\Rightarrow g$, rules of $\mathbf{G b u}(G)$ are backward applied, giving precedence to the invertible rules. We recall that invertible rules can be applied in any order without affecting completeness, while the application of a non-invertible rule introduces a backtrack point: if proof-search fails, one has to backtrack and try another way. In the design of BSEARCH, we avoid backtracking by exploiting a saturated database $\mathrm{D}_{G}$ for $G$. Let:

$$
\bar{\Gamma}^{\mathrm{At}}=\mathrm{SL}(G) \cap \mathcal{V} \quad \bar{\Gamma}^{\supset}=\mathrm{SL}(G) \cap \mathcal{L}^{\supset} \quad \bar{\Gamma}=\bar{\Gamma}^{\mathrm{At}} \cup \bar{\Gamma}^{\supset}
$$

A $\mathbf{G b u}(G)$-sequent $\tau$ is critical iff one of the following conditions holds:

- $\tau=\Omega \Rightarrow_{g} D$ and $\Omega \subseteq \bar{\Gamma}$ and either $D \in \mathcal{V}^{\perp}$ or $D=C_{1} \vee C_{2}$;

- $\tau=\Omega \rightarrow_{g} C_{1} \vee C_{2}$ and $\Omega \subseteq \bar{\Gamma}$.

In backward proof-search, to a non-critical sequent we can always apply one of the rules $L \wedge, R \wedge, L \vee, \supset R_{\in}, \supset R_{\notin}$, which are the invertible rules of $\mathbf{G b u}(G)$. On the other hand, to a critical sequent we can only apply rule $L \supset$ or $R \vee_{k}$, which are not invertible. In this case, to choose the right route and avoid backtracking, we query the database $\mathrm{D}_{G}$. To extract from $\mathrm{D}_{G}$ the relevant information, we introduce the following evaluation relation $\triangleright$ :

- $\mathrm{D}_{G} \triangleright \Psi \Rightarrow_{g} C$ iff there exists $\Gamma \Rightarrow C \in \mathrm{D}_{G}$ such that $\Psi \subseteq \mathcal{C l}(\Gamma)$;

- $\mathrm{D}_{G} \triangleright \Omega \rightarrow_{g} C$ iff there exists $\Sigma ; \Theta \rightarrow C \in \mathrm{D}_{G}$ such that $\Sigma \subseteq \Omega \subseteq \Sigma \cup \Theta$.

By $\mathrm{D}_{G} \not \triangleright \sigma$, we mean that $\mathrm{D}_{G} \triangleright \sigma$ does not hold.

The recursive function BSEARCH satisfies the following specification:

- Let $\tau$ be a $\mathbf{G b u}(G)$-sequent and $\mathrm{D}_{G}$ a saturated database for $G$ such that:

(BSr1) $\mathrm{D}_{G} \not \tau$;

(BSr2) if $\tau=\Omega \rightarrow{ }_{g} C$, then $\Omega \subseteq \bar{\Gamma}$.

Then, $\operatorname{BSEARCH}\left(\sigma, \mathrm{D}_{G}\right)$ builds a $\mathbf{G b u}(G)$-derivation $\mathcal{T}$ of $\tau$.

The $\mathbf{G b u}(G)$-derivation $\mathcal{T}$ built by $\operatorname{BSEARch}\left(\tau, \mathrm{D}_{G}\right)$ is defined by cases on $\tau$. 
(B1) If $\tau$ is a $\mathbf{G b u}(G)$-axiom.

Then, $\mathcal{T}$ is the $\mathbf{G b u}(G)$-derivation only containing $\tau$.

(B2) If $\tau$ is a non-critical sequent.

Let

$$
\begin{array}{lll}
\tau_{1} \quad \cdots & \tau_{n} \\
\hline & & n \in\{1,2\} \\
& & \mathcal{R} \in\left\{L \wedge, R \wedge, L \vee, \supset R_{\in}, \supset R_{\notin}\right\}
\end{array}
$$

be any instance of a rule of $\mathbf{G b u}(G)$ having conclusion $\tau$.

For every $1 \leq j \leq n$, let $\mathcal{T}_{j}=\operatorname{BSEARCH}\left(\tau_{j}, \mathrm{D}_{G}\right)$. Then, $\mathcal{T}$ is the $\mathbf{G b u}(G)$ derivation

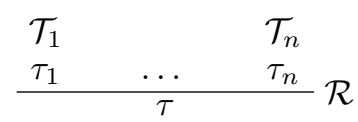

(B3) If $\tau=\Omega \rightarrow_{g} C_{1} \vee C_{2}$ (by (BSr2), $\Omega \subseteq \bar{\Gamma}$ ).

Choose any $C_{k} \in\left\{C_{1}, C_{2}\right\}$ such that $\mathrm{D}_{G} \triangleright \Omega \rightarrow{ }_{g} C_{k}$.

Let $\mathcal{T}_{k}=\operatorname{BSEARCH}\left(\Omega \rightarrow_{g} C_{k}, \mathrm{D}_{G}\right)$. Then, $\mathcal{T}$ is the $\mathbf{G b u}(G)$-derivation

$$
\begin{aligned}
& \mathcal{T}_{k} \\
& \frac{\Omega \rightarrow_{g} C_{k}}{\Omega \rightarrow_{g} C_{1} \vee C_{2}} R \vee_{k}
\end{aligned}
$$

(B4) If $\tau=\Omega \Rightarrow_{g} F$, with $F \in \mathcal{V}^{\perp}$ (note that here $\Omega \subseteq \bar{\Gamma}$ ).

Choose any $A \supset B \in \Omega$ such that $\mathrm{D}_{G} \not \supset \rightarrow_{g} A$.

Let $\Omega_{B}=(\Omega \backslash\{A \supset B\}) \cup\{B\}$ and

$$
\mathcal{T}_{1}=\operatorname{BSEARch}\left(\Omega \rightarrow_{g} A, \mathrm{D}_{G}\right) \quad \mathcal{T}_{2}=\operatorname{BSEARch}\left(\Omega_{B} \Rightarrow_{g} F, \mathrm{D}_{G}\right)
$$

Then, $\mathcal{T}$ is the $\mathbf{G b u}(G)$-derivation

$$
\frac{\mathcal{T}_{1} \rightarrow_{g} A \quad \Omega_{B} \Rightarrow_{g} F}{\Omega \Rightarrow_{g} F} L \supset
$$

(B5) Otherwise $\tau=\Omega \Rightarrow{ }_{g} C_{1} \vee C_{2}$ with $\Omega \subseteq \bar{\Gamma}$.

Let $\Upsilon=\{A \mid A \supset B \in \Omega\}$.

Choose any $Z \in \Upsilon \cup\left\{C_{1}, C_{2}\right\}$ such that $\mathrm{D}_{G} \not \triangleright \Omega \rightarrow_{g} Z$.

The derivation $\mathcal{T}$ is defined by cases on $Z$.

$-Z \in \Upsilon$.

Let $A \supset B \in \Omega$ such that $Z=A$, let $\Omega_{B}=(\Omega \backslash\{A \supset B\}) \cup\{B\}$ and

$\mathcal{T}_{1}=\operatorname{BSEarch}\left(\Omega \rightarrow_{g} A, \mathrm{D}_{G}\right) \quad \mathcal{T}_{2}=\operatorname{BSeArch}\left(\Omega_{B} \Rightarrow_{g} C_{1} \vee C_{2}, \mathrm{D}_{G}\right)$

Then, $\mathcal{T}$ is the $\mathbf{G b u}(G)$-derivation

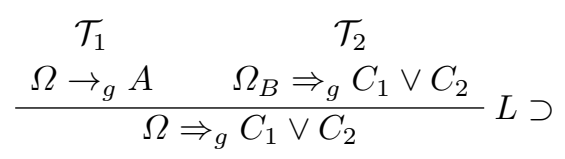


- $Z=C_{k}(k \in\{1,2\})$.

Let $\mathcal{T}_{k}=\operatorname{BSEARCH}\left(\Omega \rightarrow_{g} C_{k}, \mathrm{D}_{G}\right)$. Then, $\mathcal{T}$ is the $\mathbf{G b u}(G)$-derivation

$$
\begin{aligned}
& \mathcal{T}_{k} \\
& \frac{\Omega \rightarrow_{g} C_{k}}{\Omega \Rightarrow_{g} C_{1} \vee C_{2}} R \vee_{k}
\end{aligned}
$$

An example of computation is discussed below. The correctness of BSEARCH is proved in Theorem 8. The key issue is to show that, if cases (B1) and (B2) do not hold, then at least one of the statements described in (B3)- (B5) can be performed. We point out that in BSEARCH there are no backtrack points. We also remark that only the irregular sequents of $\mathrm{D}_{G}$ are used by BSEARCH.

Example 8. Let $E$ be the following goal formula:

$$
\begin{gathered}
E=(p \wedge A \wedge B \wedge C) \supset D \\
A=p \supset\left(q_{1} \vee q_{2}\right) \quad B=q_{1} \supset D \quad C=q_{2} \supset D \quad D=r_{1} \vee r_{2} \\
\operatorname{Lhs}(E) \cap\left(\mathcal{V} \cup \mathcal{L}^{\supset}\right)=\left\{p, q_{1}, q_{2}, r_{1}, r_{2}, A, B, C\right\} \\
\operatorname{Rhs}(E)=\left\{p, q_{1}, q_{2}, r_{1}, r_{2}, D, E\right\}
\end{gathered}
$$

Since $E$ is valid and the proof-search procedure FSearch of Fig. 8 is adequate (see Theorem 4), the call FSearch $(E)$ returns a saturated database for $E$. We can exploit it to run the procedure BSEARCH and build a $\mathbf{G b u}(E)$-derivation of $E$. We consider the compact saturated database $\mathrm{D}_{E}^{*}$ for $E$ containing the following irregular sequents $\sigma_{[1}, \ldots, \sigma_{6}$ (we omit the regular sequents since they are not used by BSEARCH):

$$
\begin{aligned}
\cdot ; q_{1}, q_{2}, r_{1}, r_{2}, A, B, C \rightarrow p & \mathrm{Ax}_{\rightarrow} \\
\cdot ; p, q_{2}, r_{1}, r_{2}, A, B, C \rightarrow q_{1} & \mathrm{Ax}_{\rightarrow} \\
\cdot ; p, q_{1}, r_{1}, r_{2}, A, B, C \rightarrow q_{2} & \mathrm{Ax}_{\rightarrow} \\
\cdot ; p, q_{1}, q_{2}, r_{2}, A, B, C \rightarrow r_{1} & \mathrm{Ax}_{\rightarrow} \\
\cdot ; p, q_{1}, q_{2}, r_{1}, A, B, C \rightarrow r_{2} & \mathrm{Ax}_{\rightarrow} \\
\cdot ; p, q_{1}, q_{2}, A, B, C \rightarrow D & \vee(4)(5)
\end{aligned}
$$

Proof-search starts from the sequent $\cdot \Rightarrow_{g} E$. Rules of $\mathbf{G b u}(E)$ are backward applied according with (B2), until the critical sequent $\tau_{1}$ is obtained:

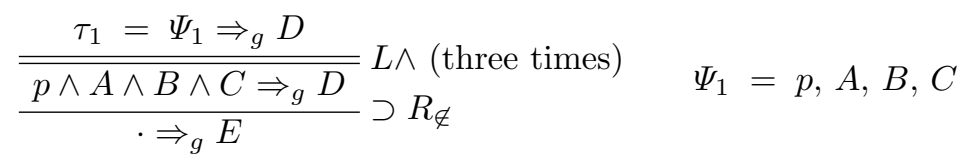

To continue the construction of the derivation, we have to bottom-up apply a rule of $\mathbf{G b u}(E)$ to the critical sequent $\tau_{1}$, and we query $\mathrm{D}_{E}^{*}$ to choose the right way. We have 5 possible choices: 
(c1) Apply rule $L \supset$ with main formula $A$; by (B5), this requires $\mathrm{D}_{E}^{*} \triangleright \Psi_{1} \rightarrow_{g} p$.

(c2) Apply rule $L \supset$ with main formula $B$; by (B5) this requires $\mathrm{D}_{E}^{*} \not \supset \Psi_{1} \rightarrow_{g} q_{1}$.

(c3) Apply rule $L \supset$ with main formula $C$; by (B5) this requires $\mathrm{D}_{E}^{*} \not \Psi_{1} \rightarrow_{g} q_{2}$.

(c4) Apply rule $R \vee_{1}$; by (B5), this requires $\mathrm{D}_{E}^{*} \not \supset \Psi_{1} \rightarrow_{g} r_{1}$.

(c5) Apply rule $R \vee_{2}$; by (B5), this requires $\mathrm{D}_{E}^{*} \not \Psi_{1} \rightarrow_{g} r_{2}$.

We have:

$$
\begin{array}{llll}
\mathrm{D}_{E}^{*} \triangleright \Psi_{1} \rightarrow_{g} q_{1} & \left(\text { see } \sigma_{\sqrt[2]{ }}\right) & \mathrm{D}_{E}^{*} \triangleright \Psi_{1} \rightarrow_{g} q_{2} & \left(\text { see } \sigma_{[3}\right) \\
\mathrm{D}_{E}^{*} \triangleright \Psi_{1} \rightarrow_{g} r_{1} & \left(\text { see } \sigma_{\sqrt[4]{4}}\right) & \mathrm{D}_{E}^{*} \triangleright \Psi_{1} \rightarrow_{g} r_{2} & \left(\text { see } \sigma_{5}\right) \\
\mathrm{D}_{E}^{*} \triangleright \Psi_{1} \rightarrow_{g} p & & &
\end{array}
$$

hence only choice (c1) can be performed. Note that, selecting any of the other options (c2) (c5), where the corresponding condition is not matched, proof-search fails. Complying with (c1) we apply rule $L \supset$ with main formula $A$ and we continue until we get the critical sequents $\tau_{2}$ and $\tau_{3}$.

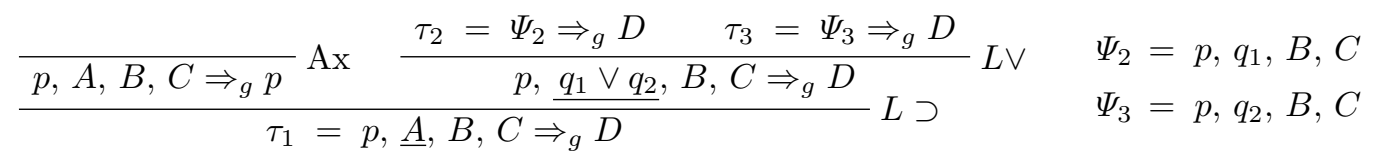

Let us consider the critical sequent $\tau_{2}$. We have 4 possible choices:

(c6) Apply rule $L \supset$ with main formula $B$; by (B5) this requires $\mathrm{D}_{E}^{*} \not \Psi_{2} \rightarrow_{g} q_{1}$.

(c7) Apply rule $L \supset$ with main formula $C$; by (B5) this requires $\mathrm{D}_{E}^{*} \triangleright \Psi_{2} \rightarrow_{g} q_{2}$.

(c8) Apply rule $R \vee_{1}$; by (B5), this requires $\mathrm{D}_{E}^{*} \not \supset \Psi_{2} \rightarrow_{g} r_{1}$.

(c9) Apply rule $R \vee_{2}$; by (B5), this requires $\mathrm{D}_{E}^{*} \not \Psi_{2} \rightarrow_{g} r_{2}$.

We note that:

$$
\begin{array}{llll}
\mathrm{D}_{E}^{*} \triangleright \Psi_{2} \rightarrow_{g} q_{2} & \left(\text { see } \sigma_{[3}\right) & \mathrm{D}_{E}^{*} \triangleright \Psi_{2} \rightarrow_{g} r_{1} & \left(\text { see } \sigma_{[4}\right) \\
\mathrm{D}_{E}^{*} \triangleright \Psi_{2} \rightarrow_{g} r_{2} & \left(\text { see } \sigma_{(55}\right) & \mathrm{D}_{E}^{*} \triangleright \Psi_{2} \rightarrow_{g} q_{1} &
\end{array}
$$

Thus, we choose (c6) and we continue until we get the critical sequents $\tau_{4}$ and $\tau_{5}$.

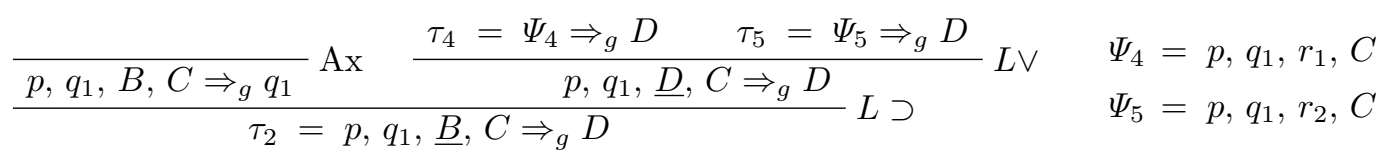

For the critical sequent $\tau_{4}$, we have 3 possible options:

(c10) Apply rule $L \supset$ with main formula $C$; by (B5) this requires $\mathrm{D}_{E}^{*} \triangleright \Psi_{4} \rightarrow_{g} q_{2}$.

(c11) Apply rule $R \vee_{1}$; by (B5), this requires $\mathrm{D}_{E}^{*} \not \supset \Psi_{4} \rightarrow_{g} r_{1}$.

(c12) Apply rule $R \vee_{2}$; by (B5), this requires $\mathrm{D}_{E}^{*} \not \Psi_{4} \rightarrow_{g} r_{2}$. 
We have

$$
\mathrm{D}_{E}^{*} \triangleright \Psi_{4} \rightarrow_{g} q_{2} \quad\left(\text { see } \sigma_{[3}\right) \quad \mathrm{D}_{E}^{*} \triangleright \Psi_{4} \rightarrow_{g} r_{2} \quad\left(\text { see } \sigma_{\text {5 }}\right) \quad \mathrm{D}_{E}^{*} \triangleright \Psi_{4} \rightarrow_{g} r_{1}
$$

Thus, we select (c11) and we get

$$
\frac{\overline{p, q_{1}, r_{1}, C \Rightarrow_{g} r_{1}} \mathrm{Ax}}{\tau_{4}=p, q_{1}, r_{1}, C \Rightarrow_{g} D} R \vee_{1}
$$

The $\mathbf{G b u}(E)$-derivations of the sequents $\tau_{5}$ and $\tau_{3}$ have a similar construction:

$$
\frac{\overline{p, q_{1}, r_{2}, C \Rightarrow_{g} r_{2}} \mathrm{Ax}}{\tau_{5}=p, q_{1}, r_{2}, C \Rightarrow_{g} D} R \vee_{2}
$$

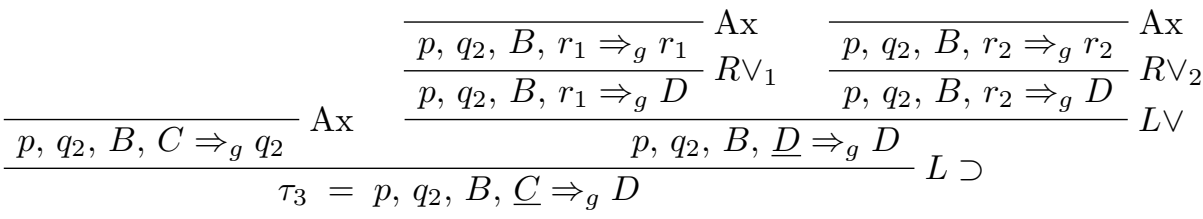

and this completes the definition of the $\mathbf{G b u}(E)$-derivation of $E$.

Now we prove the correctness of BSEARCH. We start by showing some properties of the evaluation relation.

Lemma 9. Let $G$ be a formula, let $\mathrm{D}_{G}$ be a saturated database for $G, \Psi \subseteq \operatorname{SL}(G)$ and $\Omega \subseteq \bar{\Gamma}$.

(i) If $\mathrm{D}_{G} \triangleright A, B, \Psi \Rightarrow_{g} C$, then $\mathrm{D}_{G} \triangleright A \wedge B, \Psi \Rightarrow_{g} C$.

(ii) If $C_{1} \wedge C_{2} \in \operatorname{SR}(G)$ and $\mathrm{D}_{G} \triangleright \Psi \Rightarrow_{g} C_{k}$ with $k \in\{1,2\}$ then $\mathrm{D}_{G} \triangleright \Psi \Rightarrow_{g}$ $C_{1} \wedge C_{2}$.

(iii) If $A_{1} \vee A_{2} \in \mathrm{SL}(G)$ and $\mathrm{D}_{G} \triangleright A_{k}, \Psi \Rightarrow_{g} C$ with $k \in\{1,2\}$, then $\mathrm{D}_{G} \triangleright A_{1} \vee$ $A_{2}, \Psi \Rightarrow_{g} C$.

(iv) If $A \supset B \in \mathrm{SL}(G)$ and $\mathrm{D}_{G} \triangleright B, \Psi \Rightarrow_{g} C$, then $\mathrm{D}_{G} \triangleright A \supset B, \Psi \Rightarrow_{g} C$.

(v) If $A \supset B \in \mathrm{SR}(G)$ and $\mathrm{D}_{G} \triangleright \Psi \Rightarrow_{g} B$, with $A \in \mathcal{C l}(\Psi)$, then $\mathrm{D}_{G} \triangleright \Psi \Rightarrow_{g}$ $A \supset B$.

(vi) If $A \supset B \in \operatorname{SR}(G)$ and $\mathrm{D}_{G} \triangleright A, \Psi \Rightarrow_{g} B$ with $A \notin \mathcal{C l}(\Psi)$, then $\mathrm{D}_{G} \triangleright \Psi \Rightarrow_{g}$ $A \supset B$.

(vii) If $C_{1} \wedge C_{2} \in \mathrm{SR}(G)$ and $\mathrm{D}_{G} \triangleright \Omega \rightarrow_{g} C_{k}$ with $k \in\{1,2\}$, then $\mathrm{D}_{G} \triangleright \Omega \rightarrow_{g}$ $C_{1} \wedge C_{2}$.

(viii) If $A \supset B \in \operatorname{SR}(G)$ and $\mathrm{D}_{G} \triangleright \Omega \rightarrow_{g} B$ with $A \in \mathcal{C l}(\Omega)$, then $\mathrm{D}_{G} \triangleright \Omega \rightarrow_{g}$ $A \supset B$.

(ix) If $\mathrm{D}_{G} \triangleright A, \Omega \Rightarrow_{g} B$ with $A \notin \mathcal{C} l(\Omega)$, then $\mathrm{D}_{G} \triangleright \Omega \rightarrow_{g} A \supset B$.

Proof. We only detail some representative cases.

Proof of (i) Let $\mathrm{D}_{G} \triangleright A, B, \Psi \Rightarrow_{g} C$. Then, there exists $\Gamma$ such that:

$-\Gamma \Rightarrow C \in \mathrm{D}_{G}$

$-\Psi \cup\{A, B\} \subseteq \mathcal{C} l(\Gamma)$.

Since $A \wedge B \in \mathcal{C} l(\Gamma)$, we have $\Psi \cup\{A \wedge B\} \subseteq \mathcal{C} l(\Gamma)$, hence $\mathrm{D}_{G} \triangleright A \wedge B, \Psi \Rightarrow_{g} C$. 
Proof of (ii) Let $C_{1} \wedge C_{2} \in \mathrm{SR}(G)$ and let us assume $\mathrm{D}_{G} \triangleright \Psi \Rightarrow_{g} C_{k}$, with $k \in\{1,2\}$. Then, there exists $\Gamma$ such that:

$-\Gamma \Rightarrow C_{k} \in \mathrm{D}_{G}$;

$-\Psi \subseteq \mathcal{C l}(\Gamma)$.

By (DB1), $\vdash_{\mathbf{F R J}(G)} \Gamma \Rightarrow C_{k}$, hence $\vdash_{\mathbf{F R J}(G)} \Gamma \Rightarrow C_{1} \wedge C_{2}$. By (DB2), $\mathrm{D}_{G}$ contains a sequent $\Gamma^{\prime} \Rightarrow C_{1} \wedge C_{2}$ such that $\Gamma \subseteq \Gamma^{\prime}$. By $(\mathcal{C l} l 4), \mathcal{C l}(\Gamma) \subseteq \mathcal{C l}\left(\Gamma^{\prime}\right)$, which implies $\Psi \subseteq \mathcal{C l}\left(\Gamma^{\prime}\right)$, hence $\mathrm{D}_{G} \triangleright \Psi \Rightarrow_{g} C_{1} \wedge C_{2}$.

Proof of (v) Let $A \supset B \in \operatorname{SR}(G)$ and let us assume $\mathrm{D}_{G} \triangleright \Psi \Rightarrow_{g} B$, with $A \in \mathcal{C l}(\Psi)$. Then, there exists $\Gamma$ such that:

$-\Gamma \Rightarrow B \in \mathrm{D}_{G}$

$-\Psi \subseteq \mathcal{C l}(\Gamma)$.

By (DB1) $\vdash_{\text {FRJ }(G)} \Gamma \Rightarrow B$. By $(\mathcal{C l} l 6)$ we get $\mathcal{C l}(\Psi) \subseteq \mathcal{C l}(\Gamma)$, hence $A \in \mathcal{C l}(\Gamma)$; this implies $\vdash_{\text {FRJ }(G)} \Gamma \Rightarrow A \supset B$. Reasoning as in the proof of case (ii), we get $\mathrm{D}_{G} \triangleright \Psi \Rightarrow_{g} A \supset B$.

Proof of (vi) Let $A \supset B \in \mathrm{SR}(G)$ and let us assume $\mathrm{D}_{G} \triangleright A, \Psi \Rightarrow_{g} B$, with $A \notin \mathcal{C l}(\Psi)$. Then, there exists $\Gamma$ such that:

$-\Gamma \Rightarrow B \in \mathrm{D}_{G}$

$-\Psi \cup\{A\} \subseteq \mathcal{C} l(\Gamma)$.

By (DB1), $\vdash_{\text {FRJ }(G)} \Gamma \Rightarrow B$, hence $\vdash_{\text {FRJ }(G)} \Gamma \Rightarrow A \supset B$. Reasoning as in the proof of case (ii), we get $\mathrm{D}_{G} \triangleright \Psi \Rightarrow_{g} A \supset B$.

Proof of (viii) Let us assume $\mathrm{D}_{G} \triangleright \Omega \rightarrow_{g} B$ and $A \in \mathcal{C} l(\Omega)$. Then, there exist $\Sigma$ and $\Theta$ such that:

$-\Sigma ; \Theta \rightarrow B \in \mathrm{D}_{G}$

$-\Sigma \subseteq \Omega \subseteq \Sigma \cup \Theta$.

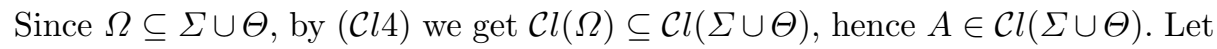
$\Lambda$ be a minimum (possibly empty) subset of $\Theta$ such that $A \in \mathcal{C} l(\Sigma \cup \Lambda)$. Since $A \in \mathcal{C l}(\Omega)$ and $\Sigma \subseteq \Omega \subseteq \Sigma \cup \Theta$, we can choose $\Lambda$ so that $\Sigma \cup \Lambda \subseteq \Omega$. By (DB1) $\vdash_{\mathbf{F R J}(G)} \Sigma ; \Theta \rightarrow B$, hence we can build the $\mathbf{F R J}(G)$-derivation:

$$
\begin{array}{cl}
\vdots & \Theta_{1}=\Theta \backslash \Lambda \\
\frac{\Lambda \subseteq \Theta \text { and } A \in \mathcal{C l}(\Sigma \cup \Lambda)}{\Theta} \overbrace{\Lambda, \Theta_{1}}^{\Theta} \rightarrow B \\
\Sigma, \Lambda ; \Theta_{1} \rightarrow A \supset B & \Lambda^{\prime} \subsetneq \Lambda \text { implies } A \notin \mathcal{C l}\left(\Sigma \cup \Lambda^{\prime}\right)
\end{array}
$$

Note that the above derivation matches (PS1), By (DB2), $\mathrm{D}_{G}$ contains a sequent $\Sigma, \Lambda ; \Theta^{\prime} \rightarrow A \supset B$ such that $\Theta_{1} \subseteq \Theta^{\prime}$. Since $\Omega \subseteq \Sigma \cup \Theta$ and $\Sigma \cup \Theta=\Sigma \cup \Lambda \cup \Theta_{1}$, we get $\Omega \subseteq \Sigma \cup \Lambda \cup \Theta^{\prime}$. Thus $\Sigma \cup \Lambda \subseteq \Omega \subseteq \Sigma \cup \Lambda \cup \Theta^{\prime}$, and this proves that $\mathrm{D}_{G} \triangleright \Omega \rightarrow_{g} A \supset B$. 
Proof of (ix) Let us assume $\mathrm{D}_{G} \triangleright A, \Omega \Rightarrow_{g} B$ and $A \notin \mathcal{C l}(\Omega)$. Then, there exists $\Gamma$ such that:

$-\Gamma \Rightarrow B \in \mathrm{D}_{G}$

$-\Omega \cup\{A\} \subseteq \mathcal{C l}(\Gamma)$.

Let $\Theta$ be a maximal subset of $\mathcal{C l}(\Gamma) \cap \bar{\Gamma}$ such that $A \notin \mathcal{C l}(\Theta)$; since $\Omega \subseteq \mathcal{C l}(\Gamma) \cap \bar{\Gamma}$ and $A \notin \mathcal{C l}(\Omega)$, we can choose $\Theta$ so that $\Omega \subseteq \Theta$. By (DB1), $\vdash_{\text {FRJ }(G)} \Gamma \Rightarrow B$, hence we can build the following $\mathbf{F R J}(G)$-derivation:

$$
\begin{array}{cl}
\vdots & \Omega \subseteq \Theta \subseteq \mathcal{C l}(\Gamma) \cap \bar{\Gamma} \\
\frac{\Gamma \Rightarrow B}{\cdot ; \Theta \rightarrow A \supset B} \supset_{\notin} & A \in \mathcal{C l}(\Gamma) \backslash \mathcal{C} l(\Theta) \\
& \Theta \subsetneq \Theta^{\prime} \subseteq \mathcal{C} l(\Gamma) \cap \bar{\Gamma} \text { implies } A \in \mathcal{C l}\left(\Theta^{\prime}\right)
\end{array}
$$

Note that the above derivation matches (PS2) By (DB2), $\mathrm{D}_{G}$ contains a sequent ·; $\Theta^{\prime} \rightarrow A \supset B$, where $\Theta \subseteq \Theta^{\prime}$; since $\Omega \subseteq \Theta^{\prime}$, we get $\mathrm{D}_{G} \triangleright \Omega \rightarrow_{g} A \supset B$.

Lemma 10. Let $G$ be a formula, let $\mathrm{D}_{G}$ be a saturated database for $G$ and $\Omega \subseteq \bar{\Gamma}$. If $\mathrm{D}_{G} \triangleright \Omega \rightarrow_{g} C_{1}$ and $\mathrm{D}_{G} \triangleright \Omega \rightarrow_{g} C_{2}$, then $\mathrm{D}_{G} \triangleright \Omega \rightarrow_{g} C_{1} \vee C_{2}$.

Proof. Let us assume $\mathrm{D}_{G} \triangleright \Omega \rightarrow_{g} C_{1}$ and $\mathrm{D}_{G} \triangleright \Omega \rightarrow_{g} C_{2}$. For $k \in\{1,2\}$, there exist $\Sigma_{k}$ and $\Theta_{k}$ be such that:

$-\Sigma_{k} ; \Theta_{k} \rightarrow C_{k} \in \mathrm{D}_{G}$

$-\Sigma_{k} \subseteq \Omega \subseteq \Sigma_{k} \cup \Theta_{k}$.

By (DB1) $\vdash_{\text {FRJ }(G)} \Sigma_{k} ; \Theta_{k} \rightarrow C_{k}$. Note that $\Sigma_{1} \subseteq \Sigma_{2} \cup \Theta_{2}$ and $\Sigma_{2} \subseteq \Sigma_{1} \cup \Theta_{1}$, hence we can build the following $\mathbf{F R J}(G)$-derivation:

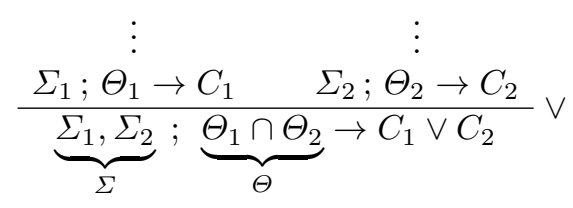

By (DB2), $\mathrm{D}_{G}$ contains a sequent $\Sigma ; \Theta^{\prime} \rightarrow C_{1} \vee C_{2}$ such that $\Theta \subseteq \Theta^{\prime}$. One can easily check that $\Sigma \subseteq \Omega$ and $\Omega \subseteq \Sigma \cup \Theta$, which implies $\Omega \subseteq \Sigma \cup \Theta^{\prime}$. We conclude $\mathrm{D}_{G} \triangleright \Omega \rightarrow_{g} C_{1} \vee C_{2}$.

Lemma 11. Let $G$ be a formula and let $\mathrm{D}_{G}$ be a saturated database for $G$. Let $\tau=\Omega \Rightarrow_{g} F$ be such that $\Omega \subseteq \bar{\Gamma}$ and $F \in \mathcal{V}^{\perp}$, and let us assume that:

(i) $F \notin \Omega$;

(ii) for every $A \supset B \in \Omega, \mathrm{D}_{G} \triangleright \Omega \rightarrow_{g} A$.

Then, $\mathrm{D}_{G} \triangleright \tau$. 
Proof. Let $\Omega=\Omega^{\mathrm{At}} \cup \Omega^{\supset}$. Let us assume that $\Omega^{\supset}$ is empty, namely $\Omega=\Omega^{\mathrm{At}}$, and let $\Gamma^{\prime}=\bar{\Gamma}^{\text {At }} \backslash\{F\}$. Then, $\sigma^{\prime}=\Gamma^{\prime} \Rightarrow F$ is an axiom sequent of $\mathbf{F R J}(G)$ hence, by (DB2) $\mathrm{D}_{G}$ contains a sequent $\sigma^{\prime \prime}=\Gamma \Rightarrow F$ with $\Gamma^{\prime} \subseteq \Gamma$. Since $\Omega^{\mathrm{At}} \subseteq \Gamma$, by $[(\mathcal{C} l 3)]$ we get $\Omega^{\mathrm{At}} \subseteq \mathcal{C} l(\Gamma)$, hence $\mathrm{D}_{G} \triangleright \tau$.

Let $\Omega^{\supset}$ be nonempty and let $\Omega^{\supset}=\left\{A_{1} \supset B_{1}, \ldots, A_{n} \supset B_{n}\right\}(n \geq 1)$. For every $k \in\{1, \ldots, n\}$, since $\mathrm{D}_{G} \triangleright \Omega \rightarrow_{g} A_{k}$, there are $\Sigma_{k}$ and $\Theta_{k}$ such that:

$-\Sigma_{k} ; \Theta_{k} \rightarrow A_{k} \in \mathrm{D}_{G}$

$-\Sigma_{k} \subseteq \Omega \subseteq \Sigma_{k} \cup \Theta_{k}$.

By (DB1) $\vdash_{\text {FRJ }(G)} \Sigma_{k} ; \Theta_{k} \rightarrow A_{k}$. One can easily check that, for $i \neq j$, it holds that $\Sigma_{i} \subseteq \Sigma_{j} \cup \Theta_{j}$. For $k \in\{1, \ldots, n\}$, let $\Sigma_{k}=\Sigma_{k}^{\text {At }} \cup \Sigma_{k}^{\supset}$ and $\Theta_{k}=\Theta_{k}^{\text {At }} \cup \Theta_{k}^{\supset}$. We can build the following $\mathbf{F R J}(G)$-derivation $\left(\Sigma^{\mathrm{At}}, \Sigma^{\supset}, \Theta^{\mathrm{At}}, \Theta^{\supset}\right.$ are defined as in Fig. 11:

$$
\begin{gathered}
\vdots \vdots \\
\frac{\Sigma_{1} ; \Theta_{1} \rightarrow A_{1} \ldots \Sigma_{n} ; \Theta_{n} \rightarrow A_{n}}{\Gamma \Rightarrow F} \bowtie^{A t} \\
\Gamma=\Sigma^{\text {At }} \cup \Sigma^{\supset} \cup\left(\Theta^{\text {At }} \backslash\{F\}\right) \cup \Theta^{\supset}
\end{gathered}
$$

Note that the above derivation matches the restriction (PS3) on the application of $\bowtie^{\mathrm{At}}$ stated in Sect. 3. By (DB2), $\mathrm{D}_{G}$ contains a sequent $\Gamma^{\prime} \Rightarrow F$, with $\Gamma \subseteq \Gamma^{\prime}$; by $(\mathcal{C l}), \mathcal{C l}(\Gamma) \subseteq \mathcal{C} l\left(\Gamma^{\prime}\right)$. One can easily check that $\Omega \subseteq \Gamma$ hence, by $(\mathcal{C} l 3)$, $\Omega \subseteq \mathcal{C l}(\Gamma)$. Thus $\Omega \subseteq \mathcal{C} l\left(\Gamma^{\prime}\right)$, which implies $\mathrm{D}_{G} \triangleright \Omega \rightarrow_{g} F$.

In a similar way we can prove that:

Lemma 12. Let $G$ be a formula and let $\mathrm{D}_{G}$ be a saturated database for $G$. Let $\tau=\Omega \Rightarrow{ }_{g} C_{1} \vee C_{2}$ be such that $\Omega \subseteq \bar{\Gamma}$, and let us assume that:

(i) for every $A \supset B \in \Omega, \mathrm{D}_{G} \triangleright \Omega \rightarrow_{g} A$.

(ii) $\mathrm{D}_{G} \triangleright \Omega \rightarrow_{g} C_{1}$ and $\mathrm{D}_{G} \triangleright \Omega \rightarrow_{g} C_{2}$.

Then, $\mathrm{D}_{G} \triangleright \tau$.

We prove the correctness of BSEARCH.

Theorem 8 (Correctness of BSEARCH). Let $G$ be a formula, let $\tau$ be a $\mathbf{G b u}(G)$-sequent and let $\mathrm{D}_{G}$ be a saturated database for $G$ satisfying (BSr1) and (BSr2), Then, $\operatorname{BSEARCH}\left(\tau, \mathrm{D}_{G}\right)$ computes a $\mathbf{G b u}(G)$-derivation of $\tau$.

Proof. We prove the assertion by induction on $\mathrm{Wg}(\tau)$. Note that, whenever we perform a recursive call $\operatorname{BSEARCH}\left(\tau^{\prime}, \mathrm{D}_{G}\right)$, it holds that $\mathrm{Wg}\left(\tau^{\prime}\right) \prec \mathrm{Wg}(\tau)$. Thus, by the induction hypothesis, we can assume that:

$(\dagger)$ every recursive call BSEARCH$\left(\tau^{\prime}, \mathrm{D}_{G}\right)$ yields a $\mathbf{G b u}(G)$-derivation of $\tau^{\prime}$, provided that $\tau^{\prime}$ and $\mathrm{D}_{G}$ satisfy assumptions (BSr1) and (BSr2) 
We have to show that, in each of the cases (B1) (B5), a $\mathbf{G b u}(G)$-derivation $\mathcal{T}$ of $\tau$ is built. Case (B1) is immediate. Let us consider Case (B2) namely $\tau$ is non-critical. One can easily check that at least an application of a rule $\mathcal{R}$, as displayed in (B2) is possible. We have to show that each premise $\tau_{j}$ of the selected rule $\mathcal{R}$ satisfies assumptions (BSr1) and (BSr2) Le us consider assumption (BSr2) if $\tau_{j}$ is regular, then (BSr2) trivially holds. If $\tau_{j}$ is irregular, then, by inspecting the rules of $\mathbf{G b u}(G)$, one can check that $\operatorname{Lhs}\left(\tau_{j}\right)=\operatorname{Lhs}(\tau)$; thus, since $\tau$ satisfies (BSr2), $\tau_{j}$ satisfies (BSr2) as well. The validity of (BSr1) follows by Lemma 9. For instance, let us assume that $\mathcal{R}=L \vee$ and that the selected application is

$$
\frac{\tau_{1}=A, \Psi \Rightarrow_{g} C \quad \tau_{2}=B, \Psi \Rightarrow_{g} C}{\tau=A \vee B, \Psi \Rightarrow_{g} C} L \vee
$$

Since $\tau$ satisfies (BSr1), we have $\mathrm{D}_{G} \triangleright \tau$; by Lemma $9($ iii), it follows that both $\mathrm{D}_{G} \triangleright \tau_{1}$ and $\mathrm{D}_{G} \not \supset \tau_{2}$, hence both $\tau_{1}$ and $\tau_{2}$ satisfy (BSr1) By (†), for every $1 \leq j \leq n, \mathcal{T}_{j}$ is a $\mathbf{G b u}(G)$-derivation of $\tau_{j}$, hence $\mathcal{T}$ is a $\mathbf{G b u}(G)$-derivation of $\tau$.

Let $\tau$ match Case (B3). Then, $\tau=\Omega \rightarrow_{g} C_{1} \vee C_{2}$ and $\Omega \subseteq \bar{\Gamma}$. Let $\tau_{k}=\Omega \rightarrow_{g}$ $C_{k}$, with $k \in\{1,2\}$. We have to guarantee that there exists $k \in\{1,2\}$ such that $\mathrm{D}_{G} \triangleright \tau_{k}$. If both $\mathrm{D}_{G} \triangleright \tau_{1}$ and $\mathrm{D}_{G} \triangleright \tau_{2}$ hold, by Lemma 10 we would conclude $\mathrm{D}_{G} \triangleright \tau$, in contradiction with the fact that $\tau$ and $\mathrm{D}_{G}$ satisfy (BSr1) Thus, we can choose $k$ such that $\mathrm{D}_{G} \not \tau_{k}$. Since $\tau_{k}$ and $\mathrm{D}_{G}$ satisfy assumptions (BSr1) and (BSr2), by $(\dagger) \mathcal{T}_{k}$ is a $\mathbf{G b u}(G)$-derivation of $\tau_{k}$, hence $\mathcal{T}$ is a $\mathbf{G b u}(G)$-derivation of $\tau$.

Let $\tau$ match Case (B4) Since Case (B1) does not hold, $\tau$ is not a $\mathbf{G b u}(G)$ axiom, hence $F \notin \Omega$. Let us assume that for every $A \supset B \in \Omega$, we have $\mathrm{D}_{G} \triangleright$ $\Omega \rightarrow_{g} A$. By Lemma 11, it would follow $\mathrm{D}_{G} \triangleright \tau$, against the assumption (BSr1) of the lemma. Thus, we can pick $A \supset B \in \Omega$ such that $\mathrm{D}_{G} \not \triangleright \Omega \rightarrow_{g} A$. By $(\dagger) \mid \mathcal{T}_{1}$ is a $\mathbf{G b u}(G)$-derivation of $\tau_{1}=\Omega \rightarrow_{g} A$. By (†) and Lemma g(iv), $\mathcal{T}_{2}$ is a $\mathbf{G b u}(G)$ derivation of $\tau_{2}=\Omega_{B} \Rightarrow_{g} F$. We conclude that $\mathcal{T}$ is a $\mathbf{G b u}(G)$-derivation of $\tau=\Omega \Rightarrow_{g} F$.

We observe that, if $\tau$ does not match any of the conditions in cases (B1). (B4), then $\tau$ has the form stated in Case (B5) Case (B5) follows along the lines of Case (B4), exploiting Lemma 12.

By the correctness of BSEarch, it follows that $\mathbf{G b u}(G)$ and $\mathbf{F R J}(G)$ are dual calculi, in the sense that provability in $\mathbf{G b u}(G)$ is the complement of provability in $\mathbf{F R J}(G)$ :

Theorem 9. $\vdash_{\mathbf{G b u}(G)} G$ iff $\nvdash_{\mathbf{F R J}(G)} G$.

Proof. If $\vdash_{\mathbf{G b u}(G)} G$, by the Soundness of $\mathbf{G b u}(G)$ (Theorem 6 ) we get $G \in$ IPL; by the Soundness of $\mathbf{F R J}(G)$ (Theorem 1 ) we conclude $\nvdash_{\text {FRJ }(G)} G$.

Conversely, let $\nvdash_{\mathbf{F R J}(G)} G$, let $\mathrm{D}_{G}$ be a saturated database for $G$ and let $\tau$ be the $\mathbf{G b u}(G)$-sequent $\cdot \Rightarrow_{g} G$. Note that $\mathrm{D}_{G}$ does not contain any regular sequent of the kind $\sigma=\Gamma \Rightarrow G$; otherwise, by (DB1), we would get $\vdash_{\mathbf{F R J}(G)} \sigma$, contradicting the assumption that $G$ is not provable in $\mathbf{F R J}(G)$. This implies $\mathrm{D}_{G} \triangleright \tau$, 
hence $\tau$ and $\mathrm{D}_{G}$ satisfy the assumptions (BSr1) and (BSr2) of BSEARCH. By the correctness of BSEARCH (Theorem 8), $\operatorname{BSEARCH}\left(\tau, \mathrm{D}_{G}\right)$ computes a $\mathbf{G b u}(G)$ derivation of $\tau$; we conclude $\vdash_{\mathrm{Gbu}(G)} G$.

As a corollary, we get the completeness of $\mathbf{F R J}(G)$ and $\mathbf{G b u}(G)$ :

\section{Theorem 10 (Completeness of FRJ $(G)$ and $\mathbf{G b u}(G)$ ).}

(i) $G \notin$ IPL implies $\vdash_{\mathbf{F R J}(G)} G$.

(ii) $G \in$ IPL implies $\vdash_{\mathbf{G b u}(G)} G$.

Proof. Let $G \notin \mathrm{IPL}$. By the Soundness of $\mathbf{G b u}(G)$ (Theorem 6 ), $\forall_{\mathbf{G b u}(G)} G$; by Theorem $9 . \vdash_{\mathbf{F R J}(G)} G$. Let $G \in \mathrm{IPL}$. By the Soundness of FRJ $(G)$ (Theorem 1), $\nvdash_{\mathbf{F R J}(G)} G$; by Theorem $9 . \vdash_{\mathbf{G b u}(G)} G$.

\section{Minimality}

In this section we prove that, given a non-valid formula $G$, one can build an FRJ $(G)$-derivation $\mathcal{D}$ of $G$ such that $\operatorname{Mod}(\mathcal{D})$ is a countermodel of $G$ having minimal height. Such a derivation can be constructed by tweaking the proofsearch procedure defined in Sec. 4 .

Let $G \notin \mathrm{IPL}$; the height of $G$, denoted by $\mathrm{h}(G)$, is the minimum height of a countermodel for $G$; formally:

$$
\mathrm{h}(G)=\min \{\mathrm{h}(\mathcal{K}) \mid \mathcal{K}=\langle P, \leq, \rho, V\rangle \text { and } \rho \nVdash G\}
$$

Note that $\mathrm{h}(G)=0$ iff $G$ is not classically valid. For instance, the formulas $S$ and $T$ of Ex. 5 have height 2, the formula $K$ of Ex. 6 has height 1 . Let $\mathcal{D}$ be an $\mathbf{F R J}(G)$-derivation of $G$. Since $\operatorname{Mod}(\mathcal{D})$ is a countermodel for $G$ (see Theorem 2), we have $\mathrm{h}(\operatorname{Mod}(\mathcal{D})) \geq \mathrm{h}(G)$. We show that we can build an $\mathbf{F R J}(G)$-derivation $\tilde{\mathcal{D}}$ of $G$ such that $\mathrm{h}(\operatorname{Mod}(\tilde{\mathcal{D}}))=\mathrm{h}(G)$. To this aim, we prove that:

(K1) given a countermodel $\mathcal{K}$ for $G$, there exists an $\mathbf{F R J}(G)$-derivation $\tilde{\mathcal{D}}$ of $G$ such that $\mathrm{h}(\operatorname{Mod}(\tilde{\mathcal{D}})) \leq \mathrm{h}(\mathcal{K})$.

By (K1) choosing as $\mathcal{K}$ a countermodel for $G$ having the minimal height $\mathrm{h}(G)$, we get an $\mathbf{F R J}(G)$-derivation $\tilde{\mathcal{D}}$ of $G$ such that $\mathrm{h}(\operatorname{Mod}(\tilde{\mathcal{D}}))=\mathrm{h}(G)$.

Let $\mathcal{D}$ be an $\mathbf{F R J}(G)$-derivation of $G$. The height of the countermodel $\operatorname{Mod}(\mathcal{D})$ is determined by the maximum number of applications of join rules along a branch of $\mathcal{D}$. To account for this, we introduce the notion of rank. Let $\sigma$ be a sequent occurring in $\mathcal{D}$; the rank of $\sigma$, denoted by $\operatorname{Rn}(\sigma)$, is inductively defined as follows:

- If $\sigma$ is an irregular axiom, then $\operatorname{Rn}(\sigma)=-1$.

- If $\sigma$ is a regular axiom, then $\operatorname{Rn}(\sigma)=0$. 
- If $\sigma$ is not an axiom, let

$$
\frac{\sigma_{1} \cdots \sigma_{n}}{\sigma} \mathcal{R}
$$

be the rule applied to get $\sigma(n \geq 1)$. Then:

$$
\operatorname{Rn}(\sigma)=\max \left\{\operatorname{Rn}\left(\sigma_{1}\right), \ldots, \operatorname{Rn}\left(\sigma_{n}\right)\right\}+c \quad \text { where } c= \begin{cases}1 & \text { if } \mathcal{R} \in\left\{\bowtie^{\mathrm{At}}, \bowtie^{\vee}\right\} \\ 0 & \text { otherwise }\end{cases}
$$

The rank of $\mathcal{D}$, denoted by $\operatorname{Rn}(\mathcal{D})$, is the rank of the root sequent of $\mathcal{D}$. One can easily prove that, for every regular sequent $\sigma$ in $\mathcal{D}, \operatorname{Rn}(\sigma)$ coincides with the height of the world $\phi(\sigma)$ in $\operatorname{Mod}(\sigma)$ (where $\phi$ is the map associated with $\mathcal{D})$. As an immediate consequence we get:

Lemma 13. Let $\mathcal{D}$ be an $\mathbf{F R J}(G)$-derivation of $G$. Then, $\operatorname{Rn}(\mathcal{D})=\mathrm{h}(\operatorname{Mod}(\mathcal{D}))$.

Let $\mathcal{K}=\langle P, \leq, \rho, V\rangle$ be a countermodel for $G$ and $\alpha \in P$. We set:

$-\alpha \Vdash^{*} H$ iff $\alpha \Vdash H$ and either $H \in \mathcal{V}$ or $H=A \supset B$ and $\alpha \nVdash A$.

$-\Lambda_{\alpha}=\{A \in \operatorname{SL}(G) \mid \alpha \Vdash A\}$.

$-\Lambda_{\alpha}^{*}=\left\{A \in \operatorname{SL}(G) \mid \alpha \Vdash^{*} A\right\}$.

$-\Omega_{\alpha}=\{C \in \operatorname{SR}(G) \mid \alpha \nVdash C\}$.

To prove (K1) we exploit the following lemma (the proof is deferred to the end of this section):

Lemma 14. Let $\mathcal{K}=\langle P, \leq, \rho, V\rangle$ be a countermodel for $G$, let $\alpha \in P$ and $C \in \Omega_{\alpha}$.

- There exists an $\mathbf{F R J}(G)$-derivation $\mathcal{D}_{\alpha}^{\rightarrow}(C)$ of $\sigma_{\alpha}^{\rightarrow}(C)=\Sigma ; \Theta \rightarrow C$ such that:

(i) $\operatorname{Rn}\left(\mathcal{D}_{\alpha}^{\rightarrow}(C)\right)<\mathrm{h}(\alpha)$;

(ii) $\Sigma \subseteq \Lambda_{\alpha}^{*} \subseteq \Sigma \cup \Theta$.

- There exists an $\mathbf{F R J}(G)$-derivation $\mathcal{D}_{\alpha}^{\Rightarrow}(C)$ of $\sigma_{\alpha}^{\Rightarrow}(C)=\Gamma \Rightarrow C$ such that:

(iii) $\operatorname{Rn}\left(\mathcal{D}_{\alpha}^{\Rightarrow}(C)\right) \leq \mathrm{h}(\alpha)$;

(iv) there is $\beta \in P$ such that $\alpha \leq \beta$ and $\Lambda_{\beta}^{*} \subseteq \Gamma$.

Point (K1) follows by the above lemma, taking as $\tilde{\mathcal{D}}$ the $\mathbf{F R J}(G)$-derivation $\mathcal{D} \Rightarrow(G)$ associated with the root $\rho$ of $\mathcal{K}$. Indeed, the root sequent of $\tilde{\mathcal{D}}$ is $\sigma_{\rho}(G)=$ $\Gamma \Rightarrow G$, hence $\tilde{\mathcal{D}}$ is an $\mathbf{F R J}(G)$-derivation of $G$. By Theorem 2 $\operatorname{Mod}(\tilde{\mathcal{D}})$ is a countermodel for $G$ and, by point (iii) of the lemma, $\operatorname{Rn}(\tilde{\mathcal{D}}) \leq \mathrm{h}(\rho)$, namely $\operatorname{Rn}(\tilde{\mathcal{D}}) \leq \mathrm{h}(\mathcal{K})$. By Lemma 13 , we get $\mathrm{h}(\operatorname{Mod}(\tilde{\mathcal{D}})) \leq \mathrm{h}(\mathcal{K})$, which proves $(\mathrm{K} 1)$, As discussed above, from (K1) we conclude:

Theorem 11. Let $G \notin \mathrm{IPL}$. Then, there exists an $\mathbf{F R J}(G)$-derivation $\tilde{\mathcal{D}}$ of $G$ such that $\mathrm{h}(\operatorname{Mod}(\tilde{\mathcal{D}}))=\mathrm{h}(G)$. 
We remark that Theorem 11 provides an alternative proof of the completeness of $\mathbf{F R J}(G) \cdot{ }^{4}$

We have now to prove Lemma 14. We give a constructive proof to define the sequents $\sigma_{\alpha}(C)$ and $\sigma_{\alpha}^{\Rightarrow}(C)$ and the related derivations, which relies on the following strategy:

- we visit the model $\mathcal{K}$ top-down, considering the worlds $\alpha$ of $\mathcal{K}$ in increasing order of height;

- for each world $\alpha$, we firstly define all the irregular sequents $\sigma_{\alpha} \rightarrow(C)$ and then all the regular sequents $\sigma_{\alpha}^{\Rightarrow}(C)$;

- we pick the formulas $C$ of $\Omega_{\alpha}$ in increasing order of size.

Since $\rho$ is the bottom world of $\mathcal{K}$ and $G$ is the formula of maximum size in $\Omega_{G}$, the FRJ $(G)$-derivation $\mathcal{D}_{\rho}^{\Rightarrow}(G)$ is obtained at the end of the process. To highlight the main insights of the above construction, we present some examples.

Example 9. Let $S$ be the instance of Scott Principle in Ex. 5 .

$$
\begin{aligned}
& S=H \supset \neg \neg p \vee \neg p \quad H=(\neg \neg p \supset p) \supset \neg p \vee p \\
& \mathrm{SL}(S)=\{H, \neg p \vee p, \neg \neg p, \neg p, p\} \\
& \mathrm{SR}(S)=\{S, \neg \neg p \vee \neg p, \neg \neg p \supset p, \neg \neg p, \neg p, p, \perp\}
\end{aligned}
$$

We have $\mathrm{h}(S)=2$. Let us consider the countermodel $\mathcal{K}_{S}$ for $S$ having height 2 depicted below, consisting of the worlds $\alpha$ and $\beta$ of height 0 , the world $\gamma$ of height 1 and the root $\rho$ of height 2 :

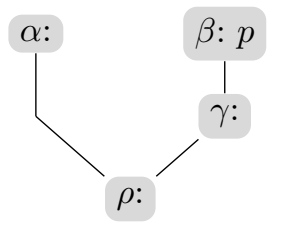

We define the sequents $\sigma_{\alpha}(C)$ and $\sigma_{\alpha}^{\Rightarrow}(C)$ matching Lemma 14. For each sequent $\sigma$, we display the rule applied to obtain $\sigma$ and the rank of $\sigma$. Using these annotations, one can immediately build the derivations $\mathcal{D}_{\alpha}^{\rightarrow}(C)$ and $\mathcal{D}_{\alpha}^{\Rightarrow}(C)$. We traverse the model $\mathcal{K}$ downwards, starting from the world $\alpha$ of height 0 . We have:

$$
\Lambda_{\alpha}^{*}=\{\neg p\} \quad \Lambda_{\alpha}=\Lambda_{\alpha}^{*} \cup\{\neg p \vee p, H\} \quad \Omega_{\alpha}=\{\perp, p, \neg \neg p\}
$$

Note that $H \notin \Lambda_{\alpha}^{*}$ since $\alpha \Vdash \neg \neg p \supset p$. Firstly, we define all the sequents $\sigma_{\alpha}(C)$, where $C \in \Omega_{\alpha}$; formulas $C$ are considered in increasing order of size.

\begin{tabular}{lc|l|l} 
Sequent & & Applied rule & Rank \\
\hline$\sigma_{\alpha}(\perp)$ & $; p, H, \neg \neg p, \neg p \rightarrow \perp$ & $\mathrm{Ax}_{\rightarrow}$ & -1 \\
$\sigma_{\alpha}(p)$ & $\cdot ; H, \neg \neg p, \neg p \rightarrow p$ & $\mathrm{Ax}_{\rightarrow}$ & -1 \\
$\sigma_{\alpha}^{\rightarrow}(\neg \neg p)$ & $\neg p ; p, H, \neg \neg p \rightarrow \neg \neg p$ & $\supset \in \sigma_{\alpha}(\perp)$ & -1
\end{tabular}

\footnotetext{
$\overline{4}$ Actually, in [12] the completeness of $\mathbf{F R J}(G)$ has been proved along these lines.
} 
Secondly, we define the sequents $\sigma_{\alpha}^{\Rightarrow}(C)$, where $C \in \Omega_{\alpha}$.

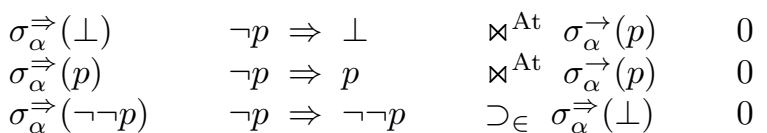

Let us consider the world $\beta$ of height 0 . We have:

$$
\Lambda_{\beta}^{*}=\{p, \neg \neg p\} \quad \Lambda_{\beta}=\Lambda_{\beta}^{*} \cup\{\neg p \vee p, H\} \quad \Omega_{\beta}=\{\perp, \neg p\}
$$

For $C \in \Omega_{\beta}$, we define the sequents $\sigma_{\beta}(C)$ :

$$
\begin{array}{lrll}
\sigma_{\beta}(\perp) & ; p, H, \neg \neg p, \neg p \rightarrow \perp & \mathrm{Ax}_{\rightarrow} & -1 \\
\sigma_{\beta}(\neg p) & p ; H, \neg \neg p, \neg p \rightarrow \neg p & \supset \in \sigma_{\beta}(\perp)-1
\end{array}
$$

For $C \in \Omega_{\beta}$, sequents $\sigma_{\beta}(C)$ are:

$$
\begin{array}{llll}
\sigma_{\beta}(\perp) & p, \neg \neg p \Rightarrow \perp & \bowtie^{\text {At }} \sigma_{\beta}(\neg p) & 0 \\
\sigma_{\beta}^{\Rightarrow}(\neg p) & p, \neg \neg p \Rightarrow \neg p & \supset \in \sigma_{\beta}(\perp) & 0
\end{array}
$$

Let us consider $\gamma$, the only world of height 1 . We have:

$$
\Lambda_{\gamma}^{*}=\Lambda_{\gamma}=\{H, \neg \neg p\} \quad \Omega_{\gamma}=\{\perp, p, \neg p, \neg \neg p \supset p\}
$$

For $C \in \Omega_{\gamma}$, sequents $\sigma_{\gamma}(C)$ are:

$$
\begin{array}{lrll}
\sigma_{\gamma}(\perp) & ; p, H, \neg \neg p, \neg p \rightarrow \perp & & -1 \\
\sigma_{\gamma}^{\rightarrow}(p) & \cdot H, \neg \neg p, \neg p \rightarrow p & \mathrm{Ax}_{\rightarrow} & -1 \\
\sigma_{\gamma}^{\rightarrow}(\neg p) & \cdot ; H, \neg \neg p \rightarrow \neg p & \mathrm{Ax}_{\rightarrow} & 0 \\
\sigma_{\gamma}^{\rightarrow}(\neg \neg p \supset p) & \neg \neg p ; H, \neg p \rightarrow \neg \sigma_{\beta}(\perp) & 0
\end{array}
$$

For $C \in \Omega_{\gamma}$, sequents $\sigma_{\gamma}^{\Rightarrow}(C)$ are:

$$
\begin{aligned}
\sigma_{\gamma}^{\Rightarrow}(\perp) & p, \neg \neg p & \Rightarrow \perp & & \bowtie^{\text {At }} \sigma_{\beta}(\neg p) & 0 \\
\sigma_{\gamma}^{\Rightarrow}(p) & H, \neg \neg p & \Rightarrow p & & \bowtie^{\text {At }} \sigma_{\gamma}(\neg p) \sigma_{\gamma}(\neg \neg p \supset p) & 1 \\
\sigma_{\gamma}^{\Rightarrow}(\neg p) & p, \neg \neg p & \Rightarrow \neg p & & \supset_{\in} \sigma_{\beta}^{\Rightarrow}(\perp) & 0 \\
\sigma_{\gamma}^{\Rightarrow}(\neg \neg p \supset p) & H, \neg \neg p & \Rightarrow \neg \neg p \supset p & & \supset_{\in} \sigma_{\gamma}^{\Rightarrow}(p) &
\end{aligned}
$$

Finally, let us consider the root $\rho$ having height 2. We have:

$$
\Lambda_{\rho}^{*}=\Lambda_{\rho}=\{H\} \quad \Omega_{\rho}=\operatorname{SR}(S)
$$

We can inherit the following definitions from the worlds $\alpha, \beta, \gamma$ :

$$
\begin{array}{lcc}
\sigma_{\rho}(\perp)=\sigma_{\alpha}(\perp) & \sigma_{\rho}(p)=\sigma_{\gamma}(p) & \sigma_{\rho}^{\rightarrow}(\neg p)=\sigma_{\gamma}(\neg p) \\
\sigma_{\rho}^{\Rightarrow}(\perp)=\sigma_{\beta}(\perp) & \sigma_{\rho}^{\Rightarrow}(p)=\sigma_{\alpha}^{\Rightarrow}(p) & \sigma_{\rho}^{\Rightarrow}(\neg p)=\sigma_{\beta}(\neg p) \\
\sigma_{\rho}^{\Rightarrow}(\neg \neg p)=\sigma_{\alpha}(\neg \neg p) & \sigma_{\rho}^{\Rightarrow}(\neg \neg p \supset p)=\sigma_{\gamma}^{\Rightarrow}(\neg \neg p \supset p)
\end{array}
$$


For $C \in\{\neg \neg p, \neg \neg p \supset p, \neg \neg p \vee \neg p, S\}$, the sequents $\sigma_{\rho}(C)$ are:

$$
\begin{array}{llll}
\sigma_{\rho}(\neg \neg p) & ; H \rightarrow \neg \neg p & \supset_{\notin} \sigma_{\alpha}(\perp) & 0 \\
\sigma_{\rho}(\neg \neg p \supset p) & \cdot ; H \rightarrow \neg \neg p \supset p & \supset_{\nexists} \sigma_{\gamma}(p) & 1 \\
\sigma_{\rho}(\neg \neg p \vee \neg p) & ; H \rightarrow \neg \neg p \vee \neg p & \vee \sigma_{\rho}(\neg \neg p) \sigma_{\gamma}(\neg p) & 0 \\
\sigma_{\rho}(S) & H ; \cdot \rightarrow S & \supset \in \sigma_{\rho}(\neg \neg p \vee \neg p) & 0
\end{array}
$$

For $C \in\{\neg \neg p \vee \neg p, S\}$, the sequents $\sigma_{\bar{\rho}}(C)$ are:

$$
\begin{aligned}
& \sigma_{\rho}^{\Rightarrow}(\neg \neg p \vee \neg p) \quad H \Rightarrow \neg \neg p \vee \neg p \quad \bowtie^{\vee} \sigma_{\rho}(\neg \neg p \supset p) \sigma_{\rho}(\neg \neg p) \sigma_{\gamma}(\neg p) \quad 2 \\
& \underset{\sigma_{\rho}}{\Rightarrow}(S) \quad H \Rightarrow S \quad \supset_{\in} \sigma_{\rho}^{\Rightarrow}(\neg \neg p \vee \neg p) \quad 2
\end{aligned}
$$

The FRJ $(S)$-derivation $\mathcal{D}_{\rho}^{\Rightarrow}(S)$ of $\sigma_{\rho}^{\Rightarrow}(S)$ obtained in the end is an $\mathbf{F R J}(S)$ derivation of the goal $S$. Note that $\mathcal{D}_{\rho}^{\Rightarrow}(S)$ essentially coincides with the derivation in Fig. 2, hence the model $\operatorname{Mod}\left(\mathcal{D}_{\rho} \Rightarrow(S)\right)$ extracted from $\mathcal{D}_{\rho}^{\Rightarrow}(S)$ is isomorphic to the countermodel $\mathcal{K}_{S}$ displayed at the beginning of this example.

In the previous example, the model $\mathcal{K}_{S}$ initially chosen is a minimal countermodel for $S$, since there exists no countermodel for $S$ having less than 4 worlds, and the obtained model $\operatorname{Mod}\left(\mathcal{D}_{\rho}^{\Rightarrow}(S)\right)$ is a minimal countermodel for $S$ as well. One may wonder if this is always the case. The answer is negative, as shown in the next example; we also point out that Lemma 14 only sets a bound on the height of $\operatorname{Mod}\left(\mathcal{D}_{\rho}^{\Rightarrow}(G)\right)$ and not on the number of worlds.

Example 10. Let $C$ be the formula

$$
C=A \vee B \quad A=\left(p_{1} \supset p_{2}\right) \vee\left(p_{2} \supset p_{1}\right) \quad B=\left(q_{1} \supset q_{2}\right) \vee\left(q_{2} \supset q_{1}\right)
$$

We have $\mathrm{h}(C)=1$. Let $\mathcal{K}_{C}$ be the following countermodel for $C$ of height 1 , consisting of the worlds $\alpha$ and $\beta$ of height 0 and of the root $\rho$ of height 1 :

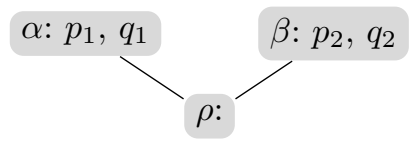

One can easily check that there is no countermodel for $C$ having less than 3 worlds, hence $\mathcal{K}_{C}$ is a minimal countermodel for $C$. We have:

$$
\begin{aligned}
& \operatorname{SL}(C)=\left\{p_{1}, p_{2}, q_{1}, q_{2}\right\} \\
& \operatorname{SR}(C)=\left\{C, A, B, p_{1} \supset p_{2}, p_{2} \supset p_{1}, q_{1} \supset q_{2}, q_{2} \supset q_{1}, p_{1}, p_{2}, q_{1}, q_{2}\right\} \\
& \Lambda_{\alpha}^{*}=\Lambda_{\alpha}=\left\{p_{1}, q_{1}\right\} \quad \Omega_{\alpha}=\left\{p_{2}, q_{2}, p_{1} \supset p_{2}, q_{1} \supset q_{2}\right\} \\
& \Lambda_{\beta}^{*}=\Lambda_{\beta}=\left\{p_{2}, q_{2}\right\} \quad \Omega_{\beta}=\left\{p_{1}, q_{1}, p_{2} \supset p_{1}, q_{2} \supset q_{1}\right\} \\
& \Lambda_{\rho}^{*}=\Lambda_{\rho}=\emptyset \quad \Omega_{\rho}=\operatorname{SR}(C)
\end{aligned}
$$

We define the sequents $\sigma_{\delta}(C)$ and $\sigma_{\delta}(C)$, where $\delta \in\{\alpha, \beta, \rho\}$ and $C \in \Omega_{\delta}$, only showing the sequents needed to prove the goal. 


\begin{tabular}{|c|c|c|c|}
\hline $\begin{array}{l}\sigma_{\alpha}^{\Rightarrow}\left(p_{2}\right) \\
\sigma_{\alpha}^{\Rightarrow}\left(q_{2}\right)\end{array}$ & $\begin{array}{l}p_{1}, q_{1}, q_{2} \\
p_{1}, p_{2}, q_{1}\end{array}$ & $\begin{array}{l}\Rightarrow p_{2} \\
\Rightarrow q_{2}\end{array}$ & $\begin{array}{l}\mathrm{Ax}_{\Rightarrow} \\
\mathrm{Ax}_{\Rightarrow} \Rightarrow\end{array}$ \\
\hline $\overrightarrow{\sigma_{\beta}}\left(p_{1}\right)$ & $p_{2}, q_{1}, q_{2}$ & $\Rightarrow p_{1}$ & $\mathrm{Ax}_{\Rightarrow}$ \\
\hline$\sigma_{\beta} \vec{\beta}\left(q_{1}\right)$ & $p_{1}, p_{2}, q_{2}$ & $\Rightarrow q_{1}$ & $\mathrm{Ax}_{\Rightarrow}$ \\
\hline $\overrightarrow{\sigma_{\rho}}\left(p_{1} \supset p_{2}\right)$ & $\cdot ; q_{1}, q_{2}$ & $\rightarrow p_{1} \supset p_{2}$ & $\supset_{\notin} \sigma_{\alpha}^{\Rightarrow}\left(p_{2}\right)$ \\
\hline$\sigma_{\rho}^{\rightarrow}\left(p_{2} \supset p_{1}\right)$ & $\cdot ; q_{1}, q_{2}$ & $\rightarrow p_{2} \supset p_{1}$ & $\supset_{\notin} \sigma_{\beta}\left(p_{1}\right)$ \\
\hline$\sigma_{\rho}\left(q_{1} \supset q_{2}\right)$ & $\cdot ; p_{1}, p_{2}$ & $\rightarrow q_{1} \supset q_{2}$ & $\supset_{\notin} \sigma_{\alpha}^{\Rightarrow}\left(q_{2}\right)$ \\
\hline$\sigma_{\rho}^{\rightarrow}\left(q_{2} \supset q_{1}\right)$ & $\cdot ; p_{1}, p_{2}$ & $\rightarrow q_{2} \supset q_{1}$ & $\supset_{\notin} \sigma_{\vec{\beta}}\left(q_{1}\right)$ \\
\hline$\sigma_{\rho}^{\rightarrow}(A)$ & $\cdot ; q_{1}, q_{2}$ & $\rightarrow A$ & $\vee \sigma_{\rho}\left(p_{1} \supset p_{2}\right) \sigma_{\rho}\left(p_{2} \supset p_{1}\right)$ \\
\hline$\sigma_{\rho}^{\rightarrow}(B)$ & $\cdot ; p_{1}, p_{2}$ & $\rightarrow B$ & $\vee \sigma_{\rho}^{\rightarrow}\left(q_{1} \supset q_{2}\right) \sigma_{\rho}^{\rightarrow}\left(q_{2} \supset q_{1}\right)$ \\
\hline $\overrightarrow{\sigma_{\rho}}(C)$ & & $\Rightarrow A \vee B$ & $\star^{\vee} \sigma_{\rho}(A) \sigma_{\rho}(B)$ \\
\hline
\end{tabular}

The goal $C$ is proved by the $\mathbf{F R J}(C)$-derivation $\mathcal{D}_{\rho}^{\Rightarrow}(C)$ of $\sigma_{\rho}^{\Rightarrow}(C)$. The model $\operatorname{Mod}\left(\mathcal{D}_{\rho}^{\Rightarrow}(C)\right)$ extracted from $\mathcal{D}_{\rho}^{\Rightarrow}(C)$ is:

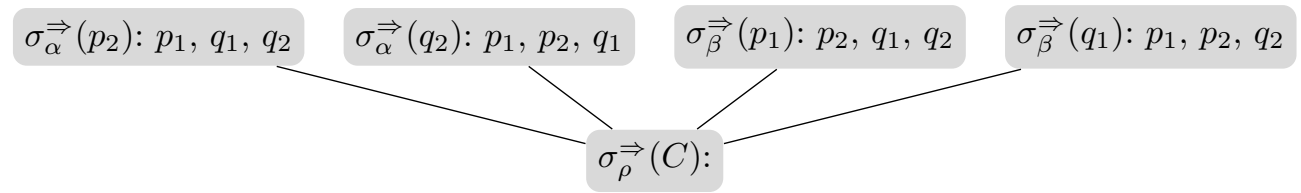

Such a model has the minimal height 1 , as expected, but it is not minimal. To obtain minimal models, we should redesign FRJ $(G)$ in a multi-succedent style, so that axioms of the kind $p_{1}, q_{1} \Rightarrow p_{2}, q_{2}$ and $p_{2}, q_{2} \Rightarrow p_{1}, q_{1}$ are allowed. Thus, we should have regular axioms of the kind $\Gamma^{\mathrm{At}} \Rightarrow \Delta^{\mathrm{At}}$, where $\Gamma^{\mathrm{At}}$ and $\Delta^{\text {At }}$ are disjoint sets of propositional variables such that $\Gamma^{\text {At }} \subseteq \operatorname{Lhs}(G)$ and $\Delta^{\text {At }} \subseteq \operatorname{Rhs}(G)$. However, this would cause an exponential blow-up of the number of provable sequents and proof-search would become unfeasible. E.g., in this case we would get 64 regular axioms instead of 5 .

We remark that we can modify the proof-search procedure of Fig. 8 so to generate $\mathbf{F R J}(G)$-derivations of minimal height; this is obtained by delaying the application of join rules as much as possible, mimicking the strategy applied in the above examples.

The rest of this section is devoted to the detailed proof of Lemma 14. Firstly, we prove some closure properties of sets $\Lambda_{\alpha}$ and $\Lambda_{\alpha}^{*}$ :

Lemma 15. Let $\mathcal{K}$ be a countermodel for $G$ and $\alpha$ a world of $\mathcal{K}$. Then, $\Lambda_{\alpha}=$ $\mathcal{C l}\left(\Lambda_{\alpha}\right)=\mathcal{C l}\left(\Lambda_{\alpha}^{*}\right)$.

Proof. To prove the assertion, we show that:

(i) $\Lambda_{\alpha} \subseteq \mathcal{C l}\left(\Lambda_{\alpha}\right)$.

(ii) $\mathcal{C l}\left(\Lambda_{\alpha}\right) \subseteq \Lambda_{\alpha}$.

(iii) $\mathcal{C l}\left(\Lambda_{\alpha}^{*}\right) \subseteq \mathcal{C l}\left(\Lambda_{\alpha}\right)$. 
(iv) $\Lambda_{\alpha} \subseteq \mathcal{C l}\left(\Lambda_{\alpha}^{*}\right)$.

(v) $\mathcal{C l}\left(\Lambda_{\alpha}\right) \subseteq \mathcal{C l}\left(\Lambda_{\alpha}^{*}\right)$.

Point (i) immediately follows by (Cl3) To prove(ii), let $C \in \mathcal{C l}\left(\Lambda_{\alpha}\right)$; by induction on $|C|$, we show that $C \in \Lambda_{\alpha}$. The case $C \in \mathcal{V}^{\perp}$ immediately follows by $(\mathcal{C l} l 5)$. Let $C=A \wedge B$. Then, $A \wedge B \in \Lambda_{\alpha}$ or $\{A, B\} \subseteq \mathcal{C l}\left(\Lambda_{\alpha}\right)$. In the former case, we are done. In the latter case, by the induction hypothesis we have $\{A, B\} \subseteq \Lambda_{\alpha}$. Thus $\alpha \Vdash A$ and $\alpha \Vdash B$, which implies $\alpha \Vdash A \wedge B$, hence $A \wedge B \in \Lambda_{\alpha}$. The cases $C=A \vee B$ and $C=A \supset B$ are similar.

Point (iii) follows by the fact that $\Lambda_{\alpha}^{*} \subseteq \Lambda_{\alpha}$ and by $(\mathcal{C l}$ ).

To prove [iv)] let $C \in \Lambda_{\alpha}$, namely $\alpha \Vdash C$; by induction on $|C|$, we show that $C \in \mathcal{C l}\left(\Lambda_{\alpha}^{*}\right)$. If $C \in \mathcal{V}$, then $\alpha \Vdash^{*} C$, hence $C \in \Lambda_{\alpha}^{*}$, which implies $C \in \mathcal{C l}\left(\Lambda_{\alpha}^{*}\right)$. Let $C=A \supset B$. If $\alpha \nVdash A$, then $\alpha \Vdash^{*} C$ and, as above, $C \in \mathcal{C l}\left(\Lambda_{\alpha}^{*}\right)$. If $\alpha \Vdash A$, then $\alpha \Vdash B$; by induction hypothesis, $B \in \mathcal{C l}\left(\Lambda_{\alpha}^{*}\right)$, hence $A \supset B \in \mathcal{C l}\left(\Lambda_{\alpha}^{*}\right)$. The cases $C=A \wedge B$ and $C=A \vee B$ easily follow by the induction hypothesis. Point (v) immediately follows by (iv) and by (Cl6).

To sum up, by (i) and (ii) we get $\Lambda_{\alpha}=\mathcal{C} l\left(\Lambda_{\alpha}\right)$; by (iii) and (v), $\mathcal{C l}\left(\Lambda_{\alpha}\right)=$ $\mathcal{C l}\left(\Lambda_{\alpha}^{*}\right)$.

Now, let us come to the proof of Lemma 14

Proof ((Lemma 14)). We define the derivation $\mathcal{D}_{\alpha}^{\odot}(C)$, where $\odot \in\{\Rightarrow, \rightarrow\}$, using the following mutual induction hypothesis (mirroring the order used to define the sequents $\sigma_{\alpha}^{\rightarrow}(C)$ and $\sigma_{\alpha}^{\Rightarrow}(C)$ in Ex. 9 and $10 p$ :

(IH1) a main induction on $\mathrm{h}(\alpha)$;

(IH2) a secondary induction on $\operatorname{tp}^{-}(\odot)$, where $\operatorname{tp}^{-}(\rightarrow)=0$ and $\operatorname{tp}^{-}(\Rightarrow)=1$;

(IH3) a third induction on $|C|$.

Let us introduce the following notations:

$$
\begin{array}{ll}
\bar{\Gamma}^{\mathrm{At}}=\operatorname{SL}(G) \cap \mathcal{V} & \bar{\Gamma}^{\supset}=\operatorname{SL}(G) \cap \mathcal{L}^{\supset} \quad \bar{\Gamma}=\bar{\Gamma}^{\mathrm{At}} \cup \bar{\Gamma}^{\supset} \\
\Lambda_{\alpha}^{* \mathrm{At}}=\Lambda_{\alpha}^{*} \cap \mathcal{V} & \Lambda_{\alpha}^{* \supset}=\Lambda_{\alpha}^{*} \cap \mathcal{L}^{\supset}
\end{array}
$$

We proceed by a case analysis on $C$ and $\mathcal{D}_{\alpha}^{\odot}(C)$. We point out that, since $\alpha \nVdash C$, then $C \notin \Lambda_{\alpha}$ and $C \notin \Lambda_{\alpha}^{*}$.

- Case $C \in \mathcal{V}^{\perp}$, definition of $\mathcal{D}_{\alpha}^{\rightarrow}(C)$.

We set:

$$
\mathcal{D}_{\alpha}^{\rightarrow}(C)=\overline{\sigma_{\alpha}(C)=\cdot ; \bar{\Gamma}^{\mathrm{At}} \backslash\{C\}, \bar{\Gamma}^{\supset} \rightarrow C} \mathrm{Ax}_{\rightarrow}
$$

Since $\operatorname{Rn}\left(\mathcal{D}_{\alpha}^{\rightarrow}(C)\right)=-1$ and $\mathrm{h}(\alpha) \geq 0$, we have $\operatorname{Rn}\left(\mathcal{D}_{\alpha}^{\rightarrow}(C)\right)<\mathrm{h}(\alpha)$, hence (i) holds. Since $C \notin \Lambda_{\alpha}^{*}$, Point (ii) immediately follows.

- Case $C \in \mathcal{V}^{\perp}$, definition of $\mathcal{D}_{\alpha}^{\Rightarrow}(C)$. 
If $\Lambda_{\alpha}^{* \supset}$ is empty, namely $\Lambda_{\alpha}^{*}=\Lambda_{\alpha}^{* \text { At }}$, we set:

$$
\mathcal{D}_{\alpha}^{\Rightarrow}(C)=\overline{\sigma_{\alpha}^{\Rightarrow}(C)=\bar{\Gamma}^{\mathrm{At}} \backslash\{C\} \Rightarrow C} \mathrm{Ax}_{\Rightarrow}
$$

Since $\operatorname{Rn}\left(\mathcal{D}_{\alpha}^{\Rightarrow}(C)\right)=0$, we have $\operatorname{Rn}\left(\mathcal{D}_{\alpha}^{\Rightarrow}(C)\right) \leq \mathrm{h}(\alpha)$, which proves (iii). Point (iv) holds for $\beta=\alpha$ since $C \notin \Lambda_{\alpha}^{*}$. If $\Lambda_{\alpha}^{* \supset}$ is nonempty let

$$
\Upsilon=\left\{Y \mid Y \supset Z \in \Lambda_{\alpha}^{* \supset}\right\}=\left\{A_{1}, \ldots, A_{n}\right\} \quad(n \geq 1) .
$$

By definition of $\Lambda_{\alpha}^{* \supset}$, we have $\alpha \nVdash A_{j}$, for every $A_{j} \in \Upsilon$. By (IH2), for every $1 \leq j \leq n$, there is an $\mathbf{F R J}(G)$-derivation $\mathcal{D}_{\alpha}\left(A_{j}\right)$ of $\sigma_{\alpha}\left(A_{j}\right)=\Sigma_{j} ; \Theta_{j} \rightarrow A_{j}$ such that:

(P1) $\operatorname{Rn}\left(\mathcal{D}_{\alpha}^{\rightarrow}\left(A_{j}\right)\right)<\mathrm{h}(\alpha)$;

(P2) $\Sigma_{j} \subseteq \Lambda_{\alpha}^{*} \subseteq \Sigma_{j} \cup \Theta_{j}$.

We stress that the use of (IH2) is sound since $\operatorname{tp}^{-}(\rightarrow)<\operatorname{tp}^{-}(\Rightarrow)$. Let $\Sigma_{j}=$ $\Sigma_{j}^{\text {At }} \cup \Sigma_{j}^{\supset}$ and $\Theta_{j}=\Theta_{j}^{\text {At }} \cup \Theta_{j}^{\supset}$. We prove that $\sigma_{\alpha}\left(A_{1}\right), \ldots, \sigma_{\alpha}\left(A_{n}\right)$ satisfy the following properties, for every $1 \leq j \leq n$ :

(a) $\Sigma_{i} \subseteq \Sigma_{j} \cup \Theta_{j}$, for every $i \neq j$;

(b) $Y \supset Z \in \Sigma_{j}^{\supset}$ implies $Y \in \Upsilon$;

(c) $C \notin \Sigma_{j}^{\mathrm{At}}$.

Point (a) follows by (P2) Point (b) follows by (P2) and the definition of $\Upsilon$. Point $\left[\right.$ (c) follows by the fact that $C \notin \Lambda_{\alpha}^{*}$ and (P2) By (a) (c) we can apply the rule $\bowtie^{\mathrm{At}}$ with premises $\sigma_{\alpha}\left(A_{1}\right), \ldots, \sigma_{\alpha}\left(A_{n}\right)$ and build the $\mathbf{F R J}(G)$-derivation $\mathcal{D}_{\alpha}^{\Rightarrow}(C)$ displayed below $\left(\Sigma^{\mathrm{At}}, \Sigma^{\supset}, \Theta^{\mathrm{At}}, \Theta^{\supset}\right.$ are defined as in Fig. 1):

$$
\begin{gathered}
\mathcal{D}_{\alpha}^{\rightarrow}\left(A_{j}\right) \\
\frac{\ldots \Sigma_{j}^{\mathrm{At}}, \Sigma_{j}^{\supset} ; \Theta_{j}^{\mathrm{At}}, \Theta_{j}^{\supset} \rightarrow A_{j} \ldots}{\sigma_{\alpha}^{\Rightarrow}(C)=\Gamma \Rightarrow C} \bowtie^{\mathrm{At}} \quad j=1 \ldots n \\
\Gamma=\Sigma^{\mathrm{At}} \cup\left(\Theta^{\mathrm{At}} \backslash\{C\}\right) \cup \Sigma^{\supset} \cup \Theta^{\supset}
\end{gathered}
$$

Note that, by the definition of $\Upsilon$, the application of $\bowtie^{\text {At }}$ satisfies the restriction (PS3) stated in Sec. 3. By definition, $\operatorname{Rn}\left(\mathcal{D}_{\alpha}^{\Rightarrow}(C)\right)=m+1$, where $m$ is the maximum among $\operatorname{Rn}\left(\mathcal{D}_{\alpha}^{\rightarrow}\left(A_{1}\right)\right), \ldots, \operatorname{Rn}\left(\mathcal{D}_{\alpha}^{\rightarrow}\left(A_{n}\right)\right)$. By (P1) $m<\mathrm{h}(\alpha)$, hence $\operatorname{Rn}\left(\mathcal{D}_{\alpha}^{\Rightarrow}(C)\right) \leq \mathrm{h}(\alpha)$, and this proves (iii). We show that $\Lambda_{\alpha}^{*} \subseteq \Gamma$, and this proves (iv) (where $\beta=\alpha$ ). If, for some $j \in\{1, \ldots, n\}, \Lambda_{\alpha}^{*} \subseteq \Sigma_{j}$, then $\Lambda_{\alpha}^{*} \subseteq \Sigma^{\text {At }} \cup \Sigma^{S}$, hence $\Lambda_{\alpha}^{*} \subseteq \Gamma$. Otherwise, by (P2) $\Lambda_{\alpha}^{*} \subseteq \bigcap_{1 \leq j \leq n} \Theta_{j}$, which implies:

(d) $\Lambda_{\alpha}^{* \mathrm{At}} \subseteq \bigcap_{1 \leq j \leq n} \Theta_{j}^{\mathrm{At}}$;

(e) $\Lambda_{\alpha}^{* \supset} \subseteq \bigcap_{1 \leq j \leq n} \Theta_{j}^{\supset}$. 
Since $C \notin \Lambda_{\alpha}^{* \mathrm{At}}$ and $\bigcap_{1 \leq j \leq n} \Theta_{j}^{\mathrm{At}}=\Theta^{\mathrm{At}}$, by (d) we get $\Lambda_{\alpha}^{* \mathrm{At}} \subseteq \Theta^{\mathrm{At}} \backslash\{C\}$, hence $\Lambda_{\alpha}^{* \text { At }} \subseteq \Gamma$. Moreover, since $Y \supset Z \in \Lambda_{\alpha}^{*}$ implies $Y \in \Upsilon$, by (e) we get $\Lambda_{\alpha}^{* \supset} \subseteq\left(\bigcap_{1 \leq j \leq n} \Theta_{j}^{\supset}\right) / \Upsilon$, namely $\Lambda_{\alpha}^{* \supset} \subseteq \Theta^{\supset}$, hence $\Lambda_{\alpha}^{* \supset} \subseteq \Gamma$. We conclude that $\Lambda_{\alpha}^{*}=\Lambda_{\alpha}^{* \text { At }} \cup \Lambda_{\alpha}^{* \supset} \subseteq \Gamma$.

- Case $C=C_{1} \vee C_{2}$, definition of $\mathcal{D}_{\alpha}^{\rightarrow}(C)$.

Since $\alpha \nVdash C_{1} \vee C_{1}$, we have $\alpha \nVdash C_{1}$ and $\alpha \nVdash C_{2}$. By (IH3)] for every $k \in\{1,2\}$ there is an $\mathbf{F R J}(G)$-derivation $\mathcal{D}_{\alpha}\left(C_{k}\right)$ of $\sigma_{\alpha}\left(C_{k}\right)=\Sigma_{k} ; \Theta_{k} \rightarrow C_{k}$ such that

(Q1) $\operatorname{Rn}\left(\mathcal{D}_{\alpha}^{\rightarrow}\left(C_{k}\right)\right)<\mathrm{h}(\alpha)$;

(Q2) $\Sigma_{k} \subseteq \Lambda_{\alpha}^{*} \subseteq \Sigma_{k} \cup \Theta_{k}$.

By (Q2) we get $\Sigma_{1} \subseteq \Sigma_{2} \cup \Theta_{2}$ and $\Sigma_{2} \subseteq \Sigma_{1} \cup \Theta_{1}$, hence we can set:

$$
\begin{aligned}
& \mathcal{D}_{\alpha}^{\rightarrow}\left(C_{1}\right) \quad \mathcal{D}_{\alpha} \rightarrow\left(C_{2}\right) \\
& \mathcal{D}_{\alpha}^{\rightarrow}(C)=\frac{\Sigma_{1} ; \Theta_{1} \rightarrow C_{1}}{\sigma_{\alpha}^{\rightarrow}(C)=\underbrace{\Sigma_{1}, \Sigma_{2}}_{\Sigma} ; \underbrace{\Theta_{1} \cap \Theta_{2}}_{\Theta} \rightarrow C_{1} \vee C_{2}} \vee
\end{aligned}
$$

Since $\operatorname{Rn}\left(\mathcal{D}_{\alpha}^{\rightarrow}(C)\right)$ is the maximum between $\operatorname{Rn}\left(\mathcal{D}_{\alpha}^{\rightarrow}\left(C_{1}\right)\right)$ and $\operatorname{Rn}\left(\mathcal{D}_{\alpha} \rightarrow\left(C_{2}\right)\right)$, by (Q1) we get $\operatorname{Rn}\left(\mathcal{D}_{\alpha}(C)\right)<\mathrm{h}(\alpha)$, and this proves (i) By (Q2), we immediately get $\Sigma \subseteq \Lambda_{\alpha}^{*}$. Moreover, by (Q2)] it follows that $\Lambda_{\alpha}^{*} \subseteq \Sigma_{1}$ or $\Lambda_{\alpha}^{*} \subseteq \Sigma_{2}$ or $\Lambda_{\alpha}^{*} \subseteq \Theta_{1} \cap \Theta_{2}$, which implies $\Lambda_{\alpha}^{*} \subseteq \Sigma \cup \Theta$. Thus, (ii) holds.

- Case $C=C_{1} \vee C_{2}$, definition of $\mathcal{D}_{\alpha}^{\Rightarrow}(C)$.

Since $\alpha \nVdash C_{1} \vee C_{1}$, we have $\alpha \nVdash C_{1}$ and $\alpha \nVdash C_{2}$. By (IH2), for every $k \in$ $\{1,2\}$ there is an FRJ $(G)$-derivation $\mathcal{D}_{\alpha}\left(C_{k}\right)$ of $\sigma_{\alpha}\left(C_{k}\right)=\Sigma_{k} ; \Theta_{k} \rightarrow C_{k}$ satisfying (Q1) and (Q2). Let $\Sigma_{k}=\Sigma_{k}^{\mathrm{At}} \cup \Sigma_{k}^{\supset}$ and $\Theta_{k}=\Theta_{k}^{\mathrm{At}} \cup \Theta_{k}^{\supset}$ and let

$$
\Upsilon=\left\{Y \mid Y \supset Z \in \Lambda_{\alpha}^{* \supset}\right\} \cup\left\{C_{1}, C_{2}\right\}=\left\{A_{1}, \ldots, A_{n}\right\} \quad(n \geq 1) .
$$

We argue as in the case concerning $\mathcal{D}_{\alpha}^{\Rightarrow}(C)$ with $C \in \mathcal{V}^{\perp}$. For every $1 \leq j \leq n$, since $\alpha \nVdash A_{j}$, by (IH2) there is an $\mathbf{F R J}(G)$-derivation $\mathcal{D}_{\alpha}\left(A_{j}\right)$ of $\sigma_{\alpha}^{\rightarrow}\left(A_{j}\right)=$ $\Sigma_{j} ; \Theta_{j} \rightarrow A_{j}$ such that:

- if $A_{j} \in\left\{C_{1}, C_{2}\right\}$, points (Q1) and (Q2) hold;

- otherwise, points (P1) and (P2) hold.

Hence, we can build the $\mathbf{F R J}(G)$-derivation $\mathcal{D}_{\alpha}^{\Rightarrow}(C)$ as follows $\left(\Sigma^{\mathrm{At}}, \Sigma^{\supset}, \Theta^{\mathrm{At}}\right.$, $\Theta^{\supset}$ are defined as in Fig. 1):

$$
\begin{gathered}
\mathcal{D}_{\alpha}^{\rightarrow}\left(A_{j}\right) \\
\mathcal{D}_{\alpha}^{\Rightarrow}(C)=\frac{\ldots \Sigma_{j}^{\mathrm{At}}, \Sigma_{j}^{\supset} ; \Theta_{j}^{\mathrm{At}}, \Theta_{j}^{\supset} \rightarrow A_{j} \ldots}{\sigma_{\alpha}^{\Rightarrow}(C)=\Gamma \Rightarrow C_{1} \vee C_{2}} \bowtie^{\vee} \quad j=1 \ldots n \\
\Gamma=\Sigma^{\mathrm{At}} \cup \Theta^{\mathrm{At}} \cup \Sigma^{\supset} \cup \Theta^{\supset}
\end{gathered}
$$


We point out that the displayed application of $\bowtie^{\vee}$ matches the restriction (PS4) stated in Sec. 3. Reasoning as above, Point (iii) follows by (P1) and (Q1), Point (iv) (with $\beta=\alpha$ ) by (P2) and (Q2).

- Case $C=C_{1} \wedge C_{2}$, definition of $\mathcal{D}_{\alpha}^{\rightarrow}(C)$.

Since $\alpha \nVdash C_{1} \wedge C_{2}$, there exists $k \in\{1,2\}$ such that $\alpha \nVdash C_{k}$. By (IH3) there exists an $\operatorname{FRJ}(G)$-derivation $\mathcal{D}_{\alpha}\left(C_{k}\right)$ of $\sigma_{\alpha}\left(C_{k}\right)=\Sigma ; \Theta \rightarrow C_{k}$ such that:

(R1) $\operatorname{Rn}\left(\mathcal{D}_{\alpha}^{\rightarrow}\left(C_{k}\right)\right)<\mathrm{h}(\alpha)$;

(R2) $\Sigma \subseteq \Lambda_{\alpha}^{*} \subseteq \Sigma \cup \Theta$.

We can build the $\mathbf{F R J}(G)$-derivation:

$$
\begin{aligned}
& \mathcal{D}_{\alpha}^{\rightarrow}\left(C_{k}\right) \\
& \mathcal{D}_{\alpha}^{\rightarrow}(C)=\frac{\Sigma ; \Theta \rightarrow C_{k}}{\sigma_{\alpha}(C)=\Sigma ; \Theta \rightarrow C_{1} \wedge C_{2}} \wedge
\end{aligned}
$$

Since $\operatorname{Rn}\left(\mathcal{D}_{\alpha}^{\rightarrow}(C)\right)=\operatorname{Rn}\left(\mathcal{D}_{\alpha}^{\rightarrow}\left(C_{k}\right)\right)$, by $\left.(\mathrm{R} 1)\right]$ we get $\operatorname{Rn}\left(\mathcal{D}_{\alpha}^{\rightarrow}(C)\right)<\mathrm{h}(\alpha)$, and this proves (i)] Point (ii) immediately follows by (R2)

- Case $C=C_{1} \wedge C_{2}$, definition of $\mathcal{D}_{\alpha}^{\Rightarrow}(C)$.

Since $\alpha \nVdash C_{1} \wedge C_{2}$, there exists $k \in\{1,2\}$ such that $\alpha \nVdash C_{k}$. By (IH2) there exists an $\operatorname{FRJ}(G)$-derivation $\mathcal{D}_{\alpha}^{\Rightarrow}\left(C_{k}\right)$ of $\sigma_{\alpha}^{\Rightarrow}\left(C_{k}\right)=\Gamma \Rightarrow C_{k}$ such that:

(R3) $\operatorname{Rn}\left(\mathcal{D}_{\alpha}^{\Rightarrow}\left(C_{k}\right)\right) \leq \mathrm{h}(\alpha)$;

(R4) There is $\beta \in P$ such that $\alpha \leq \beta$ and $\Lambda_{\beta}^{*} \subseteq \Gamma$.

We can build the $\mathbf{F R J}(G)$-derivation:

$$
\mathcal{D}_{\alpha}^{\Rightarrow}(C)=\frac{\mathcal{D}_{\alpha}^{\Rightarrow}\left(C_{k}\right)}{\Gamma \Rightarrow C_{k}}
$$

Since $\operatorname{Rn}\left(\mathcal{D}_{\alpha}^{\Rightarrow}(C)\right)=\operatorname{Rn}\left(\mathcal{D}_{\alpha}^{\Rightarrow}\left(C_{k}\right)\right)$, by $(\mathrm{R} 3)$ we get $\operatorname{Rn}\left(\mathcal{D}_{\alpha}^{\Rightarrow}(C)\right) \leq \mathrm{h}(\alpha)$, and this proves (iii), Point (iv) immediately follows by (R4).

- Case $C=A \supset B$, definition of $\mathcal{D}_{\alpha}^{\rightarrow}(C)$.

Since $\alpha \nVdash A \supset B$, there is $\eta \in P$ such that $\alpha \leq \eta$ and $\eta \Vdash A$ and $\eta \nVdash B$. Without loss of generality, we assume that, for every $\delta \in P$ such that $\alpha \leq \delta<\eta$, we have $\delta \nVdash A$. Since $\alpha \leq \eta$, it holds that $\alpha \nVdash B$. By (IH3), there exists an $\operatorname{FRJ}(G)$-derivation $\mathcal{D}_{\alpha}^{\rightarrow}(B)$ of $\sigma_{\alpha}^{\rightarrow}(B)=\Sigma_{1} ; \Theta_{1} \rightarrow B$ such that:

(S1) $\operatorname{Rn}\left(\mathcal{D}_{\alpha}^{\rightarrow}(B)\right)<\mathrm{h}(\alpha)$;

(S2) $\Sigma_{1} \subseteq \Lambda_{\alpha}^{*} \subseteq \Sigma_{1} \cup \Theta_{1}$. 
If $\eta=\alpha$, then $\alpha \Vdash A$, hence $A \in \Lambda_{\alpha}$, which implies, by Lemma 15, $A \in \mathcal{C} l\left(\Lambda_{\alpha}^{*}\right)$. Let $\Lambda$ be a (possibly empty) minimum subset of $\Lambda_{\alpha}^{*} \backslash \Sigma_{1}$ such that $A \in \mathcal{C l}\left(\Sigma_{1} \cup \Lambda\right)$ (namely: $\Lambda^{\prime} \subsetneq \Lambda$ implies $A \notin \mathcal{C l}\left(\Sigma_{1} \cup \Lambda^{\prime}\right)$ ); note that $\Lambda \subseteq \Theta_{1}$. We can build the FRJ $(G)$-derivation $\mathcal{D}_{\alpha}(C)$ as follows, where rule $\supset_{\in}$ shifts the set $\Lambda$ to the left of semicolon:

$$
\mathcal{D}_{\alpha}^{\rightarrow}(C)=\begin{array}{cl}
\Sigma_{1} ; \overbrace{\Theta_{2}, \Lambda}^{\mathcal{D}_{\alpha}} \rightarrow B) & \begin{array}{l}
\Theta_{2}=\Theta_{1} \backslash \Lambda \\
A \in \mathcal{C} l\left(\Sigma_{1} \cup \Lambda\right)
\end{array} \\
\frac{\sigma_{\alpha}(C)=\underbrace{\Sigma_{1}, \Lambda}_{\Sigma} ; \Theta_{2} \rightarrow A \supset B}{\Lambda^{\prime} \subsetneq \Lambda \text { implies } A \notin \mathcal{C l}\left(\Sigma_{1} \cup \Lambda^{\prime}\right)} &
\end{array}
$$

We point out that the choice of $\Lambda$ complies with the restriction (PS1) stated in Sec. 3. Since $\Sigma_{1} \subseteq \Lambda_{\alpha}^{*}$ (see (S2)] and $\Lambda \subseteq \Lambda_{\alpha}^{*}$, we get $\Sigma_{1} \cup \Lambda \subseteq \Lambda_{\alpha}^{*}$, namely $\Sigma \subseteq \Lambda_{\alpha}^{*}$. Moreover, since $\Lambda_{\alpha}^{*} \subseteq \Sigma_{1} \cup \Theta_{1}$ (see (S2) and $\Sigma_{1} \cup \Theta_{1}=\Sigma \cup \Theta_{2}$, we get $\Lambda_{\alpha}^{*} \subseteq \Sigma \cup \Theta_{2}$, and this concludes the proof of (ii)] Since $\operatorname{Rn}\left(\mathcal{D}_{\alpha}(C)\right)=$ $\operatorname{Rn}\left(\mathcal{D}_{\alpha} \rightarrow(B)\right)$, by (S1) we get $\operatorname{Rn}\left(\mathcal{D}_{\alpha}(C)\right)<\mathrm{h}(\alpha)$, which proves (i).

If $\alpha<\eta$, then $\mathrm{h}(\eta)<\mathrm{h}(\alpha)$. By the choice of $\eta$, we have $\alpha \nVdash A$. Since $\eta \nVdash B$, by (IH1) there is an $\mathbf{F R J}(G)$-derivation $\mathcal{D}_{\eta}^{\Rightarrow}(B)$ of $\sigma_{\eta}^{\Rightarrow}(B)=\Gamma \Rightarrow B$ such that:

(S3) $\operatorname{Rn}\left(\mathcal{D}_{\eta} \Rightarrow(B)\right) \leq \mathrm{h}(\eta)$;

(S4) There exists $\beta \in P$ such that $\eta \leq \beta$ and $\Lambda_{\beta}^{*} \subseteq \Gamma$.

Since $\eta \Vdash A$ and $\eta \leq \beta$, we get $\beta \Vdash A$, hence $A \in \Lambda_{\beta}$. By Lemma 15, $A \in \mathcal{C l}\left(\Lambda_{\beta}^{*}\right)$ hence, by $(\mathrm{S} 4)]$ and $(\mathcal{C} l 4)] A \in \mathcal{C l}(\Gamma)$. Since $\alpha \nVdash A$, we have $A \notin \Lambda_{\alpha}$ hence, by Lemma $15, A \notin \mathcal{C l}\left(\Lambda_{\alpha}^{*}\right)$. Since $\alpha<\eta \leq \beta$, we have $\Lambda_{\alpha}^{*} \subseteq \Lambda_{\beta}$. By Lemma 15, we get $\Lambda_{\beta}=\mathcal{C} l\left(\Lambda_{\beta}^{*}\right)$, thus $\Lambda_{\alpha}^{*} \subseteq \mathcal{C l}\left(\Lambda_{\beta}^{*}\right)$. By (S4) and $(\mathcal{C} l 4), \mathcal{C l}\left(\Lambda_{\beta}^{*}\right) \subseteq \mathcal{C l}(\Gamma)$, hence $\Lambda_{\alpha}^{*} \subseteq \mathcal{C l}(\Gamma)$. To sum up:

- $\Lambda_{\alpha}^{*} \subseteq \mathcal{C l}(\Gamma) \cap \bar{\Gamma}$ and $A \in \mathcal{C l}(\Gamma) \backslash \mathcal{C l}\left(\Lambda_{\alpha}^{*}\right)$.

Let $\Theta$ be a maximum extension of $\Lambda_{\alpha}^{*}$ such that $\Lambda_{\alpha}^{*} \subseteq \Theta \subseteq \mathcal{C l}(\Gamma) \cap \bar{\Gamma}$ and $A \notin \mathcal{C l}(\Theta)$ (namely: $\Theta \subsetneq \Theta^{\prime} \subseteq \mathcal{C} l(\Gamma) \cap \bar{\Gamma}$ implies $A \in \mathcal{C} l\left(\Theta^{\prime}\right)$ ). We can build the FRJ $(G)$-derivation:

$$
\mathcal{D}_{\alpha}^{\rightarrow}(C)=\begin{array}{cl}
\mathcal{D}_{\eta}^{\Rightarrow}(B) & \Lambda_{\alpha}^{*} \subseteq \Theta \subseteq \mathcal{C l}(\Gamma) \cap \bar{\Gamma} \\
\Gamma \Rightarrow B & A \in \mathcal{C} l(\Gamma) \backslash \mathcal{C l}(\Theta) \\
\hline \sigma_{\alpha}^{\rightarrow}(C)=\cdot ; \Theta \rightarrow A \supset B & \supset \notin \Theta^{\prime} \subseteq \mathcal{C} l(\Gamma) \cap \bar{\Gamma} \text { implies } A \in \mathcal{C l} l\left(\Theta^{\prime}\right)
\end{array}
$$

We point out that the choice of $\Theta$ matches the restriction (PS2) stated in Sec. 3 . We have $\operatorname{Rn}\left(\mathcal{D}_{\alpha}^{\rightarrow}(C)\right)=\operatorname{Rn}\left(\mathcal{D}_{\eta}^{\Rightarrow}(B)\right)$; by (S3) we get $\operatorname{Rn}\left(\mathcal{D}_{\alpha}^{\rightarrow}(C)\right) \leq \mathrm{h}(\eta)$, hence $\operatorname{Rn}\left(\mathcal{D}_{\alpha} \rightarrow(C)\right)<\mathrm{h}(\alpha)$, which proves (i). Since $\Lambda_{\alpha}^{*} \subseteq \Theta$, (ii) holds.

- Case $C=A \supset B$, definition of $\mathcal{D}_{\alpha}^{\Rightarrow}(C)$.

Since $\alpha \nVdash A \supset B$, there is $\eta \in P$ such that $\alpha \leq \eta$ and $\eta \Vdash A$ and $\eta \nVdash B$. Since $\eta \nVdash B$, by induction hypothesis (IH1) if $\alpha<\eta$ and (IH3) if $\alpha=\eta$, there 


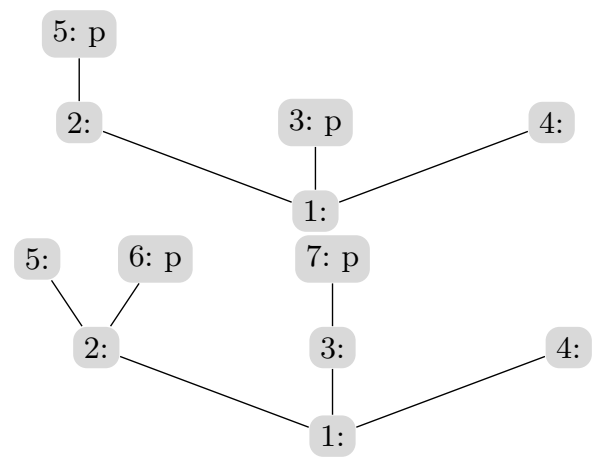

Fig. 10. The countermodels for $S$ and $T$ (see Ex. 5 built by the prover lsj 9 .

is an $\mathbf{F R J}(G)$-derivation $\mathcal{D}_{\eta}^{\Rightarrow}(B)$ of $\sigma_{\eta}^{\Rightarrow}(B)=\Gamma \Rightarrow B$ satisfying points (S3) and (S4) note that $\alpha \leq \eta \leq \beta$. Since $\eta \leq \beta$, we have $\beta \Vdash A$, namely $A \in \Lambda_{\beta}$. By Lemma 15, $A \in \mathcal{C l}\left(\Lambda_{\beta}^{*}\right)$, which implies, by (S4) and $(\mathcal{C} l 4), A \in \mathcal{C l}(\Gamma)$. Thus, we can build the $\mathbf{F R J}(G)$-derivation:

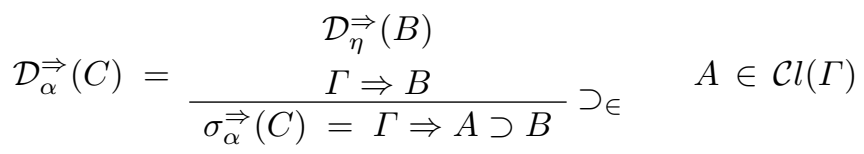

Since $\operatorname{Rn}\left(\mathcal{D}_{\alpha}^{\Rightarrow}(C)\right)=\operatorname{Rn}\left(\mathcal{D}_{\eta}^{\Rightarrow}(B)\right)$ and $\mathrm{h}(\eta) \leq \mathrm{h}(\alpha)$ (indeed, $\left.\alpha \leq \eta\right)$, by (S3) we get $\operatorname{Rn}\left(\mathcal{D}_{\alpha}^{\Rightarrow}(C)\right) \leq \mathrm{h}(\alpha)$, which proves (iii). Point (iv) immediately follows by (S4)] being $\alpha \leq \beta$.

\section{Related and future work}

We have introduced a forward calculus $\mathbf{F R J}(G)$ to derive the non-validity of a goal formula $G$ in IPL. If $G$ is provable in $\operatorname{FRJ}(G)$, from the derivation we can extract a countermodel for $G$. Otherwise, we eventually get a saturated database $\mathrm{D}_{G}$, which can be exploited to build a derivation of $G$ in the sequent calculus G3i; accordingly, $\mathrm{D}_{G}$ can be understood as a "proof-certificate" of the validity of $G$ in IPL (a dual remark for a forward calculus for IPL has been issued in [18]).

To evaluate the potential of our approach, we have developed $f r j$, a Java implementation of our proof-search procedure based on the full-fledged framework JTabWb [11. So far we have implemented the plain forward strategy and the redundancy checks based on forward and backward subsumption. At each iteration of the main loop, frj applies all the possible instances of rules $\wedge, \vee$, $\supset_{\in}$ and $\supset_{\notin}$ involving at least a premise proved in the last step. To manage join rules $\bowtie^{\text {At }}$ and $\bowtie^{\vee}$, frj maintains a list of join-compatible sets $\mathcal{J}_{k}$, namely $\mathcal{J}_{k}$ is a set of irregular sequents matching the side conditions (J1) and (J2) (see Sec. 3). At each iteration the list is updated resting on the set $\mathcal{I}$ of irregular sequents 
proved in the last iteration. In particular, each join-compatible set $\mathcal{J}_{k}$ is possibly extended with elements of $\mathcal{I}$ and the new join-compatible sets issued from $\mathcal{I}$ are added. For every join-compatible set $\mathcal{J}_{k}$, every possible join rule having premises $\mathcal{J}_{k}$ is applied. We also exploit backward subsumption to optimize the implementation of join rules: whenever backward subsumption is detected, every subsumed irregular sequent occurring in a join-compatible set is replaced by the subsuming one. Finally frj, executed with the -gbu option, extracts from the saturated database generated by a failed proof-search in $\mathbf{F R J}(G)$, the $\mathbf{G b u}(G)$ derivation of $G$. When executed with the -latex option, the prover yields the LATEX-rendering of the generated derivations and countermodels. Most of the examples presented in the papers have been obtained by running $f r j$.

As discussed in Sec. 3. whenever we search for an $\mathbf{F R J}(G)$-derivation of $G$, we are also trying to build a countermodel for $G$ in a backward style, starting from the final worlds down to the root. Thus, our countermodel construction technique is dual to standard proof-search procedures such as [18|9|10|13|19|20, where proofs and model are searched bottom-up, starting from the goal and backward applying the rules of the calculus. One of the advantages of forward vs. backward reasoning is that, provided one implements suitable redundancy checks, derivations are more concise since sequents are reused and not duplicated. As a consequence, the obtained models are in general compact and do not contain redundant worlds. For instance, the models in Figs. 5 and 6 are the minimal countermodels for the formulas $S$ and $T$ respectively. The model in Fig. 6 is particularly significant since it is not a tree, hence it cannot be obtained by the mentioned standard proof-search procedures, which only generate tree-shaped models. For instance, let us consider the prover $1 \mathbf{s j}$, implementing in JTabWb a backward proof-search procedure for the calculi presented in [9. For unprovable formulas, $1 \mathrm{sj}$ yields countermodels of minimal height; however, the obtained models are always trees, hence they might contain redundant worlds. In Fig. 10 we show the countermodels built by $1 \mathrm{sj}$ for the formulas $S$ and $T$; in the lefthand side model, the worlds 3 and 5 can be overlapped; in the right-hand side model, world 6 can be merged with 7 and 4 with 5 . As another significant example, let us consider the one-variable formulas $N_{i}$ of the Nishimura family [3], which are not valid in IPL:

$$
\begin{array}{ll}
N_{1}=p & N_{2 n+3}=N_{2 n+1} \vee N_{2 n+2} \\
N_{2}=\neg p & N_{2 n+4}=N_{2 n+3} \supset N_{2 n+1} \quad n \geq 0
\end{array}
$$

We recall that the aforementioned formulas $S$ and $T$ are equivalent to $N_{10}$ and $N_{9}$ respectively. For formulas $N_{j}$, frj generates the standard "tower-like" minimum countermodel [3. In Fig. 11] we display the countermodel obtained for $N_{17}$; in contrast, 1 s j fails to build a countermodel for such a formula.

A method to test the validity of intuitionistic formulas, based on the construction of small countermodels, is presented in $14 / 22$. Let $G$ be the goal formula and let us call atoms the subformulas of $G$ which are propositional variables or $\supset$-formulas (note that these are the same kind of formulas handled by $\mathbf{F R J}(G)$ ). The construction of a countermodel for $G$ is based on a fixpoint procedure. The 


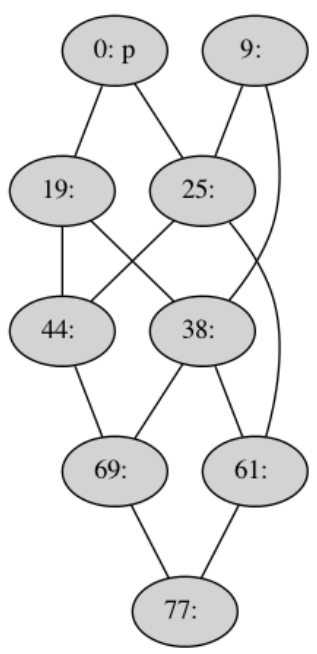

Fig. 11. Countermodel for $N_{17}$

initial configuration is the structure $\mathcal{K}_{0}=\left\langle P_{0}, \leq, w_{0}, V\right\rangle$, where $P_{0}$ is the power set of the set of atoms, $\leq$ is the subset relation, $w_{0}$ is the empty set (hence, $\left\langle P_{0}, \leq, w_{0}\right\rangle$ is a poset with minimum element $w_{0}$ ) and $V$ is a function mapping $w \in P_{0}$ to the set $w \cap \mathcal{V}$. By iterating a refinement procedure, consisting in removing elements from $P_{0}$, we eventually get a Kripke model $\mathcal{K}_{n}=\left\langle P_{n}, \leq, w_{n}, V\right\rangle$ satisfying the following properties:

- for every $w \in P_{n}$ and every atom $A, \mathcal{K}_{n}, w \Vdash A$ iff $A \in w$;

- for every $w \in P_{n}$ and every subformula $F$ of $G, \mathcal{K}_{n}, w \Vdash F$ iff $F \in \mathcal{C l}(w)$;

- $G$ is not valid in IPL iff $\mathcal{K}_{n}$ is a countermodel for $G$ (namely, $\mathcal{K}_{n}, w_{n} \nVdash G$ ).

Thus, to decide the validity of $G$, one has to run the process until the fixpoint $\mathcal{K}_{n}$ is reached, and then check whether $\mathcal{K}_{n}$ is a countermodel for $G$ (e.g., by testing whether $\left.G \in \mathcal{C l}\left(w_{n}\right)\right)$. The procedure has been implemented by the system BDDIntKt, using BDD (Binary Decision Diagram) to efficiently represent the posets and the operations on them. By construction, the generated countermodels do not contain redundancies (indeed, distinct worlds of $\mathcal{K}_{n}$ are separated by at least one atom). However, the properties of the obtained countermodels are not fully investigated in the mentioned papers and BDDIntKt does not explicitly output them; we guess that they are close to the ones obtained with $f r j$. We also point out that 22] presents a procedure (not implemented in BDDIntKt) to get a derivation of $G$ in a standard sequent calculus in case the final model $\mathcal{K}_{n}$ is not a countermodel for $G$.

Finally, as a future work we plan to investigate the applicability of our method to other logics, in particular to modal logics such as $\mathbf{S} \mathbf{4}$ and intermediate logics such as Gödel-Dummett logic characterized by linear Kripke models. 


\section{References}

1. A. Avellone, C. Fiorentini, and A. Momigliano. A semantical analysis of focusing and contraction in intuitionistic logic. Fundamenta Informaticae, 140(3-4):247-262, 2015.

2. T. Brock-Nannestad and K. Chaudhuri. Disproving using the inverse method by iterative refinement of finite approximations. In H. De Nivelle, editor, TABLEAUX 2015, volume 9323 of $L N C S$, pages 153-168. Springer, 2015.

3. A. Chagrov and M. Zakharyaschev. Modal Logic. Oxford University Press, 1997.

4. K. Chaudhuri and F. Pfenning. A focusing inverse method theorem prover for firstorder linear logic. In R. Nieuwenhuis, editor, CADE-20, volume 3632 of LNCS, pages 69-83. Springer, 2005.

5. K. Chaudhuri, F. Pfenning, and G. Price. A logical characterization of forward and backward chaining in the inverse method. In U. Furbach et al., editor, IJCAR 2006, volume 4130 of $L N C S$, pages 97-111. Springer, 2006.

6. A. Degtyarev and A. Voronkov. The inverse method. In J.A. Robinson et al., editor, Handbook of Automated Reasoning, pages 179-272. Elsevier and MIT Press, 2001.

7. K. Donnelly, T. Gibson, N. Krishnaswami, S. Magill, and S. Park. The inverse method for the logic of bunched implications. In F. Baader et al., editor, LPAR 2004, volume 3452 of $L N C S$, pages 466-480. Springer, 2004.

8. M. Ferrari, C. Fiorentini, and G. Fiorino. FCube: An efficient prover for intuitionistic propositional logic. In C. G. Fermüller et al., editor, LPAR 2010, volume 6397 of $L N C S$, pages 294-301. Springer, 2010.

9. M. Ferrari, C. Fiorentini, and G. Fiorino. Contraction-free linear depth sequent calculi for intuitionistic propositional logic with the subformula property and minimal depth counter-models. Journal of Automated Reasoning, 51(2):129-149, 2013.

10. M. Ferrari, C. Fiorentini, and G. Fiorino. An evaluation-driven decision procedure for G3i. ACM Transactions on Computational Logic (TOCL), 16(1):8:1-8:37, 2015.

11. M. Ferrari, C. Fiorentini, and G. Fiorino. JTabWb: a Java framework for implementing terminating sequent and tableau calculi. Fundamenta Informaticae, 150:119-142, 2017.

12. C. Fiorentini and M. Ferrari. A forward unprovability calculus for intuitionistic propositional logic. In R. A. Schmidt and C. Nalon, editors, TABLEAUX 2017, volume 10501 of $L N C S$, pages 114-130. Springer, 2017.

13. R. Goré and L. Postniece. Combining derivations and refutations for cut-free completeness in bi-intuitionistic logic. Journal of Logic and Computation, 20(1):233260, 2010.

14. R. Goré and J. D. Monk Thomson. Bdd-based automated reasoning for propositional bi-intuitionistic tense logics. In Bernhard Gramlich, Dale Miller, and Uli Sattler, editors, Automated Reasoning - 6th International Joint Conference, IJCAR 2012, Manchester, UK, June 26-29, 2012. Proceedings, volume 7364, pages 301-315. Springer, 2012.

15. L. Kovács, A. Mantsivoda, and A. Voronkov. The inverse method for many-valued logics. In F. Castro-Espinoza et al., editor, MICAI 2013, volume 8265 of LNCS, pages 12-23. Springer, 2013.

16. V. Lifschitz. What is the inverse method? J. Automat. Reason., 5(1):1-23, 1989.

17. S. Ju. Maslov. An invertible sequential version of the constructive predicate calculus. Zap. Naučn. Sem. Leningrad. Otdel. Mat. Inst. Steklov. (LOMI), 4:96-111, 1967. 
18. S. McLaughlin and F. Pfenning. Imogen: Focusing the polarized inverse method for intuitionistic propositional logic. In I. Cervesato et al., editor, LPAR 2008, volume 5330 of $L N C S$, pages 174-181. Springer, 2008.

19. S. Negri. Proofs and countermodels in non-classical logics. Logica Universalis, $8(1): 25-60,2014$.

20. L. Pinto and R. Dyckhoff. Loop-free construction of counter-models for intuitionistic propositional logic. In Behara et al., editor, Symposia Gaussiana, Conference A, pages 225-232. Walter de Gruyter, Berlin, 1995.

21. T. Skura. A complete syntactical characterization of the intuitionistic logic. Reports on Mathematical Logic, 75(8):75-80, 1989.

22. J. Thomson. Using BDDs for Non-Classical Propositional Theorem Proving. PhD thesis, The Australian National University, February 2014.

23. A.S. Troelstra and H. Schwichtenberg. Basic Proof Theory, volume 43 of Cambridge Tracts in Theoretical Computer Science. Camb. Univ. Press, 2ed edition, 2000. 


\section{A Soundness of $\operatorname{FRJ}(G)$}

We prove that, given an $\mathbf{F R J}(G)$-derivation $\mathcal{D}$ of $G, \operatorname{Mod}(\mathcal{D})$ is a countermodel for $G$. As discussed in Sec. 3, we have to prove the soundness property (S1) We introduce the following relation $\mapsto_{*}^{\mathrm{Ir}}$ between irregular sequents:

$-\sigma_{1} \mapsto_{*}^{\operatorname{Ir}} \sigma_{2}$ iff $\sigma_{1} \mapsto_{*} \sigma_{2}$ and every $\sigma^{\prime}$ such that $\sigma_{1} \mapsto_{*} \sigma^{\prime} \mapsto_{*} \sigma_{2}$ is irregular.

It is easy to check that:

(Ir1) $\Sigma_{1} ; \Theta_{1} \rightarrow C_{1} \mapsto_{*}^{\operatorname{Ir}} \Sigma_{2} ; \Theta_{2} \rightarrow C_{2}$ implies $\Sigma_{1} \subseteq \Sigma_{2}$ and $\Sigma_{2} \cup \Theta_{2} \subseteq \Sigma_{1} \cup \Theta_{1}$.

The key lemma is (we recall that $\mathrm{Sf}^{-}(C)$ denotes the set $\operatorname{Sf}(C) \backslash\{C\}$ ):

Lemma 16 (aka Lemma 3.9 of Sec.3). Let $\mathcal{D}$ be an $\mathbf{F R J}(G)$-derivation of $G$, let $\operatorname{Mod}(\mathcal{D})$ be the model extracted from $\mathcal{D}$ and $\phi$ the map associated with $\mathcal{D}$. For every sequent $\sigma$ occurring in $\mathcal{D}$ :

(i) if $\sigma=\Gamma \Rightarrow C$, then $\phi(\sigma) \Vdash \Gamma$ and $\phi(\sigma) \nVdash C$;

(ii) if $\sigma=\Sigma ; \Theta \rightarrow C$, let $\sigma_{p} \in \mathrm{P}(\mathcal{D})$ such that $\sigma \mapsto \sigma_{p}$ and $\sigma_{p} \Vdash \Sigma \cap \mathrm{Sf}^{-}(C)$; then $\sigma_{p} \nVdash C$.

Proof. We prove the assertions by a main induction (IH1) on the height $\mathrm{h}(\sigma)$ of $\sigma$ in $\mathcal{D}$. Let $\operatorname{Mod}(\mathcal{D})=\langle\mathrm{P}(\mathcal{D}), \leq, \rho, V\rangle$ and let $\mathcal{R}$ be the rule applied to get $\sigma$; we proceed by a case analysis on $\mathcal{R}$. In discussing the cases, we use the notation in Fig. 1. We also recall that

$$
\bar{\Gamma}^{\mathrm{At}}=\mathrm{SL}(G) \cap \mathcal{V} \quad \bar{\Gamma}^{\supset}=\mathrm{SL}(G) \cap \mathcal{L}^{\supset} \quad \bar{\Gamma}=\bar{\Gamma}^{\mathrm{At}} \cup \bar{\Gamma}^{\supset}
$$

$-\mathcal{R}=\mathrm{Ax}_{\Rightarrow}$

We have $\sigma=\bar{\Gamma}^{\text {At }} \backslash\{C\} \Rightarrow C$, with $C \in \mathcal{V}^{\perp}$. Since $\phi(\sigma)=\sigma$ and $V(\sigma)=$ $\bar{\Gamma}^{\text {At }} \backslash\{C\}$, (i) immediately follows.

$-\mathcal{R}=\mathrm{Ax}_{\rightarrow}$.

We have $\sigma=\cdot ; \bar{\Gamma}^{\text {At }} \backslash\{C\}, \bar{\Gamma}^{\supset} \rightarrow C$, with $C \in \mathcal{V}^{\perp}$. Let $\Gamma^{\text {At }}=V\left(\sigma_{p}\right)$. Since $\sigma \mapsto \sigma_{p}$, by Lemma 1 (iii) and $(\mathcal{C} l 5)$ we get $\Gamma^{\mathrm{At}} \subseteq \bar{\Gamma}^{\mathrm{At}} \backslash\{C\}$. This implies $C \notin \Gamma^{\mathrm{At}}$, hence $\sigma_{p} \nVdash C$, which proves (ii).

$-\mathcal{R}=\wedge$.

Both (i) and (ii) easily follow by (IH1).

$-\mathcal{R}=\vee$. 
We have

$$
\frac{\sigma_{1}=\Sigma_{1} ; \Theta_{1} \rightarrow C_{1} \quad \sigma_{2}=\Sigma_{2} ; \Theta_{2} \rightarrow C_{2}}{\sigma=\underbrace{\Sigma_{1}, \Sigma_{2}}_{\Sigma} ; \underbrace{\Theta_{1} \cap \Theta_{2}}_{\Theta} \rightarrow C_{1} \vee C_{2}} \vee \begin{aligned}
& \Sigma_{1} \subseteq \Sigma_{2} \cup \Theta_{2} \\
& \Sigma_{2} \subseteq \Sigma_{1} \cup \Theta_{1}
\end{aligned}
$$

By hypothesis, $\sigma \mapsto \sigma_{p}$ and $\sigma_{p} \Vdash \Sigma \cap \mathrm{Sf}^{-}\left(C_{1} \vee C_{2}\right)$. Let $k \in\{1,2\}$. We have both $\sigma_{k} \mapsto \sigma_{p}$ (indeed, $\sigma_{k} \mapsto_{0} \sigma$ and $\left.\sigma \mapsto \sigma_{p}\right)$ and $\sigma_{p} \Vdash \Sigma_{k} \cap \mathrm{Sf}^{-}\left(C_{k}\right)$ (indeed, $\Sigma_{k} \subseteq \Sigma$ and $\left.\mathrm{Sf}^{-}\left(C_{k}\right) \subseteq \mathrm{Sf}^{-}\left(C_{1} \vee C_{2}\right)\right)$. By (IH1) applied to $\sigma_{k}$, we get $\sigma_{p} \nVdash C_{k}$. Thus, both $\sigma_{p} \nVdash C_{1}$ and $\sigma_{p} \nVdash C_{2}$, hence $\sigma_{p} \nVdash C_{1} \vee C_{2}$ which proves (ii).

$-\mathcal{R}=\supset_{\epsilon}$.

If $\sigma$ is regular, we have

$$
\frac{\sigma_{1}=\Gamma \Rightarrow B}{\sigma=\Gamma \Rightarrow A \supset B} \supset \in \quad A \in \mathcal{C l}(\Gamma)
$$

By (IH1) applied to $\sigma_{1}$, it holds that $\phi\left(\sigma_{1}\right) \Vdash \Gamma$ and $\phi\left(\sigma_{1}\right) \nVdash B$. Since $A \in \mathcal{C l}(\Gamma)$, by $(\mathcal{C} l 1)$ we get $\phi\left(\sigma_{1}\right) \Vdash A$. Since $\phi(\sigma)=\phi\left(\sigma_{1}\right)$, we get $\phi(\sigma) \nVdash A \supset B$, which implies (i).

Let $\sigma$ be irregular and let us assume:

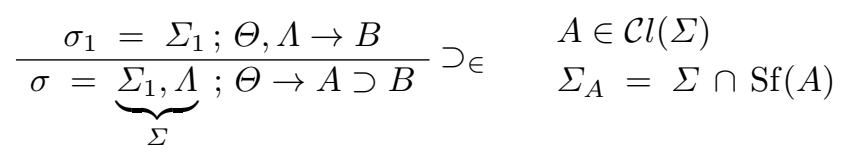

By hypothesis $\sigma \mapsto \sigma_{p}$ and $\sigma_{p} \Vdash \Sigma \cap \mathrm{Sf}^{-}(A \supset B)$. Thus, $\sigma_{p} \Vdash \Sigma_{1} \cap \mathrm{Sf}^{-}(B)$ and, since $\operatorname{Sf}(A) \subseteq \operatorname{Sf}^{-}(A \supset B)$, we get $\sigma_{p} \Vdash \Sigma_{A}$. By hypothesis $\sigma \mapsto \sigma_{p}$, hence $\sigma_{1} \mapsto \sigma_{p}$; we can apply (IH1) to $\sigma_{1}$ and infer $\sigma_{p} \nVdash B$. Since $A \in \mathcal{C} l(\Sigma)$, by $(\mathcal{C} l 2)$ we get $A \in \mathcal{C l}\left(\Sigma_{A}\right)$. By the fact that $\sigma_{p} \Vdash \Sigma_{A}$ and $(\mathcal{C} l 1)$, we get $\sigma_{p} \Vdash A$. We conclude $\sigma_{p} \nVdash A \supset B$, which proves (ii).

$-\mathcal{R}=\supset_{\sharp}$.

We have:

$$
\frac{\sigma_{1}=\Gamma \Rightarrow B}{\sigma=\cdot ; \Theta \rightarrow A \supset B} \supset_{\notin} \quad A \in \mathcal{C l}(\Gamma)
$$

By (IH1) applied to $\sigma_{1}$, we have $\phi\left(\sigma_{1}\right) \Vdash \Gamma$ and $\phi\left(\sigma_{1}\right) \nVdash B$. By $(\mathcal{C} l 1) \phi\left(\sigma_{1}\right) \Vdash A$. By hypothesis $\sigma \mapsto \sigma_{p}$, which implies $\sigma_{1} \mapsto \sigma_{p}$. Thus $\sigma_{p} \leq \phi\left(\sigma_{1}\right)$, hence $\sigma_{p} \nVdash$ $A \supset B$, and this proves (ii).

$-\mathcal{R}=\bowtie^{\mathrm{At}}$.

We have:

$$
\begin{aligned}
& \frac{\ldots \sigma_{j}=\Sigma_{j}^{\mathrm{At}}, \Sigma_{j}^{\supset} ; \Theta_{j}^{\mathrm{At}}, \Theta_{j}^{\supset} \rightarrow A_{j} \ldots}{\sigma=\Sigma^{\mathrm{At}}, \Theta^{\mathrm{At}} \backslash\{C\}, \Sigma^{\supset}, \Theta^{\supset} \Rightarrow C} \bowtie^{\mathrm{At}} \quad \begin{array}{l}
j=1 \ldots n \\
C \in \mathcal{V}^{\perp} \backslash \Sigma^{\mathrm{At}}
\end{array} \\
& \Gamma^{\mathrm{At}}=\Sigma^{\mathrm{At}} \cup\left(\Theta^{\mathrm{At}} \backslash\{C\}\right) \quad \Gamma^{\supset}=\Sigma^{\supset} \cup \Theta^{\supset} \quad \Gamma=\Gamma^{\mathrm{At}} \cup \Gamma^{\supset}
\end{aligned}
$$

Note that $\sigma \in \mathrm{P}(\mathcal{D}), \phi(\sigma)=\sigma$ and $V(\sigma)=\Gamma^{\mathrm{At}}$. Since $C \notin \Gamma^{\mathrm{At}}$, we get: 
(P1) $\sigma \Vdash \Gamma^{\mathrm{At}}$ and $\sigma \nVdash C$.

To complete the proof of (i), it remains to show that $\sigma \Vdash \Gamma^{\supset}$. To this aim, we show that, for every formula $H$, the following properties hold:

(P2) $H \in \Gamma^{\supset}$ implies $\sigma \Vdash H$.

(P3) $H=A_{j}$, with $1 \leq j \leq n$, implies $\sigma \nVdash H$.

To prove (P2) and (P3), we introduce a secondary induction hypothesis (IH2) on $|H|$. Let $H \in \Gamma^{\supset}$. Then, there is $k \in\{1, \ldots, n\}$ such that $H=A_{k} \supset B$. Let $\sigma_{p} \in \mathrm{P}(\mathcal{D})$ such that $\sigma \leq \sigma_{p}$ and $\sigma_{p} \Vdash A_{k}$; we show that $\sigma_{p} \Vdash B$. Let $\Gamma_{p}=\operatorname{Lhs}\left(\sigma_{p}\right)$; since $H \in \operatorname{Lhs}(\sigma)$ and $\sigma_{p} \mapsto_{*} \sigma$ (indeed, $\left.\sigma \leq \sigma_{p}\right)$, by Lemma 1(iii) we get $H \in \mathcal{C l}\left(\Gamma_{p}\right)$. Since $\left|A_{k}\right|<|H|$, we can apply (IH2) on (P3) and claim that $\sigma \nVdash A_{k}$, hence $\sigma_{p} \neq \sigma$, which implies $\mathrm{h}\left(\sigma_{p}\right)<\mathrm{h}(\sigma)$. By (IH1) applied to $\sigma_{p}$, we have $\sigma_{p} \Vdash \Gamma_{p}$ and, by $(\mathcal{C} l 1), \sigma_{p} \Vdash H$, namely $\sigma_{p} \Vdash A_{k} \supset B$. Since $\sigma_{p} \Vdash A_{k}$, we get $\sigma_{p} \Vdash B$; this concludes the proof of (P2).

Let $H=A_{j}$, where $j \in\{1, \ldots, n\}$. Note that $\mathrm{h}\left(\sigma_{j}\right)<\mathrm{h}(\sigma), \sigma_{j} \mapsto_{0} \sigma$ and $\sigma \in \mathrm{P}(\mathcal{D})$. We prove $\sigma \nVdash A_{j}$ by applying (IH1) on $\sigma_{j}$ (Point (ii)]. To this aim, we have to check that the condition

(†) $\sigma \Vdash\left(\Sigma_{j}^{\mathrm{At}} \cup \Sigma_{j}^{\supset}\right) \cap \mathrm{Sf}^{-}\left(A_{j}\right)$

holds. Let $K \in\left(\Sigma_{j}^{\mathrm{At}} \cup \Sigma_{j}^{\supset}\right) \cap \mathrm{Sf}^{-}\left(A_{j}\right)$. If $K \in \Sigma_{j}^{\mathrm{At}}$, since $\Sigma_{j}^{\mathrm{At}} \subseteq V(\sigma)$ we immediately get $\sigma \Vdash K$. If $K \in \Sigma_{j}^{\supset}$, then $K \in \Gamma^{\supset}$ and $|K|<\left|A_{j}\right|$ (indeed, by definition of $\mathrm{Sf}^{-}\left(A_{j}\right), K$ is a proper subformula of $A_{j}$ ). We can apply (IH2) on (P2) and we get $\sigma \Vdash K$, hence $(\dagger)$ holds. This concludes the proof of (P3). By (P1) and (P2), Point (i) follows.

$-\mathcal{R}=\bowtie^{\vee}$.

Similar to the case $\bowtie^{\mathrm{At}}$.

By Lemma 16, we get:

Lemma 17. Soundness properties (S1) and (S2) hold.

Proof. Let $\sigma=\Gamma \Rightarrow C$ be provable in $\operatorname{FRJ}(G)$ and let $\mathcal{D}$ be an $\mathbf{F R J}(G)$ derivation of $\sigma$. By Lemma 16(i) the world $\phi(\sigma)$ of the $\operatorname{model} \operatorname{Mod}(\mathcal{D})$ satisfies $\phi(\sigma) \Vdash \Gamma$ and $\phi(\sigma) \nVdash C$. Setting $\mathcal{K}=\operatorname{Mod}(\mathcal{D})$ and $\alpha=\phi(\sigma)$, (S1) holds.

We show (S2). Let $\sigma=\Sigma ; \Theta \rightarrow C$ be provable in $\mathbf{F R J}(G)$ and let us assume that $\sigma$ can be used to prove a regular sequent $\sigma_{r}$ in $\mathbf{F R J}(G)$. Then, there exist an irregular sequent $\sigma^{\prime}$ and a p-sequent $\sigma_{p}$ such that:

$\sigma=\Sigma ; \Theta \rightarrow C \quad \mapsto_{*}^{\operatorname{Ir}} \quad \sigma^{\prime}=\Sigma^{\prime} ; \Theta^{\prime} \rightarrow C^{\prime} \quad \mapsto_{0} \quad \sigma_{p}=\Gamma_{p} \Rightarrow C_{p} \quad \mapsto_{*} \quad \sigma_{r}$ where $\bowtie$ denotes one between the rules $\bowtie^{\text {At }}$ and $\bowtie^{\vee}$. By Point (Ir1) we have $\Sigma \subseteq \Sigma^{\prime}$ and $\Sigma^{\prime} \cup \Theta^{\prime} \subseteq \Sigma \cup \Theta$. By definition of $\bowtie$, we also have $\Sigma^{\prime} \subseteq \Gamma_{p} \subseteq \Sigma^{\prime} \cup \Theta^{\prime}$; hence $\Sigma \subseteq \Gamma_{p} \subseteq \Sigma \cup \Theta$. Let $\mathcal{D}$ be the $\mathbf{F R J}(G)$-derivation having root sequent $\sigma_{p}$. By Lemma 16|(i) $\sigma_{p} \Vdash \Gamma_{p}$; since $\Sigma \cap \mathrm{Sf}^{-}(C) \subseteq \Gamma_{p}$, we get $\sigma_{p} \Vdash \Sigma \cap \mathrm{Sf}^{-}(C)$. Since $\sigma \mapsto \sigma_{p}$, we can apply Lemma 1 d|(ii) to infer $\sigma_{p} \nVdash C$. Setting $\mathcal{K}=\operatorname{Mod}(\mathcal{D})$, $\alpha=\sigma_{p}$ and $\Gamma=\Gamma_{p}$, (S2) holds. 